\title{
A interpolação complexa e a caracterização dos espaços de potências fracionárias associados a operadores acretivos
}

\author{
Marcelo José Dias Nascimento \\ Orientador: Prof. Dr. Alexandre Nolasco de Carvalho \\ Dissertação apresentada ao Instituto de Ciências Matemáticas e de \\ Computação da Universidade de São Paulo, como parte dos requisitos \\ para obtenção do título de Mestre em Ciências na área: Matemática.
}

USP - São Carlos

Janeiro/2003 
Aos meus pais

Ismael e Dirce com amor... 


\section{Agradecimentos $^{1}$}

Agradeço em primeiro lugar a Deus, que durante este período esteve sempre ao meu lado, dando força e saúde.

Agradeço ao Prof. Alexandre pela orientação, sempre disposto a ajudar. Sua dedicação e entusiasmo foi ferramenta de grande motivação para mim.

Que grande importância tem a família em nossas vidas, nosso refúgio e fortaleza. Aos meus pais, Ismael e Dirce agradeço pelo amor e carinho, as minhas irmãs Ioni e Daiane, obrigado pela amizade, e não poderia me esquecer de meus sobrinhos Leonardo, valeu moleque e Isabelli que ainda nem chegou mais já faz parte da família.

Agradeço ainda de forma especial aos amigos que fiz durante esta caminhada, são tantos que não dá para citar nomes. Todos ajudaram de uma forma ou de outra para o desenvolvimento deste trabalho. Minha gratidão também aos professores que contribuiram para minha formação acadêmica.

\footnotetext{
${ }^{1}$ Este trabalho contou com apoio financeiro da CAPES.
} 


\section{Abstract}

In this work, we study the fractional powers spaces associated to acretive operators and the complex interpolation spaces and we show that in Hilbert spaces they are the same. To that end we show that acretive operators $A: D(A) \subset E \longrightarrow E$ in Hilbert spaces have bounded imaginary powers and that the fractional powers spaces $E^{\alpha}$ associated to operators with bounded imaginary powers coincide with the complex interpolation spaces $\left[E, E^{1}\right]_{\alpha}$. 


\section{Resumo}

Neste trabalho, estudamos os espaços de potências fracionárias associados a operadores acretivos e os espaços de interpolação complexa e mostramos que em espaços de Hilbert estes espaços coincidem. Para este fim mostramos que operadores acretivos $A: D(A) \subset E \longrightarrow E$ em espaços de Hilbert tem potências imaginárias limitadas e que os espaços de potências fracionárias $E^{\alpha}$ associados ao operador com potências imaginárias limitadas coincidem com o espaços de interpolação complexa $\left[E, E^{1}\right]_{\alpha}$. 


\section{Sumário}

Introdução

$\begin{array}{llr}1 & \text { Preliminares } & 7\end{array}$

1.1 Semigrupos e Seus Geradores . . . . . . . . . . . . . . . . . . 7

1.2 O Teorema de Hille-Yosida . . . . . . . . . . . . . . . . . . 9

1.3 O Teorema de Lumer-Phillips . . . . . . . . . . . . . . . . . . . 10

1.4 Pseudo-Resolventes . . . . . . . . . . . . . . . . . . . 13

1.5 O Semigrupo Dual e o Teorema de Stone . . . . . . . . . . . . . . . 16

1.6 Transformada Inversa de Laplace . . . . . . . . . . . . . . . . . . . . . . . 19

1.7 Operadores Setoriais e Analiticidade . . . . . . . . . . . . . . . . . . 21

2 Potências Fracionárias $\quad 25$

2.1 Introdução . . . . . . . . . . . . . . . . . . . . . . . 25

2.2 Operadores do Tipo Positivo f . . . . . . . . . . . . . . . 28

2.3 Potências de Potências Fracionárias . . . . . . . . . . . . . . . 39

2.4 Potências Imaginárias Limitadas $\ldots \ldots$. . . . . . . . . . . . . 46

3 Potências Fracionárias de Operadores Acretivos $\quad 49$

3.1 Potências Fracionárias ． . . . . . . . . . . . . . . . . . . 49

4 Métodos de Interpolação Complexos $\quad 63$

4.1 Introdução . . . . . . . . . . . . . . . . . . . . . . . . 63

4.2 Funtores de Interpolação . . . . . . . . . . . . . . . . . . . . . . . . . 65

4.3 Métodos Complexos de Interpolação ～. . . . . . . . . . . . . . . . . . 67

4.4 Os Espaços $\left[A_{0}, A_{1}\right]_{\theta, \gamma}$ e $\left[A_{0}, A_{1}\right]_{\theta, \gamma,-} \ldots \ldots \ldots \ldots \ldots \ldots$

$\begin{array}{llr}5 & \text { Resultados Finais } & 81\end{array}$

5.1 Potências Fracionárias e Espaços de Interpolação . . . . . . . . . . . . . . 81 
Bibliografia

Índice Remissivo 


\section{Introdução}

Antes de descrever o trabalho que será desenvolvido nesta dissertação vamos apresentar uma motivação para a caracterização dos espaços de potências fracionárias, considerando problemas de valor inicial do tipo

$$
\left\{\begin{array}{l}
\dot{e}=A e+f(e), t>0 \\
e(0)=e_{0}
\end{array}\right.
$$

onde o operador linear $-A: D(A) \subset E^{0} \rightarrow E^{0}$ é um operador setorial em um espaço de Banach $E$. Denotaremos por $E^{\alpha}, \alpha \geq 0$ os espaços de potência fracionária associados ao operador $A$ (veja $[2,3,4,13]$ ) e por $\mathbf{e}^{A t}$ o semigrupo analítico gerado por $A$. Assumimos, sem perda de generalidade, que

$$
\begin{aligned}
& \left\|\mathbf{e}^{A t} e\right\|_{E^{\alpha}} \leq M\|e\|_{E^{\alpha}}, \alpha \geq 0 \\
& \left\|\mathbf{e}^{A t} e\right\|_{E^{\alpha}} \leq M t^{-\alpha}\|e\|_{E}, \alpha \geq 0 .
\end{aligned}
$$

É bem conhecido que se $f: E^{1} \rightarrow E^{\alpha}, \alpha>0$, é Lipschitz contínua em subconjuntos limitados de $E^{1}$ então o problema (1) é localmente bem posto em $E^{1}$. Neste caso, para cada $e_{0} \in E^{1}$ procuramos por pontos fixos de $T$ no espaço $K(\tau, \mu)=\left\{e(t) \in C\left([0, \tau], E^{1}\right) ; e(0)=\right.$ $\left.e_{0},\|e(t)\|_{L^{\infty}\left(0, \tau, E^{1}\right)} \leq\left\|e_{0}\right\|_{E^{1}}+\mu\right\}$, onde $T$ é dado por

$$
(T e)(t)=\mathbf{e}^{A t} e_{0}+\int_{0}^{t} \mathbf{e}^{A(t-s)} f(e(s)) d s .
$$

Os cálculos simples,

$$
\begin{aligned}
& \|(T u)(t)-(T v)(t)\|_{E^{1}} \\
& \leq M \int_{0}^{t}(t-s)^{-1+\alpha}\|f(u(s))-f(v(s))\|_{E^{\alpha}} d s \\
& \leq C M \int_{0}^{t}(t-s)^{-1+\alpha}\|u(s)-v(s)\|_{E^{1}} d s \\
& \leq\left(C M \int_{0}^{t}(t-s)^{-1+\alpha} d s\right) \sup _{0 \leq s \leq t}\left\{\|u(s)-v(s)\|_{E^{1}}\right\}
\end{aligned}
$$


e

$$
\begin{aligned}
& \|(T e)(t)\|_{E^{1}} \leq\left\|\mathbf{e}^{A t} e_{0}\right\|_{E^{1}}+M \int_{0}^{t}(t-s)^{-1+\alpha}\|f(e(s))\|_{E^{\alpha}} d s \\
& \leq\left\|\mathbf{e}^{A t} e_{0}\right\|_{E^{1}}+C M \int_{0}^{t}(t-s)^{-1+\alpha} d s\left(1+\sup _{0 \leq s \leq t}\left\{\|e(s)\|_{E^{1}}\right\}\right)
\end{aligned}
$$

juntamente com o fato que $\left\|\mathbf{e}^{A t} e_{0}\right\|_{E^{1}} \stackrel{t \rightarrow 0^{+}}{\longrightarrow}\left\|e_{0}\right\|_{E^{1}}$,

$$
\int_{0}^{t}(t-s)^{-1+\alpha} d s=t^{\alpha} \int_{0}^{1}(1-s)^{-1+\alpha} d s \rightarrow 0
$$

quando $t \rightarrow 0^{+}$, sugerem que para $\mu>0$ fixo podemos escolher $\tau>0$ suficientemente pequeno tal que $T: K(\tau, \mu) \rightarrow K(\tau, \mu)$ e que $T$ é uma contração estrita em $K(\tau, \mu)$. Uma vez que isto é conseguido, o Teorema do Ponto Fixo de Banach se encarrega da existência e unicidade de solução para a equação integral. Com algum esforço extra podemos mostrar que a solução encontrada é uma solução da equação diferencial (1).

$\mathrm{Na}$ análise acima, a convergência da integral imprópria

$$
\int_{0}^{1}(1-s)^{-1+\alpha} d s
$$

que é equivalente ao fato que $\alpha>0$, é essencial e todo o argumento cai por terra quando $\alpha=0$. Em outras palavras, como $A: E^{1} \rightarrow E$, o fato que $f: E^{1} \rightarrow E^{\alpha}$ com $\alpha>0$ significa que as soluções do problema (1) podem ser obtidas como perturbações do problema linear $\dot{e}=A e$.

Vamos comentar a resolubilidade local do problema (1), quando $\alpha=0$.

É claro que se a única hipótese sobre $f$ é que $f: E^{1} \rightarrow E^{0}$ seja localmente Lipschitz, será impossível mostrar que o problema (1) é localmente bem posto. Por exemplo, tomando $f(e)=-2 A e$, que satisfaz $f: E^{1} \rightarrow E^{0}$ e é globalmente Lipschitz, teremos $\dot{e}=A e+f(e)=-A e$, que não é localmente bem posto, em geral (se $A=\Delta$ então $\dot{e}=-A e$ é a equação do calor para traz). Portanto, alguma condição extra deve ser imposta sobre $f$ para garantir a existência das soluções do problema acima.

Para melhor entender a necessidade de caracterização dos espaços de potências fracionárias e as condições que devemos impor sobre as não-linearidades, consideramos o exemplo dado pela equação,

$$
\left\{\begin{array}{l}
u_{t}=\Delta u+u|u|^{\rho-1}, \quad \Omega \\
u=0 \quad \partial \Omega \\
u(0)=u_{0}
\end{array}\right.
$$

onde $\Omega$ é um domínio limitado com fronteira regular em $\mathbb{R}^{3}$ e $\rho>1$.

É sabido que o operador $\Delta$ pode ser visto como um operador ilimitado em $E=H^{-1}(\Omega)$ com domínio $E^{1}=H_{0}^{1}(\Omega)$. Adicionalmente, os espaços de potência fracionária $E^{\alpha}$ satisfazem as 
seguintes imersões,

$$
\begin{array}{ll}
E^{\alpha} \hookrightarrow H^{2 \alpha-1}(\Omega), & \alpha>1 / 2 \\
E^{\frac{1}{2}}=L^{2}(\Omega), & \\
E^{\alpha} \hookleftarrow H^{2 \alpha-1}(\Omega), &
\end{array}
$$

(veja $[4,21])$.

Se $f(u)=u|u|^{\rho-1}$, então com algumas imersões de Sobolev e com (5), podemos mostrar que para $1<\rho \leq 3$, temos o operador de Nemitskii $f: E^{1} \equiv H_{0}^{1}(\Omega) \rightarrow E^{\frac{1}{2}} \equiv L^{2}$; para $4<\rho \leq 5$ temos $f: E^{1} \equiv H_{0}^{1}(\Omega) \rightarrow H^{\frac{3-\rho}{2}} \hookrightarrow E^{\frac{5-\rho}{4}}$. Portanto, para $1<\rho<5, f: E^{1} \rightarrow E^{\alpha}$ para algum $\alpha>0$. Para $\rho=5, f: E^{1} \rightarrow E$ e estamos no caso crítico $\alpha=0$. Mas observe que para $\rho=5$, usando novamente imersões de Sobolev e (5), obtemos que se $\epsilon>0$ é pequeno então $f: E^{1+\epsilon} \rightarrow E^{5 \epsilon}$, enquanto que o operador linear $A: E^{1+\epsilon} \rightarrow E^{\epsilon}$. Isto significa que, embora $A$ e $f$ podem ser considerados de mesma ordem em $E^{1}$, se consideramos um espaço ligeiramente melhor, $E^{1+\epsilon}$, então $f$ regulariza mais que $A$. Adicionalmente, pode ser visto que $f$ satisfaz, $\forall u, v \in E^{1+\epsilon}$,

$$
\begin{aligned}
& \|f(u)-f(v)\|_{E^{5 \epsilon}} \leq c\|u-v\|_{E^{1+\epsilon}}\left(\|u\|_{E^{1+\epsilon}}^{4}+\|v\|_{E^{1+\epsilon}}^{4}+1\right), \\
& \|f(u)\|_{E^{5 \epsilon}} \leq c\|u\|_{E^{1+\epsilon}}^{5} .
\end{aligned}
$$

Em particular, isto significa que podemos resolver o problema (4) com dado inicial em $E^{1+\epsilon}$. Adicionalmente, se consideramos agora a seqüência de dados iniciais $u_{n} \in E^{1+\epsilon} \operatorname{com} u_{n} \rightarrow u_{0} \in$ $E^{1}$ em $E^{1}$, com os cálculos seguintes,

$$
\begin{aligned}
& t^{\epsilon}\left\|u_{n}(t)\right\|_{E^{1+\epsilon}} \leq t^{\epsilon}\left\|\mathbf{e}^{A t} u_{n}\right\|_{E^{1+\epsilon}}+t^{\epsilon} \int_{0}^{t}(t-s)^{-1+4 \epsilon}\left\|u_{n}(s)^{5}\right\|_{E^{5 \epsilon}} d s \\
& \leq t^{\epsilon}\left\|\mathbf{e}^{A t} u_{n}\right\|_{E^{1+\epsilon}}+t^{\epsilon} \int_{0}^{t}(t-s)^{-1+4 \epsilon} s^{-5 \epsilon} d s \sup _{0<s<t}\left\{s^{\epsilon}\left\|u_{n}(s)\right\|_{E^{1+\epsilon}}\right\}^{5}
\end{aligned}
$$

e o fato que $t^{\epsilon}\left\|\mathbf{e}^{A t} u_{n}\right\|_{E^{1+\epsilon}} \stackrel{t \rightarrow 0^{+}}{\longrightarrow} 0$ uniformemente em compactos de $E^{1}$, não é difícil ver que se $\mu>0$ é suficientemente pequeno, podemos obter um tempo $\tau_{1}>0$, independente de $n$ tal que $t^{\epsilon}\left\|u_{n}(t)\right\|_{E^{1+\epsilon}} \leq \mu$ para todo $t \in\left(0, \tau_{1}\right]$ e todo $n$.

Mas também, para $0<t \leq \tau_{1}$, temos

$$
\begin{aligned}
& t^{\epsilon}\left\|u_{n}(t)-u_{m}(t)\right\|_{E^{1+\epsilon}} \leq t^{\epsilon}\left\|\mathbf{e}^{A t}\left(u_{n}-u_{m}\right)\right\|_{E^{1+\epsilon}} \\
& +t^{\epsilon} \int_{0}^{t}(t-s)^{-1+4 \epsilon}\left\|u_{n}(s)-u_{m}(s)\right\|_{E^{1+\epsilon}}\left(1+\left\|u_{n}(s)\right\|_{E^{1+\epsilon}}^{4}+\left\|u_{m}(s)\right\|_{E^{1+\epsilon}}^{4}\right) d s \\
& \leq\left\|u_{n}-v_{n}\right\|_{E^{1}}+\left(t^{\epsilon} \int_{0}^{t}(t-s)^{-1+4 \epsilon} s^{-\epsilon} d s\right) \sup _{0<s \leq t}\left\{s^{\epsilon}\left\|u_{n}(s)-u_{m}(s)\right\|_{E^{1+\epsilon}}\right\} \\
& +\left(2 \mu^{4} t^{\epsilon} \int_{0}^{t}(t-s)^{-1+4 \epsilon} s^{-5 \epsilon} d s\right) \sup _{0<s \leq t}\left\{s^{\epsilon}\left\|u_{n}(s)-u_{m}(s)\right\|_{E^{1+\epsilon}}\right\}
\end{aligned}
$$


o que implica que para algum $0<t \leq \tau_{0} \leq \tau_{1}$ obtemos

$$
t^{\epsilon}\left\|u_{n}(t)-u_{m}(t)\right\|_{E^{1+\epsilon}} \leq C\left\|u_{n}-u_{m}\right\|_{E^{1}} .
$$

Um argumento semelhante mostra que

$$
\begin{aligned}
\left\|u_{n}(t)-u_{m}(t)\right\|_{E^{1}} & \leq C_{1} \sup _{0<s \leq t}\left\{s^{\epsilon}\left\|u_{n}(s)-u_{m}(s)\right\|_{E^{1+\epsilon}}\right\} \\
& +C_{2}\left\|u_{n}-u_{m}\right\|_{E^{1}} \leq C\left\|u_{n}-u_{m}\right\|_{E^{1}} .
\end{aligned}
$$

Isto nos permite fazer $n \rightarrow \infty$ e obter soluções em $C\left(\left[0, \tau_{0}\right], E^{1}\right) \cap C\left(\left(0, \tau_{0}\right], E^{1+\epsilon}\right)$ com condições iniciais em $E^{1}$.

Da discussão acima, parece razoável dar as seguintes definições:

Definição 1 Dizemos que $e:\left[t_{0}, \tau\right] \rightarrow E^{1}$ é uma solução fraca e-regular para (1) se e $\in$ $C\left(\left[t_{0}, \tau\right], E^{1}\right) \cap C\left(\left(t_{0}, \tau\right], E^{1+\epsilon}\right)$, e e $(t)$ satisfaz

$$
e(t)=\mathbf{e}^{A\left(t-t_{0}\right)} e_{0}+\int_{t_{0}}^{t} \mathbf{e}^{A(t-s)} f(s, e(s)) d s .
$$

Definição 2 Para $\epsilon \geq 0$, diremos que a transformação $g$, é uma transformação $\epsilon$-regular relativamente ao par $\left(E^{1}, E\right)$, se existe $\rho>1, \gamma(\epsilon)$ com $\rho \epsilon \leq \gamma(\epsilon)<1$ e uma constante $c$, tal que $g: E^{1+\epsilon} \rightarrow E^{\gamma(\epsilon)} e$

$$
\|g(u)-g(v)\|_{E^{\gamma(\epsilon)}} \leq c\|u-v\|_{E^{1+\epsilon}}\left(\|u\|_{E^{1+\epsilon}}^{\rho-1}+\|v\|_{E^{1+\epsilon}}^{\rho-1}+1\right),
$$

$u, v \in E^{1+\epsilon}$.

Em [5], é provado que se $f(\cdot)$ é uma transformação $\epsilon$-regular, para algum $\epsilon>0$, então existe uma única solução $\epsilon$-regular para (1). Isto significa que quando lidamos com existência e unicidade para uma equação particular no caso de não linearidades críticas, devemos nos concentrar nos dois pontos seguintes:

(i) Entender a escala de espaços de potência fracionária associada a parte linear $A$, especialmente as suas imersões em espaços conhecidos como os espaços $L^{p}$ (que no exemplo é dada por $(5))$.

(ii) Estudar as propriedades de $\epsilon$-regularidade da não-linearidade $f$ nesta escala de espaços. Isto é conseguido, em geral, aplicando-se as imersões de Sobolev e as desigualdades de Hölder.

Uma vez que (i) e (ii) foram estudados, podemos aplicar os resultados de [5] para obter a existência local. 
Como vimos na ilustração acima, é de fundamental importância conhecer como os espaços de potências fracionárias associados ao operador setorial envolvido se relacionam com espaços conhecidos (parte principal da equação) (esta relação é dada por (5)) no exemplo). O conhecimento desses espaços; isto é, o conhecimento de suas relações com espaços conhecidos, determinam a classe de não linearidade para o qual o problema parabólico em questão é localmente bem posto. O objetivo deste trabalho é estudar resultados de T. Kato e H. Triebel com a finalidade de conhecer relações entre os espaços de potências fracionárias e espaços de interpolação (este último grupo de espaços tem relações bastante razoáveis com espaços conhecidos).

Com este objetivo, organizamos este trabalho da seguinte forma: No Capítulo 1, apresentamos várias definições e um bom número de resultados que são importantes para o desenvolvimento deste trabalho, deixamos de demonstrar vários dos resultados apresentados a fim de tornar a leitura menos cansativa. Os Capítulos 2 e 3, contêm um estudo detalhado da teoria de potências fracionárias, onde são apresentados alguns resultados importantes devido a T. Kato [15, 16, 17], bem como outros resultados de grande relevância. No Capítulo 4, estudamos os espaços de interpolação complexa e apresentamos um resultado devido a H. Triebel [21]. Finalmente, no Capítulo 5, com as ferramentas adquiridas nos Capítulos 2, 3 e 4, finalizamos este trabalho, relacionando os espaços de potências fracionárias com os espaços de interpolação complexa. 


\section{Capítulo 1}

\section{Preliminares}

Neste primeiro capítulo iremos apresentar algumas definições e resultados que serão de grande importância para o desenvolvimento deste trabalho. Por comodidade, omitiremos algumas das demostrações que podem ser encontradas em [8, 13, 19].

\subsection{Semigrupos e Seus Geradores}

Recorde que se $E$ e $F$ são espaços de Banach sobre um corpo $\mathbb{K}(\mathbb{K}=\mathbb{R}$ ou $\mathbb{K}=\mathbb{C})$ denotamos por $L(E, F)$ o espaço dos operadores lineares e contínuos de $E$ em $F$ com a norma usual; isto é, para $T \in L(E, F)$,

$$
\|T\|_{L(E, F)}=\sup _{\substack{e \in E \\ e \neq 0}} \frac{\|T e\|_{F}}{\|e\|_{E}} .
$$

No caso particular, em que $E=F$ escrevemos $L(E)$ para denotar $L(E, E)$. Seja $E^{*}$ o dual topológico de $E$; isto é, $E^{*}=L(E, \mathbb{K})$ com a topologia dada pela norma acima. Denotamos o valor de $e^{*} \in E^{*}$ em $e \in E$ por $\left\langle e^{*}, e\right\rangle$ ou $\left\langle e, e^{*}\right\rangle$.

Definição 1.1.1 Um semigrupo de operadores lineares em $E$ é uma família $\{T(t): t \geq 0\} \subset$ $L(E)$ tal que

(i) $T(0)=I_{E}$,

(ii) $T(t+s)=T(t) T(s)$, para todo $t, s \geq 0$.

Se adicionalmente

(iii) $\left\|T(t)-I_{E}\right\|_{L(E)} \rightarrow 0$ quando $t \rightarrow 0^{+}$, dizemos que o semigrupo é uniformemente contínuo

(iv) $\|T(t) e-e\|_{E} \rightarrow 0$ quando $t \rightarrow 0^{+}, \forall e \in E$, dizemos que o semigrupo é fortemente contínuo. 
Todo semigrupo fortemente contínuo possui uma dominação exponencial que é dada no teorema a seguir.

Teorema 1.1.1 Suponha que $\{T(t): t \geq 0\} \subset L(E)$ é um semigrupo fortemente contínuo. Então, existe $M \geq 1$ e $\beta \in \mathbb{R}$ tais que

$$
\|T(t)\|_{L(E)} \leq M \mathbf{e}^{\beta t}, \quad \forall t \geq 0 .
$$

Para qualquer $\ell>0$ podemos escolher $\beta \geq \frac{1}{\ell} \log \|T(\ell)\|_{L(E)}$ e então escolher $M$.

Definição 1.1.2 Seja $A: D(A) \subset E \longrightarrow E$ um operador linear, o conjunto

$$
\left\{\lambda \in \mathbb{C}: \overline{R(\lambda I-A)}=E,(\lambda I-A)^{-1} \text { existe e é limitado sobre } R(\lambda I-A)\right\}
$$

é chamado conjunto resolvente do operador $A$ e é denotado por $\rho(A)$. O conjunto $\sigma(A)=$ $\mathbb{C} / \rho(A)$ é chamado espectro do operador $A$.

Definição 1.1.3 Se $\{T(t): t \geq 0\} \subset L(E)$ é um semigrupo fortemente contínuo de operadores lineares, seu gerador infinitesimal é o operador definido por $A: D(A) \subset E \rightarrow E$, onde

$$
D(A)=\left\{e \in E: \lim _{t \rightarrow 0^{+}} \frac{T(t) e-e}{t} \text { existe }\right\}, \quad A e=\lim _{t \rightarrow 0^{+}} \frac{T(t) e-e}{t} .
$$

O resultado a seguir coleta alguns fatos importantes sobre semigrupos fortemente contínuos.

Teorema 1.1.2 Suponha que $\{T(t): t \geq 0\} \subset L(E)$ é um semigrupo fortemente contínuo.

1. Para qualquer $e \in E, t \mapsto T(t) e$ é contínuo para $t \geq 0$.

2. $t \mapsto\|T(t)\|_{L(E)}$ é semicontínua inferiormente e portanto mensurável.

3. Seja A o gerador infinitesimal de $T(t)$; então, A é densamente definido e fechado. Para $e \in D(A), t \mapsto T(t) e$ é continuamente diferenciável $e$

$$
\frac{d}{d t} T(t) e=A T(t) e=T(t) A e, \quad t>0 .
$$

4. $\cap_{m \geq 1} D\left(A^{m}\right)$ é denso em $E$.

5. Para $\operatorname{Re} \lambda>\beta$ e $\beta$ dado no Teorema 1.1.1, $\lambda$ está no resolvente $\rho(A)$ de $A$ e

$$
(\lambda-A)^{-1} e=\int_{0}^{\infty} \mathbf{e}^{-\lambda t} T(t) e d t, \quad \forall e \in E .
$$

Teorema 1.1.3 (O Segundo Limite Fundamental) Seja $\{T(t): t \geq 0\}$ um semigrupo fortemente contínuo em E. Se A é o seu gerador infinitesimal, então

$$
T(t) e=\lim _{n \rightarrow \infty}\left(I-\frac{t}{n} A\right)^{-n} e=\lim _{n \rightarrow \infty}\left[\frac{n}{t}\left(\frac{n}{t}-A\right)^{-1}\right]^{n} e, \quad \forall e \in E
$$

e os limites são uniformes para $t$ em intervalos limitados de $\mathbb{R}$. 


\subsection{O Teorema de Hille-Yosida}

Teorema 1.2.1 (Hille-Yosida) Suponha que $A: D(A) \subset E \rightarrow E$ é um operador linear. Então os fatos seguintes são equivalentes

(i) A é o gerador infinitesimal de um semigrupo fortemente contínuo $\{T(t): t \geq 0\} \subset L(E)$ tal que

$$
\|T(t)\|_{L(E)} \leq \mathbf{e}^{\omega t}, \quad \forall t \geq 0
$$

(ii) A é um operador linear fechado, densamente definido cujo conjunto resolvente contém $(\omega, \infty) e$

$$
\left\|(\lambda-A)^{-1}\right\|_{L(E)} \leq \frac{1}{\lambda-\omega}, \quad \forall \lambda>\omega
$$

Ambas as condições (i) e (ii) dependem da escolha da norma em E. Daremos uma formulação independente da norma, mas na prática devemos usualmente procurar normas especiais para a qual este teorema se aplique.

Lema 1.2.1 Suponha que A é um operador linear cujo conjunto resolvente contém $(0, \infty)$ e que satisfaz

$$
\left\|(\lambda-A)^{-n}\right\|_{L(E)} \leq M \lambda^{-n}, \quad n \geq 1, \lambda>0
$$

Então existe uma norma $|\cdot|_{E}$ em E tal que

$$
\|e\|_{E} \leq|e|_{E} \leq M\|e\|_{E}, \quad \forall e \in E
$$

e

$$
\left|(\lambda-A)^{-1} e\right|_{E} \leq \lambda^{-1}|e|_{E}, \quad \forall e \in E, \lambda>0
$$

Teorema 1.2.2 (Forma Geral do Teorema de Hille-Yosida) Seja $A: D(A) \subset E \rightarrow E$ um operador linear. As seguintes afirmativas são equivalente

(i) A é o gerador infinitesimal de um semigrupo fortemente contínuo $\{T(t): t \geq 0\} \subset L(E)$ tal que

$$
\|T(t)\|_{L(E)} \leq M \mathbf{e}^{\beta t}, \quad \forall t \geq 0
$$

(ii) A é fechado, densamente definido, o conjunto resolvente de $A$ contém $(\beta, \infty)$ e

$$
\left\|(\lambda-A)^{-n}\right\|_{L(E)} \leq M(\lambda-\beta)^{-n}, \quad \forall \lambda>\beta, n=1,2, \cdots
$$




\subsection{O Teorema de Lumer-Phillips}

Definição 1.3.1 Seja E um espaço de Banach sobre $\mathbb{K}$ com norma $\|\cdot\|_{E}$ e seja $E^{*}=L(E, \mathbb{K})$ o seu dual topológico com a norma usual $\|\cdot\|_{E^{*}}\left(\left\|e^{*}\right\|_{E^{*}}=\sup \left\{\operatorname{Re}\left\langle e^{*}, e\right\rangle:\|e\|_{E} \leq 1\right\}\right)$. A aplicação dualidade $J: E \rightarrow 2^{E^{*}}$ é uma função multivoca definida por

$$
J(e)=\left\{e^{*} \in E^{*}: \operatorname{Re}\left\langle e^{*}, e\right\rangle=\|e\|_{E}^{2},\left\|e^{*}\right\|_{E^{*}}=\|e\|_{E}\right\}
$$

$J(e) \neq \varnothing$, pelo Teorema de Hahn-Banach.

Definição 1.3.2 Um operador linear $A: D(A) \subset E \rightarrow E$ é dissipativo se para cada e $\in D(A)$ existe $e^{*} \in J(e)$ tal que $\operatorname{Re}\left\langle e^{*}, A e\right\rangle \leq 0$.

Observação 1.3.1 Se $\{T(t): t \geq 0\} \subset L(E)$ é um semigrupo fortemente contínuo tal que

$$
\|T(t)\| \leq 1, \forall t \leq 0
$$

então, dizemos que $T(t)$ é um semigrupo fortemente contínuo de contrações.

Lema 1.3.1 O operador linear A é dissipativo se e somente se

$$
\|(\lambda-A) e\|_{E} \geq \lambda\|e\|_{E}
$$

para todo $e \in D(A)$ e $\lambda>0$.

Teorema 1.3.1 (Lumer-Phillips) Suponha que A é um operador linear densamente definido em um espaço de Banach E.

(i) Se A é o gerador infinitesimal de um semigrupo fortemente contínuo de contrações, então $A$ é dissipativo (de fato, $\operatorname{Re}\left\langle e^{*}, A e\right\rangle \leq 0$ para todo $\left.e^{*} \in J(e)\right)$ e $R(\lambda-A)=E$ para todo $\lambda>0$,

(ii) Se $A$ é dissipativo e $R\left(\lambda_{0}-A\right)=E$ para algum $\lambda_{0}>0$, então $A$ é o gerador de um semigrupo fortemente contínuo de contrações.

Prova. (i) Se $A$ gera $\{T(t): t \geq 0\},\|T(t)\|_{L(E)} \leq 1$ então $R(\lambda-A)=E$ para todo $\lambda>0$ pelo Teorema de Hille-Yosida e para qualquer $e \in E, e^{*} \in J(e), t>0$,

$$
\left|\left\langle e^{*}, T(t) e\right\rangle\right| \leq\left\|e^{*}\right\|_{E^{*}}\|T(t) e\|_{E} \leq\|e\|_{E}^{2}
$$


então

$$
\operatorname{Re}\left\langle e^{*}, \frac{T(t) e-e}{t}\right\rangle=\frac{1}{t}\left\{\operatorname{Re}\left\langle e^{*}, T(t) e\right\rangle-\|e\|_{E}^{2}\right\} \leq 0 .
$$

Portanto se $e \in D(A), \operatorname{Re}\left\langle e^{*}, A e\right\rangle \leq 0$.

(ii) Se $\lambda>0$ e $e \in D(A)$, do Lema 1.3.1 temos que

$$
\|(\lambda-A) e\|_{E} \geq \lambda\|e\|_{E}
$$

Agora $R\left(\lambda_{0}-A\right)=E,\left\|\left(\lambda_{0}-A\right) e\right\|_{E} \geq \lambda_{0}\|e\|_{E}$ para $e \in D(A)$, logo $\lambda_{0}$ está no conjunto resolvente de $A$ e $A$ é fechado. Seja $\Lambda=\{\lambda \in \rho(A) \cap \mathbb{R}: \lambda>0\}$. $\Lambda$ é um conjunto aberto em $(0, \infty)$ já que $\rho(A)$ é aberto, provaremos que $\Lambda$ é também fechado em $(0, \infty)$ para concluir que $\Lambda=(0, \infty)$. Suponha que $\left\{\lambda_{n}\right\}_{n=1}^{\infty} \subset \Lambda, \lambda_{n} \rightarrow \lambda>0$, se $n$ é suficientemente grande temos que $\left|\lambda_{n}-\lambda\right| \leq \lambda / 4$ então, $\left\|\left(\lambda-\lambda_{n}\right)\left(\lambda_{n}-A\right)^{-1}\right\|_{L(E)} \leq\left|\lambda_{n}-\lambda\right| \lambda_{n}^{-1} \leq 1 / 3$ e $I+\left(\lambda-\lambda_{n}\right)\left(\lambda_{n}-A\right)^{-1}$ é um isomorfismo de $E$. Logo

$$
\lambda-A=\left\{I+\left(\lambda-\lambda_{n}\right)\left(\lambda_{n}-A\right)^{-1}\right\}\left(\lambda_{n}-A\right)
$$

leva $D(A)$ sobre $E$ e $\lambda \in \rho(A)$, como queríamos.

Agora todas as hipóteses do Teorema de Hille-Yosida (ii) estão verificadas e a prova está completa.

A seguir recordamos a definição de operadores adjuntos. Seja $E$ um espaço de Banach com dual $E^{*}$. Seja $S: D(S) \subset E \rightarrow E$ um operador linear com domínio denso. O adjunto $S^{*}: D\left(S^{*}\right) \subset E^{*} \rightarrow E^{*}$ de $S$ é o operador linear definido por: $D\left(S^{*}\right)$ é o conjunto dos $e^{*} \in E^{*}$ para os quais existe $f^{*} \in E^{*}$ com

$$
\left\langle e^{*}, S e\right\rangle=\left\langle f^{*}, e\right\rangle \quad \forall e \in D(S)
$$

e se $e^{*} \in D\left(S^{*}\right)$ então $f^{*}=S^{*} e^{*}$ onde $f^{*}$ é o elemento de $E^{*}$ satisfazendo (1.3). Note que como $D(S)$ é denso em $E$ existe no máximo um $f^{*} \in E^{*}$ para o qual (1.3) vale.

Seja $H$ um espaço de Hilbert com produto escalar $\langle\cdot, \cdot\rangle$. Identificamos $H$ e $H^{*}$ e denotamos ambos por $H$.

Definição 1.3.3 Seja $H$ um espaço de Hilbert com produto interno $\langle\cdot, \cdot\rangle$. Um operador $A$ : $D(A) \subset H \rightarrow H$ é simétrico se $\overline{D(A)}=H$ e $A \subset A^{*}$; isto é, $\langle A e, f\rangle=\langle e, A f\rangle$ para todo $e, f \in D(A)$. A é auto-adjunto se $A=A^{*}$.

Corolário 1.3.1 Seja A um operador linear fechado e densamente definido. Se ambos $A$ e $A^{*}$ são dissipativos, então $A$ é o gerador infinitesimal de um semigrupo fortemente contínuo de contrações sobre E. 
Em muitos exemplos a técnica utilizada para verificar as estimativas espectrais necessárias para se garantir que um operador $A$ é o gerador de um semigrupo fortemente contínuo, são obtidas através do conhecimento da chamada imagem numérica que é definida a seguir.

Se $A$ é um operador linear em um espaço de Banach complexo $E$, a sua imagem numérica $W(A)$ é o conjunto

$$
W(A):=\left\{\left\langle e^{*}, A e\right\rangle: e \in D(A),\|e\|_{E}=1, e^{*} \in E^{*},\left\|e^{*}\right\|_{E^{*}}=1,\left\langle e^{*}, e\right\rangle=1\right\} .
$$

No caso em que $E$ é um espaço de Hilbert $W(A)=\left\{\langle A e, e\rangle: e \in D(A),\|e\|_{E}=1\right\}$.

Teorema 1.3.2 Seja $A: D(A) \subset E \rightarrow E$ um operador fechado densamente definido. Seja $W(A)$ a imagem numérica de $A$ e $\Sigma$ um subconjunto aberto e conexo em $\mathbb{C} \backslash W(A)$. Se $\lambda \notin \overline{W(A)}$ então $\lambda-A$ é um-a-um e tem imagem fechada e satisfaz

$$
\|(\lambda-A) e\|_{L(E)} \geq d(\lambda, W(A))\|e\|_{E} .
$$

Adicionalmente, se $\rho(A) \cap \Sigma \neq \varnothing$ então $\rho(A) \supset \Sigma e$

$$
\left\|(\lambda-A)^{-1}\right\|_{L(E)} \leq \frac{1}{d(\lambda, W(A))}, \quad \forall \lambda \in \Sigma
$$

onde $d(\lambda, W(A))$ é a distância de $\lambda$ a $W(A)$.

Definição 1.3.4 Dizemos que um operador $A: D(A) \subset E \longrightarrow E$ é fechável se $\overline{G r a f(A)}$ é gráfico de um operador $\bar{A} . \bar{A}$ é o menor operador fechado que estende $A$.

Teorema 1.3.3 Seja A um operador dissipativo em $E$

(a) Se para algum $\lambda_{0}>0, R\left(\lambda_{0}-A\right)=E$ então $R(\lambda-A)=E$ para todo $\lambda>0$.

(b) Se A é fechável então o seu fecho $\bar{A}$ é também dissipativo.

(c) Se $\overline{D(A)}=E$ então A é fechável.

Prova. A afirmativa (a) foi provada no Teorema de Lumer-Phillips. Para provar (b) seja $e \in$ $D(\bar{A}), f=\bar{A} e$. Então existe uma seqüência $\left\{e_{n}\right\} \subset D(A)$ tal que $e_{n} \rightarrow e$ e $A e_{n} \rightarrow f=\bar{A} e$. Do Lema 1.3.1 segue que $\left\|\lambda e_{n}-A e_{n}\right\|_{E} \geq \lambda\left\|e_{n}\right\|_{E}$, para $\lambda>0$ e fazendo $n \rightarrow \infty$ temos

$$
\|\lambda e-\bar{A} e\|_{E} \geq \lambda\|e\|_{E}, \quad \lambda>0 .
$$

Como (1.7) vale para todo $e \in D(\bar{A}), \bar{A}$ é dissipativo pelo Lema 1.3.1. Para provar $(c)$ assuma que $A$ não é fechável. Então existe uma seqüência $\left\{e_{n}\right\} \subset D(A), e_{n} \rightarrow 0$ e $A e_{n} \rightarrow f$ com $\|f\|_{E}=1$. Do Lema 1.3.1 segue que para todo $t>0$ e $e \in D(A)$

$$
\left\|\left(e+t^{-1} e_{n}\right)-t A\left(e+t^{-1} e_{n}\right)\right\|_{E} \geq\left\|e+t^{-1} e_{n}\right\|_{E} .
$$


Fazendo $n \rightarrow \infty$ e então $t \rightarrow 0$ resulta $\|e-f\|_{E} \geq\|e\|_{E}$ para todo $e \in D(A)$. Mas isto é impossível se $D(A)$ é denso em $E$ e portanto $A$ é fechável.

Teorema 1.3.4 Seja A dissipativo com $R(I-A)=E$. Se E é reflexivo então $\overline{D(A)}=E$.

\subsection{Pseudo-Resolventes}

Seja $A$ um operador fechado e densamente definido em $E$. Se $\mu$ e $\lambda$ estão em $\rho(A)$, então temos

$$
(\lambda-A)^{-1}-(\mu-A)^{-1}=(\mu-\lambda)(\lambda-A)^{-1}(\mu-A)^{-1} .
$$

De fato,

$$
\begin{aligned}
(\mu-\lambda)(\lambda-A)^{-1}(\mu-A)^{-1} & =\mu(\lambda-A)^{-1}(\mu-A)^{-1}-\lambda(\lambda-A)^{-1}(\mu-A)^{-1} \\
& =\left[\mu(\lambda-A)^{-1}-\lambda(\lambda-A)^{-1}\right](\mu-A)^{-1} \\
& =\left[\mu(\lambda-A)^{-1}-\left[I+A(\lambda-A)^{-1}\right]\right](\mu-A)^{-1} \\
& =\left[-I+(\lambda-A)^{-1}(\mu-A)\right](\mu-A)^{-1} \\
& =(\lambda-A)^{-1}-(\mu-A)^{-1} .
\end{aligned}
$$

Motivado por isto definimos

Definição 1.4.1 Seja $\Delta$ um subconjunto do plano complexo. Uma família $\{J(\lambda), \lambda \in \Delta\}$, de operadores lineares limitados em E satisfazendo

$$
J(\lambda)-J(\mu)=(\mu-\lambda) J(\lambda) J(\mu), \quad \lambda, \mu \in \Delta
$$

é chamado um pseudo-resolvente em $\Delta$.

O objetivo final desta seção é determinar condições sob as quais existe um operador fechado e densamente definido $A$ tal que $J(\lambda)$ é o resolvente de $A$.

Lema 1.4.1 Seja $\Delta$ um subconjunto de $\mathbb{C}$. Se $J(\lambda)$ é pseudo-resolvente em $\Delta$, então $J(\lambda) J(\mu)=$ $J(\mu) J(\lambda)$. O núcleo $N(J(\lambda))$ e a imagem $R(J(\lambda))$ são independentes de $\lambda \in \Delta . N(J(\lambda))$ é um subespaço fechado de E.

Prova. Por (1.8) segue que $J(\lambda)$ e $J(\mu)$ comutam para $\lambda, \mu \in \Delta$. Vejamos, se $\lambda \neq \mu$,

$$
J(\lambda) J(\mu)=\frac{J(\lambda)-J(\mu)}{\lambda-\mu}=\frac{J(\mu) J(\lambda)}{\mu-\lambda}=J(\mu) J(\lambda)
$$


Também, reescrevendo (1.8) na forma

$$
J(\lambda)=J(\mu)[I+(\mu-\lambda) J(\lambda)]
$$

obtemos que $R(J(\mu)) \supset R(J(\lambda))$ e por simetria temos a igualdade. Semelhantemente $N(J(\lambda))=$ $N(J(\mu))$. Como $N(J(\lambda))$ é um operador linear limitado para cada $\lambda \in \Delta$, segue que $N(J(\lambda)) \subset E$ é um subespaço fechado.

Teorema 1.4.1 Seja $\Delta$ um subconjunto de $\mathbb{C}$ e seja $J(\lambda)$ pseudo-resolvente em $\Delta$. Então, $J(\lambda)$ é o resolvente de um operador linear fechado densamente definido se e somente se $N(J(\lambda))=\{0\}$ e $R(J(\lambda))$ é denso em $E$.

Prova. Se $J(\lambda)$ é o resolvente de um operador fechado e densamente definido $A$, então $J(\lambda)=$ $(\lambda I-A)^{-1}: R(\lambda I-A) \rightarrow D(A)$. Logo, $N(J(\lambda))=\{0\}$ e $R(J(\lambda))=D(A)$ é denso em $E$. Por outro lado, assuma que $N(J(\lambda))=\{0\}$ e $R(J(\lambda))=D(A)$ é denso em $E$. De $N(J(\lambda))=\{0\}$ segue que $J(\lambda)$ é um-a-um. Seja $\lambda_{0} \in \Delta$ e defina

$$
A=\lambda_{0} I-J\left(\lambda_{0}\right)^{-1}: R\left(J\left(\lambda_{0}\right)\right) \longrightarrow E .
$$

Note que $A$ está bem definido, pois para cada $e \in R(J(\lambda))$,

$$
J(\lambda) J(\mu)\left[J(\mu)^{-1} e-J(\lambda)^{-1} e\right]=J(\lambda) e-J(\mu) e=(\mu-\lambda) J(\lambda) J(\mu) e
$$

assim,

$$
J(\mu)^{-1} e-J(\lambda)^{-1} e=(\mu-\lambda) e
$$

e portanto,

$$
\lambda e-J(\lambda)^{-1} e=\mu e-J(\mu)^{-1} e
$$

ou seja, $\lambda_{0}-J\left(\lambda_{0}\right)^{-1}$ é independente de $\lambda_{0}$. Observe que $A$ assim definido é claramente linear, fechado, pois $J\left(\lambda_{0}\right)$ é linear limitado, ainda, $A$ é densamente definido, já que $D(A)=R\left(J\left(\lambda_{0}\right)\right)$. Da definição de $A$ é claro que

$$
\left(\lambda_{0} I-A\right) J\left(\lambda_{0}\right)=J\left(\lambda_{0}\right)\left(\lambda_{0} I-A\right)=I
$$

e portanto $J\left(\lambda_{0}\right)=\left(\lambda_{0} I-A\right)^{-1}$. Se $\lambda \in \Delta$ então

$$
\begin{aligned}
(\lambda I-A) J(\lambda) & =\left(\left(\lambda-\lambda_{0}\right) I+\left(\lambda_{0} I-A\right)\right) J(\lambda) \\
& =\left(\left(\lambda-\lambda_{0}\right) I+\left(\lambda_{0} I-A\right)\right) J\left(\lambda_{0}\right)\left[I-\left(\lambda-\lambda_{0}\right) J(\lambda)\right] \\
& =I+\left(\lambda-\lambda_{0}\right)\left[J\left(\lambda_{0}\right)-J(\lambda)-\left(\lambda-\lambda_{0}\right) J\left(\lambda_{0}\right) J(\lambda)\right] \\
& =I
\end{aligned}
$$


e semelhantemente $J(\lambda)(\lambda I-A)=I$. Portanto $J(\lambda)=(\lambda-A)^{-1}$ para todo $\lambda \in \Delta$. Em particular $A$ é unicamente determinado por $J(\lambda)$.

A seguir damos condições suficientes para que pseudo-resolventes sejam resolventes.

Teorema 1.4.2 Seja $\Delta \subset \mathbb{C}$ ilimitado e seja $J(\lambda)$ um pseudo-resolvente em $\Delta$. Se $R(J(\lambda))$ é denso em E e existe uma seqüência $\lambda_{n} \in \Delta$ com $\left|\lambda_{n}\right| \rightarrow \infty e$

$$
\left\|\lambda_{n} J\left(\lambda_{n}\right)\right\|_{L(E)} \leq M
$$

para alguma constante $M$, então $J(\lambda)$ é o resolvente de um único operador fechado e densamente definido.

Prova. De (1.9) segue que $\left\|J\left(\lambda_{n}\right)\right\|_{L(E)} \rightarrow 0$ quando $n \rightarrow \infty$. Seja $\mu \in \Delta$. De (1.8) deduzimos que

$$
\left\|\left(\lambda_{n} J\left(\lambda_{n}\right)-I\right) J(\mu)\right\|_{L(E)}=\left\|(\mu J(\mu)-I) J\left(\lambda_{n}\right)\right\|_{L(E)} \rightarrow 0, \quad n \rightarrow \infty .
$$

Portanto, se $e \in R(J(\mu))$ temos

$$
\lambda_{n} J\left(\lambda_{n}\right) e \rightarrow e, \quad n \rightarrow \infty
$$

Como $R(J(\mu))$ é denso em $E$ e $\lambda_{n} J\left(\lambda_{n}\right)$ é uniformemente limitada, temos que (1.10) vale para todo $e \in E$. Se $e \in N(J(\lambda))$ então $\lambda_{n} J\left(\lambda_{n}\right) e=0$ e de (1.10) deduzimos que $e=0$. Portanto $N(J(\lambda))=\{0\}$ e, do Teorema 1.4.1, $J(\lambda)$ é o resolvente de um operador fechado e densamente definido $A$.

Corolário 1.4.1 Seja $\Delta \subset \mathbb{C}$ ilimitado e $J(\lambda)$ um pseudo-resolvente em $\Delta$. Se existe uma seqüência $\lambda_{n} \in \Delta$ tal que $\left|\lambda_{n}\right| \rightarrow \infty$ quando $n \rightarrow \infty e$

$$
\lim _{n \rightarrow \infty} \lambda_{n} J\left(\lambda_{n}\right) e=e, \quad \forall e \in E
$$

então $J(\lambda)$ é o resolvente de um operador (unicamente definido) fechado e densamente definido A.

Prova. Do Princípio da Limitação Uniforme e de (1.11) seque que (1.9) vale. Do Lema 1.4.1 sabemos que $R(J(\lambda))$ é independente de $\lambda \in \Delta$ e portanto (1.11) implica que $R(J(\lambda))$ é denso em E. Portanto, as condições do Teorema 1.4.2 estão satisfeitas e o resultado segue. 


\subsection{O Semigrupo Dual e o Teorema de Stone}

Começamos com alguns resultados básicos sobre operadores duais.

Lema 1.5.1 Seja $S \in L(E)$; então, $S^{*} \in L\left(E^{*}\right)$ e $\|S\|_{L(E)}=\left\|S^{*}\right\|_{L(E)}$.

Lema 1.5.2 Seja A um operador linear densamente definido em $E$. Se $\lambda \in \rho(A)$ então $\lambda \in \rho\left(A^{*}\right)$ $e$

$$
\left(\lambda-A^{*}\right)^{-1}=\left((\lambda-A)^{-1}\right)^{*}
$$

Seja $\{T(t): t \geq 0\}$ um semigrupo fortemente contínuo em $E$. Para $t>0$ seja $\left\{T(t)^{*}: t \geq 0\right\}$ o semigrupo dual. O semigrupo dual não precisa ser fortemente contínuo em $E^{*}$.

Definição 1.5.1 Seja $S$ um operador linear em E e seja $F$ um subespaço de E. O operador $\tilde{S}$ definido por $D(\tilde{S})=\{e \in D(S) \cap F: S e \in F\}$ e $\tilde{S} e=$ Se para e $\in D(\tilde{S})$ é chamado parte de $S$ em $F$.

Teorema 1.5.1 Seja $\{T(t): t \geq 0\}$ um semigrupo fortemente contínuo em E com gerador infinitesimal $A$ e $\left\{T(t)^{*}: t \geq 0\right\}$ o semigrupo dual. Se $A^{*}$ é o adjunto de $A$ e $E^{\odot}$ é o fecho de $D\left(A^{*}\right)$ em $E^{*}$, então a restrição $\left\{T(t)^{\odot}: t \geq 0\right\}$ de $\left\{T(t)^{*}: t \geq 0\right\}$ a $E^{\odot}$ é um semigrupo fortemente contínuo em $E^{\odot}$. O gerador infinitesimal $A^{\odot}$ de $\left\{T(t)^{\odot}: t \geq 0\right\}$ é a parte de $A^{*}$ em $E^{\odot}$.

Prova. Como $A$ é o gerador infinitesimal de $\{T(t): t \geq 0\}$, existem constantes $\beta$ e $M$ tais que para todo $\lambda>\omega, \lambda \in \rho(A) \mathrm{e}$

$$
\left\|(\lambda-A)^{-n}\right\|_{L(E)} \leq \frac{M}{(\lambda-\omega)^{n}}, \quad n=1,2, \cdots
$$

Segue que $\lambda \in \rho\left(A^{*}\right)$ e

$$
\left\|\left(\lambda I^{*}-A^{*}\right)^{-n}\right\|_{L\left(E^{*}\right)} \leq \frac{M}{(\lambda-\omega)^{n}}, \quad n=1,2, \cdots
$$

Seja $J(\lambda)$ a restrição de $\left(\lambda I^{*}-A^{*}\right)^{-1}$ a $E^{\odot}$. Segue que

$$
\left\|J(\lambda)^{n}\right\|_{L(E \odot)} \leq \frac{M}{(\lambda-\omega)^{n}} .
$$

Observe que $A^{*}$ é fechado e $E^{\odot}=\overline{D\left(A^{*}\right)}$ em $E^{*}$, e mais,

$$
J(\lambda)-J(\mu)=(\mu-\lambda) J(\lambda) J(\mu), \quad \lambda, \mu>\omega
$$


Exatamente como na prova do Teorema de Hille-Yosida, temos que

$$
\lambda J(\lambda)=\left(I^{*}-\lambda^{-1} A^{*}\right)^{-1}=I^{*}+A^{*} J(\lambda)
$$

Para $e \in D\left(A^{*}\right)$, segue

$$
\begin{aligned}
\left\|\lambda J(\lambda) e^{*}-e^{*}\right\|_{E} & =\left\|A^{*} J(\lambda) e^{*}\right\|_{E}=\|J(\lambda)\|_{L(E)}\left\|A^{*} e^{*}\right\|_{E} \\
& \leq \frac{M}{(\lambda-\omega)}\left\|A^{*} e^{*}\right\|_{E} \stackrel{\lambda \rightarrow \infty}{\longrightarrow} 0
\end{aligned}
$$

Como $\overline{D\left(A^{*}\right)}=E^{\odot}$, segue que

$$
\lim _{\lambda \rightarrow \infty} \lambda J(\lambda) e^{*} \rightarrow e^{*}, \quad \forall e^{*} \in E^{\odot} .
$$

Segue do Corolário 1.4.1 que $J(\lambda)$ é o resolvente de um operador fechado e densamente definido $A^{\odot}$ em $E^{\odot}$. Ainda, $A^{\odot}$ é o gerador infinitesimal de um semigrupo fortemente contínuo $\left\{T(t)^{\odot}\right.$ : $t \geq 0\}$ em $E^{\odot}$. Para $e \in E$ e $e^{\odot} \in E^{\odot}$ temos

$$
\left\langle e^{\odot},\left(I-\frac{t}{n} A\right)^{-n} e\right\rangle=\left\langle\left(I^{\odot}-\frac{t}{n} A^{\odot}\right)^{-n} e^{\odot}, e\right\rangle, \quad n=1,2,3 \cdots
$$

Fazendo $n \rightarrow \infty$ e usando o Teorema 1.1.3, obtemos

$$
\left\langle e^{\odot}, T(t) e\right\rangle=\left\langle T(t)^{\odot} e^{\odot}, e\right\rangle
$$

Segue que para $e^{\odot} \in E^{\odot}, T(t)^{*} e^{\odot}=T(t)^{\odot} e^{\odot}$ e $T(t)^{\odot}$ é a restrição de $T(t)^{*}$ a $E^{\odot}$.

Para concluir a prova temos que mostrar que $A^{\odot}$ é a parte de $A^{*}$ em $E^{\odot}$. Seja $e^{*} \in D\left(A^{*}\right)$ tal que $e^{*} \in E^{\odot}$ e $A^{*} e^{*} \in E^{\odot}$. Então $\left(\lambda I^{*}-A^{*}\right) e^{*} \in E^{\odot}$ e

$$
\left(\lambda I^{\odot}-A^{\odot}\right)^{-1}\left(\lambda I^{*}-A^{*}\right) e^{*}=e^{*}
$$

Portanto $e^{*} \in D\left(A^{\odot}\right)$ e aplicando $\lambda I^{\odot}-A^{\odot}$ em ambos os lados da igualdade acima temos $\left(\lambda I^{*}-A^{*}\right) e^{*}=\left(\lambda I^{\odot}-A^{\odot}\right) e^{*}$ e portanto $A^{\odot} e^{*}=A^{*} e^{*}$. Isto mostra que $A^{\odot}$ é a parte de $A^{*}$ em $E^{*}$.

O seguinte resultado identifica alguns casos em que o semigrupo dual é fortemente contínuo.

Lema 1.5.3 Seja $E$ um espaço de Banach reflexivo. Se $S: D(S) \subset E \rightarrow E$ é fechado e densamente definido então $D\left(S^{*}\right)$ é denso em $E^{*}$.

Corolário 1.5.1 Seja E um espaço de Banach reflexivo e $\{T(t): t \geq 0\}$ um semigrupo fortemente contínuo em $E$ com gerador infinitesimal $A$. O semigrupo dual $\left\{T(t)^{*}: t \geq 0\right\}$ de $\{T(t): t \geq 0\}$ é um semigrupo fortemente contínuo em $E^{*}$ cujo gerador infinitesimal é $A^{*}$. 
Uma vez que a restrição de $T(t)^{*}$ ao subespaço $X^{\odot}$ é um semigrupo fortemente contínuo, estamos exatamente na mesma posição que começamos. Em um espaço de Banach $X^{\odot}$ e com um semigrupo fortemente contínuo $\left\{T(t)^{\odot}: t \geq 0\right\}$ gerado pela parte $A^{\odot}$ de $A^{*}$ em $X^{\odot}$.

Podemos introduzir o espaço $X^{\odot *}$ e o semigrupo dual $T(t)^{\odot *}$ que é fortemente contínuo em $X^{\odot \odot}:=\overline{D\left(A^{\odot *}\right)}$.

A dualidade entre os elementos de $X$ e $X^{\odot}$ pode ser usada para definir uma imersão $j$ (note que $X^{\odot}$ é fraco-* denso em $\left.X^{*}\right)$ de $X$ em $X^{\odot *}$ com

$$
\left\langle j x, x^{\odot}\right\rangle_{X \odot *, X \odot}=\left\langle x^{\odot}, x\right\rangle_{X \odot, X}
$$

É claro que

$$
T(t)^{\odot *} j x=j(T(t) x)
$$

e portanto $j(X) \subset X^{\odot \odot}$. Sempre que $j(X)=X^{\odot \odot}$ chamaremos $X$ de $\odot$-reflexivo com respeito ao semigrupo $\{T(t): t \geq 0\}$.

Seja $H$ um espaço de Hilbert. Um operador limitado $U$ é unitário se $U^{*}=U^{-1}$. Recorde que $U$ é unitário se e somente se $R(U)=H$ e $U$ é uma isometria. De fato, se $U^{*} U=I$, então $x_{1}, x_{2} \in H$,

$$
\left\langle U x_{1}, U x_{2}\right\rangle=\left\langle x_{1}, U^{*} U x_{2}\right\rangle=\left\langle x_{1}, x_{2}\right\rangle
$$

tomando $x_{1}=x_{2}$ segue que $U$ é uma isometria e $R(U)=H$. Por outro lado, se $R(U)=H$ e $U$ é uma isometria, então $U$ é inversível e

$$
\left\langle U^{*} U x, x\right\rangle=\langle U x, U x\rangle=\|U x\|^{2}=\left\|x^{2}\right\|=\langle x, x\rangle, \quad \forall x \in H .
$$

Portanto, $U^{*} U=\mathrm{I}$.

Teorema 1.5.2 (Stone) Um operador A é o gerador infinitesimal de um grupo fortemente contínuo de operadores unitários em um espaço de Hilbert $H$ se e somente se $i A$ é auto-adjunto.

Prova. Se $A$ é o gerador de um grupo fortemente contínuo de operadores unitários $\{U(t): t \in \mathbb{R}\}$, então $A$ é densamente definido e utilizando o Corolário 1.5.1 obtemos, para $x \in D(A)$,

$$
-A x=\lim _{t \rightarrow 0^{+}} \frac{U(-t) x-x}{t}=\lim _{t \rightarrow 0^{+}} \frac{U^{*}(t) x-x}{t}=A^{*} x
$$

o que implica $A=-A^{*}$ e portanto $(i A)^{*}=i A$ e $i A$ é auto-adjunto.

Se por outro lado $i A$ é auto adjunto então $A$ é densamente definido e $A=-A^{*}$. Portanto, para todo $x \in D(A)$ temos

$$
\langle A x, x\rangle=\left\langle x, A^{*} x\right\rangle=-\overline{\langle A x, x\rangle}
$$


e portanto $\operatorname{Re}\langle A x, x\rangle=0$ para todo $x \in D(A)$, isto é, $A$ é dissipativo. Como $A=-A^{*}$, $\operatorname{Re}\left\langle A^{*} x, x\right\rangle=0$ para todo $x \in D(A)=D\left(A^{*}\right)$ e também $A^{*}$ é dissipativo. Logo $A$ e $A^{*}$ são densamente definidos e fechados e como $A^{* *}=A$, do Corolário 1.3.1, ambos $A$ e $A^{*}=-A$ são geradores infinitesimais de semigrupos fortemente contínuos de contrações em $H$. Se $\left\{U_{+}(t)\right.$ : $t \geq 0\}$ e $\left\{U_{-}(t): t \geq 0\right\}$ são os semigrupos gerados por $A$ e $A^{*}$ respectivamente, definimos

$$
U(t)= \begin{cases}U(t), & t \geq 0 \\ U_{-}(-t), & t \leq 0\end{cases}
$$

Então $U(t)$ é um grupo. De fato: Como $A$ e $-A$ são geradores de semigrupos fortemente contínuos $U_{+}(t)$ e $U_{-}(-t)$ que comutam. Se $W(t)=U_{+}(t) U_{-}(-t)$ então para $x \in D(A)=D(-A)$,

$$
\frac{W(t) x-x}{t}=U_{-}(t) \frac{U_{+}(t) x-x}{t}+\frac{U_{-}(t) x-x}{t} \rightarrow A x-A x=0, \quad \text { quando } t \rightarrow 0^{+} .
$$

Portanto, para $x \in D(A)$ temos que $W(t) x=x, t \geq 0$. Como $D(A)$ é denso em $H$ e $W(t)$ é limitado temos que $W(t)=I$, ou seja, $U_{-}(-t)=\left(U_{+}(t)\right)^{-1}$. Com isto, obtemos

i) $\mathrm{U}(0)=\mathrm{I}$.

ii) $U(t+s)=U(t) U(s),-\infty<t, s<\infty$. Se $t, s>0$ ou $t, s<0$ ok. Seja $t<0<s$. Se $t+s \leq 0$, então

$$
U(s) U(t+s)=U_{+}(-s) U_{+}(t+s)=U_{+}(t)
$$

assim

$$
U(t+s)=U_{+}(-s)^{-1} U_{+}(t)=U_{-}(-s) U_{+}(t)=U(t) U(s)
$$

Por outro lado, se $t+s \leq 0$, então

$$
U(-s) U(t+s)=U_{-}(s) U_{-}(-t-s)=U_{-}(-t)
$$

assim

$$
U(t+s)=\left(U_{-}(s)\right)^{-1} U_{-}(-t)=U_{+}(s) U_{-t}=U(t) U(s)
$$

Como $U(t)^{-1}=U(-t),\|U(t)\| \leq 1,\|U(-t)\| \leq 1$, segue que $R(U(t))=H$ e $U(t)$ é uma isometria para todo $t$ e portanto $U(t)$ é um grupo unitário de operadores sobre $H$, como queríamos.

\subsection{Transformada Inversa de Laplace}

Vimos no Teorema 1.1.2, (5) que

$$
(\lambda-A)^{-1}=\int_{0}^{\infty} \mathbf{e}^{-\lambda t} T(t) d t
$$


se Re $\lambda$ é grande. Isto sugere que usando a transformada inversa de Laplace poderemos encontrar $T(t)$, conhecido $A$. No que se segue perseguiremos este objetivo.

Lema 1.6.1

(a) $\int_{-\infty}^{\infty} \frac{\operatorname{sen} t}{t} d t=\pi$

(b) Se $f: \mathbb{R} \rightarrow \mathbb{C}$ é tal que $f(t) /(1+|t|)$ é integrável em $\mathbb{R} e \int_{-1}^{1}\left|\frac{f(t)-f(0)}{t}\right| d t<\infty$, então

$$
\int_{-\infty}^{\infty} f(t) \frac{\operatorname{sen} N t}{\pi t} d t \rightarrow f(0) \quad \text { quando } \quad N \rightarrow+\infty
$$

Prova. (a) Note que se $\gamma$ é a curva da figura abaixo no plano complexo, temos que

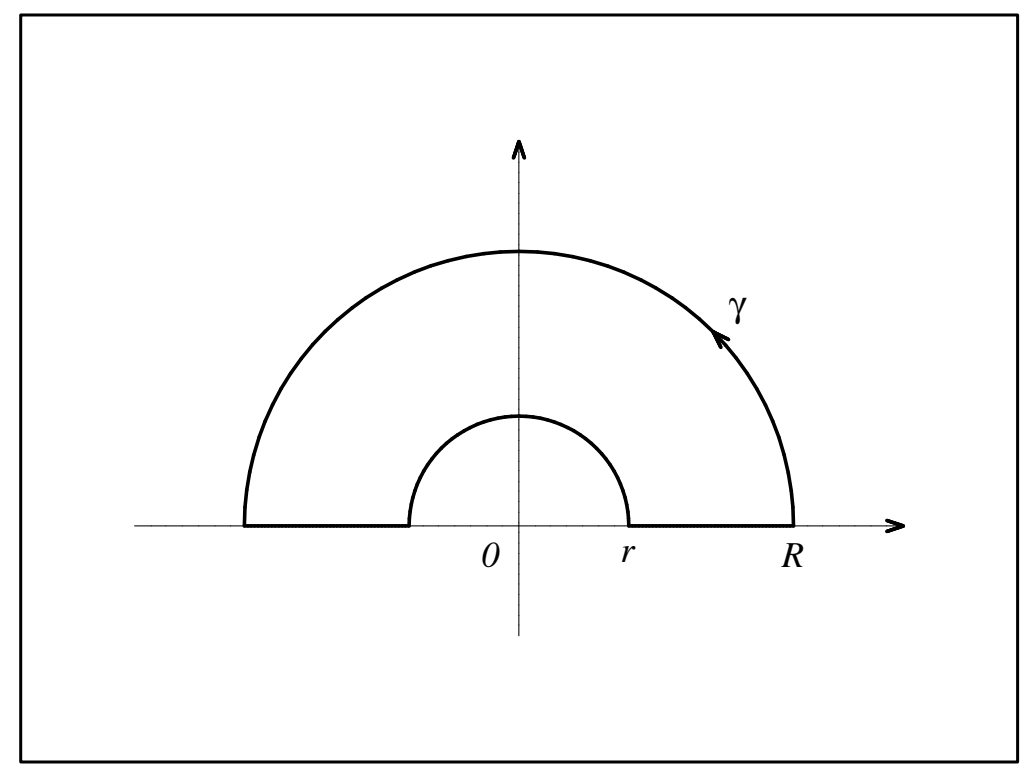

Figura 1.1:

$$
0=\int_{-R}^{-r} \frac{\mathbf{e}^{i t}}{t} d t+\int_{r}^{R} \frac{\mathbf{e}^{i t}}{t} d t+i \int_{\pi}^{0} \mathbf{e}^{i r \mathbf{e}^{i \theta}} d \theta+i \int_{0}^{\pi} \mathbf{e}^{i R \mathbf{e}^{i \theta}} d \theta
$$

Note que

$$
\left|\int_{0}^{\pi} \mathbf{e}^{i R \mathbf{e}^{i \theta}} d \theta\right| \leq \int_{0}^{\pi} \mathbf{e}^{-R \operatorname{sen} \theta} d \theta \rightarrow 0
$$

quando $R \rightarrow \infty$ e o resultado segue quando $r \rightarrow 0$.

(b) $\int_{-1}^{1} \frac{\operatorname{sen} N t}{\pi t} d t=\int_{-N}^{N} \frac{\operatorname{sen} t}{\pi t} d t \rightarrow 1$ quando $N \rightarrow \infty$ e

$$
\int_{-\infty}^{\infty} f(t) \frac{\operatorname{sen} N t}{\pi t} d t-f(0) \int_{-1}^{1} \frac{\operatorname{sen} N t}{\pi t} d t=\int_{|t| \leq 1} \frac{f(t)-f(0)}{\pi t} \operatorname{sen} N t d t+\int_{|t| \geq 1} \frac{f(t)}{\pi t} \operatorname{sen} N t d t
$$

ambos os termos a direita tendem a zero quando $N \rightarrow \infty$ pelo lema de Riemann-Lebesgue. 
Teorema 1.6.1 Suponha que A é o gerador de um semigrupo fortemente contínuo $\{T(t): t \geq$ $0\} \subset L(E)$ satisfazendo $\|T(t)\|_{L(E)} \leq M \mathbf{e}^{\beta t}$ e assuma que $\gamma>\max \{0, \beta\}$. Para qualquer $e \in D\left(A^{2}\right)$ e $t>0$

$$
T(t) e=\lim _{N \rightarrow \infty} \frac{1}{2 \pi i} \int_{\gamma-i N}^{\gamma+i N} \mathbf{e}^{\lambda t}(\lambda-A)^{-1} e d \lambda,
$$

onde a integral é ao longo do segmento de reta $\operatorname{com} \operatorname{Re} \lambda=\gamma$. O limite converge uniformemente para $\epsilon \leq t \leq 1 / \epsilon$, qualquer $\epsilon>0$.

Prova. Como $\operatorname{Re} \lambda=\gamma>\beta,(\lambda-A)^{-1}$ existe e é uniformemente limitada, de fato, como $e \in D\left(A^{2}\right)$

$$
(\lambda-A)^{-1} e=\lambda^{-1} e+\lambda^{-2} A e+\lambda^{-2}(\lambda-A)^{-1} A^{2} e
$$

então

$$
\begin{aligned}
\frac{1}{2 \pi i} \int_{\gamma-i N}^{\gamma+i N} \mathbf{e}^{\lambda t}(\lambda-A)^{-1} e d \lambda & =\frac{1}{2 \pi i} \int_{\gamma-i N}^{\gamma+i N} \frac{\mathbf{e}^{\lambda t}}{\lambda} e d \lambda \\
& +\frac{1}{2 \pi i} \int_{\gamma-i N}^{\gamma+i N} \frac{\mathbf{e}^{\lambda t}}{\lambda^{2}}\left[A e+(\lambda-A)^{-1} A^{2} e\right] d \lambda
\end{aligned}
$$

e ambos os termos convergem uniformemente em $\epsilon \leq t \leq 1 / \epsilon$ quando $N \rightarrow \infty$, o primeiro por

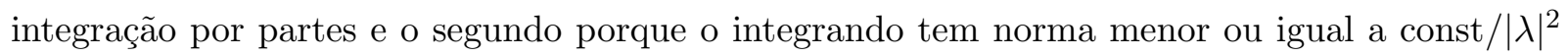
então converge absolutamente. Resta mostrar que o limite é $T(t) e$.

Agora para $\operatorname{Re} \lambda=\gamma$

$$
(\lambda-A)^{-1} e=\int_{0}^{\infty} \mathbf{e}^{-\lambda s} T(s) e d s
$$

então

$$
\begin{aligned}
\frac{1}{2 \pi i} \int_{\gamma-i N}^{\gamma+i N} \mathbf{e}^{\lambda t}(\lambda-A)^{-1} e d \lambda & =\int_{0}^{\infty}\left\{\frac{1}{2 \pi i} \int_{\gamma-i N}^{\gamma+i N} \mathbf{e}^{\lambda(t-s)} d \lambda\right\} T(s) e \\
& =\int_{0}^{\infty} \frac{\operatorname{sen} N(t-s)}{\pi(t-s)} \mathbf{e}^{\gamma(t-s)} T(s) e d s \\
& =\int_{-t}^{\infty} \frac{\operatorname{sen} N \tau}{\pi \tau} \mathbf{e}^{-\gamma \tau} T(t+\tau) e d \tau
\end{aligned}
$$

A função

$$
f(\tau)= \begin{cases}\left\langle e^{*}, T(t+\tau) e\right\rangle \mathbf{e}^{-\gamma \tau}, & \tau \geq-t \\ 0, & \tau<-t\end{cases}
$$

satisfaz as condições do lema para qualquer $e^{*} \in E^{*}$ e $t>0$ pois $f^{\prime}(0)=\left\langle e^{*}, T(t)(A-\gamma) e\right\rangle$. Então

$$
\left\langle e^{*}, \frac{1}{2 \pi i} \int_{\gamma-i N}^{\gamma+i N} \mathbf{e}^{\lambda t}(\lambda-A)^{-1} e d \lambda\right\rangle \longrightarrow f(0)=\left\langle e^{*}, T(t) e\right\rangle
$$

quando $N \rightarrow \infty$. Isto vale para todo $e^{*} \in E^{*}$ e a prova está completa. 


\subsection{Operadores Setoriais e Analiticidade}

Seja $A: D(A) \subset E \longrightarrow E$ o gerador de um semigrupo fortemente contínuo $\{T(t): t \geq 0\}$.

Definição 1.7.1 Se existem constantes a, $C$ e $\varphi \in(\pi / 2, \pi], \Sigma_{a, \varphi}=\{\lambda \in \mathbb{C}:|\arg (\lambda-a)|<\varphi\}$ está no conjunto resolvente de $A$ e

$$
\left\|(\lambda-A)^{-1}\right\|_{L(E)} \leq \frac{C}{|\lambda-a|} \quad \text { em } \Sigma_{a, \varphi}
$$

dizemos que $-A$ é setorial.

Teorema 1.7.1 Se $A: D(A) \subset E \longrightarrow E$ é um operador linear, tal que $\Sigma=\{\lambda \in \mathbb{C}:|\arg \lambda|<$ $\varphi\} \subset \rho(A)$ para algum $\varphi \in(\pi / 2, \pi)$ e que

$$
\left\|(\lambda-A)^{-1}\right\|_{L(E)} \leq \frac{C}{|\lambda|}, \quad \lambda \in \Sigma .
$$

Então o semigrupo gerado por A é analítico em um setor contendo o eixo real positivo.

Prova. Seja $e \in D\left(A^{2}\right), t>0$, então para algum $\gamma>0$

$$
T(t) e=\frac{1}{2 \pi i} \int_{\gamma-i \infty}^{\gamma+i \infty} \mathbf{e}^{\lambda t}(\lambda-A)^{-1} e d \lambda .
$$

O integrando é analítico para $\lambda \in \Sigma$ e portanto podemos deformar o contorno de integração para $\Gamma$, consistindo de dois raios $\{\lambda \in \mathbb{C}: \arg \lambda= \pm \phi,|\lambda|>r\}, \frac{\pi}{2}<\phi<\varphi$, e do arco de círculo $\{\lambda \in \mathbb{C}:|\lambda|=r,|\arg \lambda| \leq \phi\}$ para $r$ pequeno. Veja Figura 1.2. Note que, quando $\operatorname{Im} \lambda= \pm N$, $-k N \leq \operatorname{Re} \lambda \leq \gamma(k=|\cot \phi|>0)$,

$$
\left\|\mathbf{e}^{\lambda t}(\lambda-A)^{-1} e\right\|_{E} \leq \frac{\mathbf{e}^{t \operatorname{Re} \lambda} C\|e\|_{E}}{\sqrt{(\operatorname{Re} \lambda)^{2}+N^{2}}}
$$

e as integrais correspondentes tendem a zero quando $N \rightarrow \infty$.

Portanto

$$
T(t) e=\frac{1}{2 \pi i} \int_{\Gamma} \mathbf{e}^{\lambda t}(\lambda-A)^{-1} e d \lambda,
$$

e esta expressão vale para todo $e \in E$ porque converge em norma. De fato, para $t>0, \arg \lambda= \pm \phi$

$$
\left\|\mathbf{e}^{\lambda t}(\lambda-A)^{-1}\right\|_{L(E)} \leq C \frac{\mathbf{e}^{-t|\lambda| k_{1}}}{|\lambda|}, \quad k_{1}=|\cos \phi|>0
$$

então

$$
T(t)=\frac{1}{2 \pi i} \int_{\Gamma} \mathbf{e}^{\lambda t}(\lambda-A)^{-1} d \lambda
$$




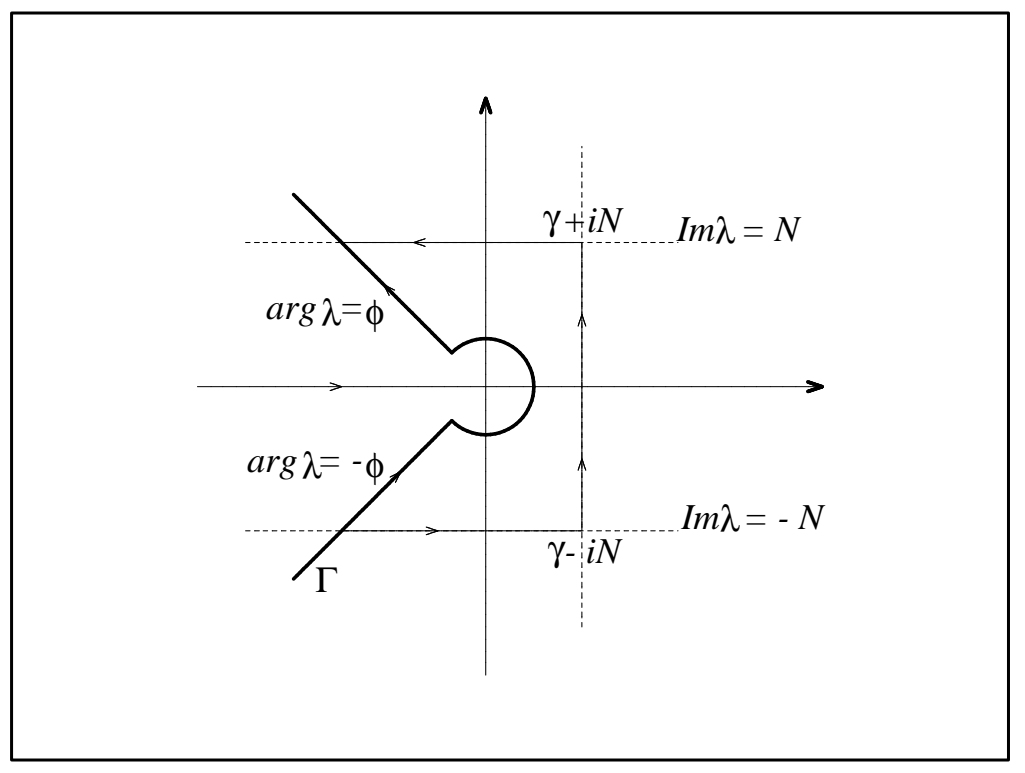

Figura 1.2:

com convergência na norma de $L(E)$ qualquer $t>0$. A convergência é uniforme para $\epsilon \leq t$, qualquer $\epsilon>0$, então $t \mapsto T(t) \in L(E)$ é contínuo para $t>0$ (mas claramente a convergência não é uniforme quando $t \rightarrow 0$, a menos que $A$ seja limitado). Ainda mais, a integral converge uniformemente para $t$ complexo em $|\arg t| \leq \epsilon_{1}<\phi-\pi / 2, \epsilon_{0} \leq|t|,\left(\epsilon_{i}>0, i=0,1\right)$, logo $t \mapsto T(t)$ é analítico em um setor $|\arg t|<\phi-\pi / 2$ contendo o eixo real positivo.

Esta prova de analiticidade não usa o fato que $A$ é o gerador de um semigrupo mas somente propriedades do resolvente $(\lambda-A)^{-1}$ quando $|\lambda| \rightarrow \infty$. De fato, qualquer operador densamente definido $A$ tal que $-A$ é setorial gera um semigrupo analítico.

Teorema 1.7.2 Assuma que $A: D(A) \subset E \rightarrow E$ é densamente definido $e-A$ é setorial. Então A é o gerador infinitesimal de um semigrupo fortemente contínuo $\{T(t): t \geq 0\} \subset L(E)$,

$$
T(t)=\frac{1}{2 \pi i} \int_{\Gamma_{a}} \mathbf{e}^{\lambda t}(\lambda-A)^{-1} d \lambda
$$

onde $\Gamma_{a}$ é a fronteira de $\Sigma_{a, \phi} \backslash\{\lambda \in \mathbb{C}:|\lambda-a| \leq r\}, \frac{\pi}{2}<\phi<\varphi, r$ pequeno, orientada no sentido da parte imaginária crescente. Adicionalmente, $t \mapsto T(t)$ se estende a uma função analítica de $\{t \in \mathbb{C}:|\arg t|<\phi-\pi / 2\}$ em $L(E)$ (ou a complexificação de $E$, se $E$ é um espaço de Banach real) e para algum $K>0$

$$
\|T(t)\|_{L(E)} \leq K \mathbf{e}^{a t}, \quad\|A T(t)\|_{L(E)} \leq K t^{-1} \mathbf{e}^{a t}
$$


para todo $t>0$. Note que

$$
\frac{d}{d t} T(t)=A T(t)
$$

é um operador limitado para qualquer $t>0$.

Prova. Não há perda de generalidade em assumir que $a=0$. Defina $T(t)$ por

$$
T(t)=\frac{1}{2 \pi i} \int_{\Gamma_{a}} \mathbf{e}^{\lambda t}(\lambda-A)^{-1} d \lambda
$$

tome $\lambda=a+\mu$, então

$$
\mathbf{e}^{-a t} T(t)=\frac{1}{2 \pi i} \int_{\Gamma_{0}} \mathbf{e}^{\mu t}(\mu-(A-a))^{-1} d \mu
$$

e $\|\left(\mu-(A-a)^{-1} \|_{L(E)} \leq C /|\mu|\right.$.

Como observado acima, $t \mapsto T(t)$ é analítica. Nós primeiro provamos que $\|T(t)\|_{L(E)}$ e $t\|A T(t)\|_{L(E)}$ são limitados para $t>0$. Mudando variáveis para $\mu=\lambda t$,

$$
T(t)=\frac{1}{2 \pi i} \int_{\Gamma_{0}} \mathbf{e}^{\mu}\left(\frac{\mu}{t}-A\right)^{-1} \frac{d \mu}{t},
$$

e o contorno é ainda $\Gamma_{0}$ já que o integrando é analítico. Logo

$$
\|T(t)\|_{L(E)} \leq \frac{1}{2 \pi} \int_{\Gamma_{0}} \mathbf{e}^{\operatorname{Re} \mu} \frac{C}{|\mu| / t} \frac{|d \mu|}{t}=K<\infty
$$

uniformemente para $t>0$. Semelhantemente

$$
\begin{aligned}
\frac{1}{2 \pi i} \int_{\Gamma_{0}} \mathbf{e}^{\lambda t} A(\lambda-A)^{-1} d \lambda & =\frac{1}{2 \pi i} \int_{\Gamma_{0}} \mathbf{e}^{\lambda t}\left[-I+\lambda(\lambda-A)^{-1}\right] d \lambda \\
& =-\frac{1}{2 \pi i} \int_{\Gamma_{0}} \mathbf{e}^{\lambda t} d \lambda+\frac{t^{-1}}{2 \pi i} \int_{\Gamma_{0}} \mathbf{e}^{\mu} \frac{\mu}{t}\left(\frac{\mu}{t}-A\right)^{-1} d \mu
\end{aligned}
$$

o primeiro termo é zero e o segundo é estimado da seguinte forma

$$
\left\|\frac{t^{-1}}{2 \pi i} \int_{\Gamma_{0}} \mathbf{e}^{\mu} \frac{\mu}{t}\left(\frac{\mu}{t}-A\right)^{-1} d \mu\right\|_{L(E)} \leq \frac{1}{2 \pi} \frac{1}{t} \int_{\Gamma_{0}} \mathbf{e}^{\operatorname{Re} \mu} C|d \mu|=K_{1} t^{-1}<\infty .
$$

Para ver que isto é $A T(t)$, note que $A$ é um operador fechado, pois $(\lambda-A)^{-1} \in L(E)$ para $\lambda \in \Sigma_{0}$. Como a integral que define $T(t)$ é um limite de somas de Riemann é fácil ver que $A T(t) e=T(t) A e$ para todo $e \in D(A)$.

Pela analiticidade e convergência uniforme para cada $t>0$, temos

$$
\frac{d}{d t} T(t)=\frac{1}{2 \pi i} \int_{\Gamma_{0}} \mathbf{e}^{\lambda t} \lambda(\lambda-A)^{-1} d \lambda,
$$

que é $A T(t)$ como mostrado acima. Seja $e \in D(A), t>0$ e

$$
T(t) e=\left(\frac{1}{2 \pi i} \int_{\Gamma_{0}} \mathbf{e}^{\lambda t} \frac{d \lambda}{\lambda}\right) e+\frac{t}{2 \pi i} \int_{\Gamma_{0}} \mathbf{e}^{\mu} \frac{\mu}{t}\left(\frac{\mu}{t}-A\right)^{-1} A e \frac{d \mu}{\mu^{2}}
$$


$\log 0$

$$
\|T(t) e-e\|_{E} \leq \frac{t}{2 \pi} \int_{\Gamma_{0}} \mathbf{e}^{\operatorname{Re} \mu} C\|A e\|_{E}\left|\frac{d \mu}{\mu^{2}}\right|=\mathcal{O}(t)
$$

quando $t \rightarrow 0^{+}$. Como $\|T(t)\|_{L(E)}$ é limitado quando $t \rightarrow 0^{+}, T(t) e \rightarrow e$ quando $t \rightarrow 0^{+}$para todo $e \in E$. Finalmente, para $0 \leq s \leq t$ a aplicação $s \mapsto T(t-s) T(s) e$ é contínua e é diferenciável (analítica) para $0<s<t$, com

$$
\frac{d}{d s}(T(t-s) T(s) e)=-A T(t-s) T(s) e+T(t-s) A T(s) e=0
$$

então é constante e

$$
T(t-s) T(s) e=T(t) e, \quad \text { para } \quad 0 \leq s \leq t, e \in E .
$$

Esta é a propriedade de semigrupo e a prova de que $T(t)$ é um semigrupo fortemente contínuo está completa. Para completar a prova do teorema, devemos mostrar que $A$ é seu gerador. Mas

$$
T(t) e-e=\int_{0}^{t} T(s) A e d s
$$

quando $t>0, e \in D(A)$, então $\frac{1}{t}(T(t) e-e) \rightarrow A e$ quando $t \rightarrow 0^{+}$e $A$ está contido no gerador. $A$ é de fato o gerador pois 1 está no resolvente de $A$ e do gerador. 


\section{Capítulo 2}

\section{Potências Fracionárias}

Neste capítulo iremos apresentar o conceito de potências fracionárias, bem como alguns resultados que caracterizam os espaços de potências fracionárias.

\subsection{Introdução}

Vamos começar esta seção motivando a definição de potências fracionárias de operadores fechados. Em primeiro lugar observe que se $\gamma$ é uma curva fechada, retificável e simples em $\mathbb{C} \backslash(-\infty, 0]$ e $n(\gamma ; a)$ denota o índice da curva $\gamma$ em $a \in \mathbb{C}$, temos do Teorema dos Resíduos que

$$
a^{\alpha}=\frac{1}{2 \pi i} \int_{\gamma} \frac{\lambda^{\alpha}}{\lambda-a} d \lambda
$$

para todo $\alpha \in \mathbb{C}$ e $a \in \mathbb{C} \operatorname{com} n(\gamma ; a)=1$. Aqui $\lambda^{\alpha}=\mathbf{e}^{\alpha \log \lambda}$ e $\log \lambda$ é o ramo principal do logarítimo.

Se $A \in L(E)$ é tal que $\sigma(A) \subset \mathbb{C} \backslash(-\infty, 0]$ e $\gamma$ é uma curva fechada, retificável e simples em $\mathbb{C} \backslash(-\infty, 0]$ tal que $n(\gamma ; a)=1, \forall a \in \sigma(A)$, definimos em analogia com a observação acima

$$
A^{\alpha}=\frac{1}{2 \pi i} \int_{\gamma} \lambda^{\alpha}(\lambda-A)^{-1} d \lambda
$$

para todo $\alpha \in \mathbb{C}$. É fácil ver, da expressão acima, que $I^{\alpha}=I$ para todo $\alpha \in \mathbb{C}$.

É claro que $A^{\alpha} \in L(E)$. Mostremos que $A^{\alpha} A^{\beta}=A^{\alpha+\beta}\left(\left\{A^{\alpha}, \alpha \in \mathbb{C}\right\}\right.$ é um grupo) e que $A^{n}$ coincide com a definição usual (a $n$-ésima iterada de $A$ ). Para mostrar a propriedade de grupo 
escolha $\gamma^{\prime}$ uma curva fechada, retificável e simples em $\mathbb{C} \backslash(-\infty, 0]$ externa a $\gamma$, então

$$
\begin{aligned}
A^{\alpha} A^{\beta} & =\frac{1}{2 \pi i} \int_{\gamma} \lambda^{\alpha}(\lambda-A)^{-1} d \lambda \frac{1}{2 \pi i} \int_{\gamma^{\prime}} \mu^{\beta}(\mu-A)^{-1} d \mu \\
& =\frac{1}{(2 \pi i)^{2}} \int_{\gamma^{\prime}} \int_{\gamma} \lambda^{\alpha} \mu^{\beta}(\lambda-A)^{-1}(\mu-A)^{-1} d \lambda d \mu \\
& =\frac{1}{(2 \pi i)^{2}} \int_{\gamma^{\prime}} \int_{\gamma} \lambda^{\alpha} \mu^{\beta} \frac{(\lambda-A)^{-1}-(\mu-A)^{-1}}{\mu-\lambda} d \lambda d \mu \\
& =\frac{1}{(2 \pi i)^{2}} \int_{\gamma} \lambda^{\alpha}(\lambda-A)^{-1} \int_{\gamma^{\prime}} \mu^{\beta} \frac{1}{\mu-\lambda} d \mu d \lambda \\
& +\frac{1}{(2 \pi i)^{2}} \int_{\gamma^{\prime}} \mu^{\beta}(\mu-A)^{-1} \int_{\gamma} \lambda^{\alpha} \frac{1}{\lambda-\mu} d \lambda d \mu \\
& =\frac{1}{2 \pi i} \int_{\gamma} \lambda^{\alpha+\beta}(\lambda-A)^{-1} d \lambda=A^{\alpha+\beta}
\end{aligned}
$$

onde na última passagem utilizamos o Teorema dos Resíduos para obter que

$$
\frac{1}{2 \pi i} \int_{\gamma^{\prime}} \mu^{\beta} \frac{1}{\mu-\lambda} d \mu=\lambda^{\beta}
$$

e observamos que $\mu$ está no traço de $\gamma^{\prime}$ que é externa a $\gamma$ e portanto, do Teorema de Cauchy,

$$
\int_{\gamma} \lambda^{\alpha} \frac{1}{\lambda-\mu} d \lambda=0
$$

Se por outro lado $\alpha=n$ é um número inteiro positivo podemos tomar $\gamma$ uma curva em $\mathbb{C}$ (não é necessário evitar o semi-eixo real negativo), já que $\lambda \mapsto \lambda^{n}$ é uma função inteira, temos então que

$$
A_{n}:=\frac{1}{2 \pi i} \int_{\gamma} \lambda^{n}(\lambda-A)^{-1} d \lambda=\frac{1}{2 \pi i} \int_{\gamma} \lambda^{n-1}\left(I-\lambda^{-1} A\right)^{-1} d \lambda
$$

Note que 0 é um polo de ordem $n$ do integrando e do Teorema dos Resíduos, obtemos que

$$
A_{n}=\frac{1}{2 \pi i} \int_{\gamma} \lambda^{n}(\lambda-A)^{-1} d \lambda=\operatorname{res}\left(\lambda^{n}(\lambda-A)^{-1}, 0\right) .
$$

Como para $|\lambda|>\|A\|$ temos que

$$
\left(I-\lambda^{-1} A\right)^{-1}=\sum_{j=0}^{\infty} \lambda^{-j} A^{j},
$$

e assim, obtemos a série de Laurent

$$
\lambda^{n-1}\left(I-\lambda^{-1} A\right)^{-1}=\sum_{j=0}^{\infty} \lambda^{(n-1)-j} A^{j} .
$$

Donde resulta que

$$
\operatorname{res}\left(\lambda^{n}(\lambda-A)^{-1}, 0\right)=A^{n}
$$


concluímos que

$$
A_{n}=A^{n}
$$

Ou seja, $A^{\alpha}$ é a $\alpha$ iterada de $A$ quando $\alpha \in \mathbb{N}$.

No que se segue buscaremos expressões equivalentes de $A^{\alpha}$ que façam sentido para uma classe mais ampla de operadores. Se $0<\phi<\pi$ e $A \in L(E)$ é tal que $\sigma(A) \subset \mathbb{C} \backslash(-\infty, 0]$. Seja $\Sigma_{\phi}=\{\lambda \in \mathbb{C}:|\arg \lambda|<\phi\}, B_{R}=\{\lambda \in \mathbb{C}:|\lambda|<R\}$ e $\Sigma_{R, \phi}=B_{R} \cap \Sigma_{\phi}$. Denote por $\Gamma_{R}$ a porção da fronteira de $\Sigma_{\phi}$ que está em $B_{R}$ orientada no sentido da parte imaginária decrescente, $\gamma_{R}$ a porção da fronteira de $B_{R}$ que está em $\Sigma_{\phi}$ orientada no sentido anti-horário. Com isto $\Gamma_{R}+\gamma_{R}$ é a fronteira de $\Sigma_{R, \phi}$. Escolha $R>2\|A\|$ e $\phi$ tal que $\Sigma_{R, \phi} \supset \sigma(A)$. Com isto temos que

$$
\begin{aligned}
A^{\alpha} & =\frac{1}{2 \pi i} \int_{\Gamma_{R}+\gamma_{R}} \lambda^{\alpha}(\lambda-A)^{-1} d \lambda \\
& =\frac{1}{2 \pi i} \int_{\Gamma_{R}} \lambda^{\alpha}(\lambda-A)^{-1} d \lambda+\frac{1}{2 \pi i} \int_{\gamma_{R}} \lambda^{\alpha}(\lambda-A)^{-1} d \lambda
\end{aligned}
$$

e, sobre $\gamma_{R}$,

$$
\left\|\lambda(\lambda-A)^{-1}\right\|=\left\|\left(I-\lambda^{-1} A\right)^{-1}\right\|=\left\|\sum_{n=0}^{\infty}\left(\frac{A}{\lambda}\right)^{n}\right\| \leq \frac{1}{1-\frac{\|A\|}{R}} \leq 2 .
$$

Se agora tomamos $\operatorname{Re} \alpha<0$, vamos mostrar que a integral sobre $\gamma_{R}$ em (2.2) converge para zero quando $R$ tende para infinito. De fato,

$$
\left\|\int_{\gamma_{R}} \lambda^{\alpha}(\lambda-A)^{-1} d \lambda\right\| \leq \int_{-\pi+\phi}^{\pi-\phi} R^{\operatorname{Re} \alpha} \mathbf{e}^{-\theta \operatorname{Im} \alpha}\left\|\left(R e^{i \theta}-A\right)^{-1}\right\| R d \theta
$$

e de (2.3) é fácil ver que a integral sobre $\gamma_{R}$ tende a zero quando $R$ tende para infinito.

Se $\Gamma$ denota a fronteira de $\Sigma_{\phi}$ orientada no sentido da parte imaginária decrescente, os cálculos acima mostram que sempre que $\operatorname{Re} \alpha<0$, temos

$$
A^{\alpha}=\frac{1}{2 \pi i} \int_{\Gamma} \lambda^{\alpha}(\lambda-A)^{-1} d \lambda
$$

Observe que a convergência da integral em (2.4) somente depende da estimativa espectral em (2.3) e não do operador $A$. Isto segue facilmente se parametrizarmos $\Gamma$. Vamos apenas considerar a parte $\Gamma_{+}$de $\Gamma$ com parte imaginária positiva. Então

$$
\left\|\int_{\Gamma_{+}} \lambda^{\alpha}(\lambda-A)^{-1} d \lambda\right\| \leq \int_{0}^{\infty} t^{\operatorname{Re} \alpha} \mathbf{e}^{-(\pi-\phi) \operatorname{Im} \alpha}\left\|\left(t \mathbf{e}^{i(\pi-\phi)}-A\right)^{-1}\right\| d t .
$$

Como o resolvente é contínuo sobre $\Gamma$ a convergência da integral acima segue somente de (2.3), ainda mais esta convergência é uniforme para $\alpha$ em qualquer compacto de $\{\lambda \in \mathbb{C}: \operatorname{Re} \lambda<0\}$. A convergência da integral sobre a parte de $\Gamma$ com parte real negativa segue de forma semelhante. 
Esta observação nos indica uma classe mais geral de operadores $A$ para os quais podemos definir as potências $A^{\alpha}$ com Re $\alpha<0$. Esta classe é a classe dos operadores fechados, densamente definidos $A$ com resolvente contendo um setor $\mathbb{C} \backslash \Sigma_{\phi} \cup\{0\}=-\Sigma_{\pi-\phi} \cup\{0\}$ e tais que $\lambda(\lambda-A)^{-1}$ é limitada em $-\Sigma_{\pi-\phi-\epsilon}, \pi-\phi>\epsilon>0,0<\phi<\pi$.

Note que se $\varphi=\pi-\phi$ então $\lambda(\lambda-A)^{-1}$ é limitado em $-\Sigma_{\varphi}$ se e somente se $\lambda(\lambda+A)^{-1}$ é limitado em $\Sigma_{\varphi}$ se e somente se $(1+|\lambda|)\left\|(\lambda+A)^{-1}\right\|$ é limitado em $\Sigma_{\varphi}$.

A seguir mostramos que se $(1+s)\left\|(s+A)^{-1}\right\| \leq M, s \in[0, \infty)$ então $(1+|\lambda|)\left\|(\lambda+A)^{-1}\right\|$ é limitado em $\Sigma_{\varphi}$ para $\varphi=\operatorname{arcsen} \frac{1}{2 M}$. Em particular, com isto teremos mostrado que podemos definir $A^{\alpha}$ através de (2.4) para todo operador $A$ tal que $-A$ gera um semigrupo fortemente contínuo $\{T(t): t \geq 0\}$ tal que $\|T(t)\| \leq M, t \geq 0$.

\subsection{Operadores do Tipo Positivo}

Seja $E$ um espaço de Banach. Um operador linear $A$ em $E$ é dito de tipo positivo com constante $M$ (veja [2]), $M \geq 1$ se é fechado, densamente definido, $\mathbb{R}^{+} \subset \rho(-A)$ e

$$
(1+s)\left\|(s+A)^{-1}\right\|_{L(E)} \leq M, \quad s \in \mathbb{R}^{+}
$$

Denotamos o conjunto dos operadores de tipo positivo por

$$
\mathcal{P}:=\mathcal{P}(E)
$$

Pelo restante desta seção assumimos que $A \in \mathcal{P}$.

Seja $A$ um operador de tipo positivo com constante $M$. Dado $s \in \mathbb{R}^{+}$e $\lambda \in \mathbb{C}$ satisfazendo

$$
|\lambda-s| \leq(1+s) /(2 M)
$$

segue de $\lambda+A=(s+A)\left(1+(\lambda-s)(s+A)^{-1}\right)$ que $\lambda \in \rho(-A)$ e

$$
\begin{aligned}
\left\|(\lambda+A)^{-1}\right\|_{L(E)} & \leq\left\|\left[1+(\lambda-s)(s+A)^{-1}\right]^{-1}\right\|_{L(E)}\left\|(s+A)^{-1}\right\|_{L(E)} \\
& \leq\left\|1+(\lambda-s)(s+A)^{-1}\right\|^{-1} \frac{M}{1+s} \leq 2 M(1+s)^{-1} \\
& \leq \frac{2 M}{1+|\lambda|} \frac{1+s+|\lambda-s|}{1+s} \leq \frac{2 M}{1+|\lambda|}\left(1+\frac{1}{2 M}\right)=\frac{2 M+1}{1+|\lambda|} .
\end{aligned}
$$

Na Figura 2.1, $\theta \in\left(0, \operatorname{arcsen} \frac{1}{2 M}\right]$ e deduzimos que

$$
\Sigma_{M}:=\{z \in \mathbb{C}:|\arg z| \leq \operatorname{arcsen} 1 /(2 M)\}+\{z \in \mathbb{C}:|z| \leq 1 /(2 M)\} \subset \rho(-A)
$$




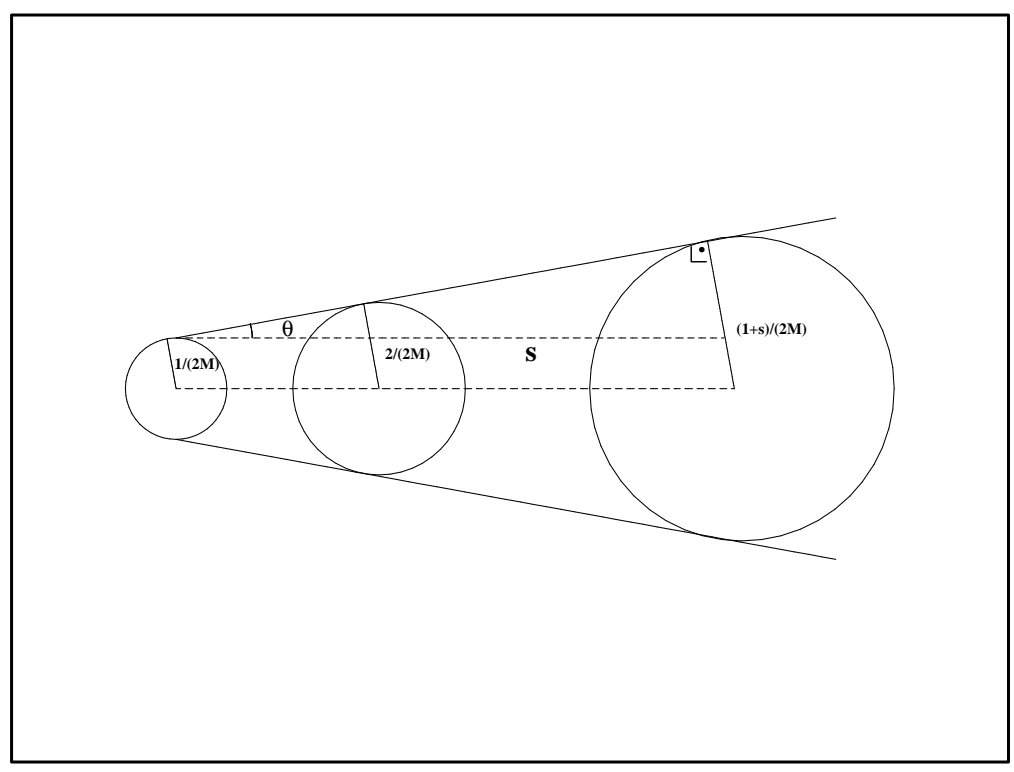

Figura 2.1:

e que

$$
(1+|\lambda|)\left\|(\lambda+A)^{-1}\right\|_{L(E)} \leq 2 M+1, \quad \lambda \in \Sigma_{M}
$$

Com isto para todo $A \in \mathcal{P}(E)$ e $\alpha \in \mathbb{C}$, com $\operatorname{Re} \alpha<0$, definimos

$$
A^{\alpha}:=\frac{1}{2 \pi i} \int_{\Gamma}(-\lambda)^{\alpha}(\lambda+A)^{-1} d \lambda=-\frac{1}{2 \pi i} \int_{\tilde{\Gamma}} \lambda^{\alpha}(\lambda-A)^{-1} d \lambda
$$

onde $\Gamma$ é qualquer curva simples em $\Sigma_{M} \backslash \mathbb{R}^{+}$suave por partes indo de $\infty \mathbf{e}^{-i \nu}$ até $\infty \mathbf{e}^{i \nu}$ para algum $\nu \in(0, \operatorname{arcsen}(1 / 2 M)]$. É claro que $-\Gamma:=\{\lambda \in \mathbb{C}:-\lambda \in \Gamma\}$ e $\tilde{\Gamma}$ é uma curva com o mesmo traço de $-\Gamma$, mas percorrida em sentido contrário. Mostremos que a integral acima é convergente. De fato, para $r<\frac{1}{2 M}$ e $\nu \in\left(0, \operatorname{arcsen} \frac{1}{2 M}\right]$, temos

$$
\begin{aligned}
\frac{1}{2 \pi i} \int_{\Gamma}(-\lambda)^{\alpha}(\lambda+A)^{-1} d \lambda & =\frac{1}{2 \pi i} \int_{r}^{\infty}\left(-s \mathbf{e}^{i \nu}\right)^{\alpha}\left(s \mathbf{e}^{i \nu}+A\right)^{-1} \mathbf{e}^{i \nu} d s \\
& +\frac{1}{2 \pi i} \int_{\infty}^{r}\left(-s \mathbf{e}^{-i \nu}\right)^{\alpha}\left(s \mathbf{e}^{-i \nu}+A\right)^{-1} \mathbf{e}^{-i \nu} d s \\
& +\frac{1}{2 \pi i} \int_{2 \pi-\nu}^{\nu}\left(-r \mathbf{e}^{i \varphi}\right)^{\alpha}\left(r \mathbf{e}^{i \varphi}+A\right)^{-1} r i \mathbf{e}^{i \varphi} d \varphi
\end{aligned}
$$

e, observe ainda que se $\alpha \in \mathbb{C}$, então

$$
\left|\lambda^{\alpha}\right|=\left|\mathbf{e}^{\ln \lambda^{\alpha}}\right|=\left|\mathbf{e}^{(\operatorname{Re} \alpha+i \operatorname{Im} \alpha)(\ln |\lambda|+i \arg \lambda)}\right|=\mathbf{e}^{\operatorname{Re} \alpha \ln |\lambda|} \mathbf{e}^{-\operatorname{Im} \alpha \arg \lambda} \leq c|\lambda|^{\operatorname{Re} \alpha},
$$


com isso, temos

$$
\begin{aligned}
\left\|\int_{r}^{\infty}\left(-s \mathbf{e}^{i \nu}\right)^{\alpha}\left(s \mathbf{e}^{i \nu}+A\right)^{-1} \mathbf{e}^{i \nu} d s\right\| & \leq \int_{r}^{\infty} c\left|-s \mathbf{e}^{i \nu}\right|^{\operatorname{Re} \alpha}\left\|\left(s \mathbf{e}^{i \nu}+A\right)^{-1}\right\| d s \\
& \leq c(2 M+1) \int_{r}^{\infty} s^{\operatorname{Re} \alpha} \frac{1}{1+s} d s \\
& \leq c(2 M+1) \int_{r}^{\infty} s^{\operatorname{Re} \alpha-1} d s<\infty
\end{aligned}
$$

Ainda,

$$
\begin{aligned}
\left\|\int_{\infty}^{r}\left(-s \mathbf{e}^{-i \nu}\right)^{\alpha}\left(s \mathbf{e}^{-i \nu}+A\right)^{-1} \mathbf{e}^{-i \nu} d s\right\| & \leq \int_{r}^{\infty} c\left|-s \mathbf{e}^{-i \nu}\right|^{\operatorname{Re} \alpha}\left\|\left(s \mathbf{e}^{-i \nu}+A\right)^{-1}\right\| d s \\
& \leq c(2 M+1) \int_{r}^{\infty} s^{\operatorname{Re} \alpha} \frac{1}{1+s} d s \\
& \leq c(2 M+1) \int_{r}^{\infty} s^{\operatorname{Re} \alpha-1} d s<\infty,
\end{aligned}
$$

e

$$
\begin{aligned}
\left\|\int_{2 \pi-\nu}^{\nu}\left(-r \mathbf{e}^{i \varphi}\right)^{\alpha}\left(r \mathbf{e}^{i \varphi}+A\right)^{-1} r i \mathbf{e}^{i \varphi} d \varphi\right\| & \leq \int_{\nu}^{2 \pi-\nu} c r^{\operatorname{Re} \alpha}\left\|\left(r \mathbf{e}^{i \varphi}+A\right)^{-1}\right\| r d \varphi \\
& \leq c(2 M+1) \int_{\nu}^{2 \pi-\nu} \frac{r^{\operatorname{Re} \alpha+1}}{1+r} d \varphi \\
& \leq 2 c(2 M+1)(\pi-\nu) r^{\operatorname{Re} \alpha+1}<\infty .
\end{aligned}
$$

Segue que $A^{\alpha}$ está bem definido em $L(E)$ e independe da escolha de $\Gamma$. Ainda, mais é verdade.

Lema 2.2.1 Para todo $\alpha$ e $\beta$ com parte real negativa $A^{\alpha} A^{\beta}=A^{\alpha+\beta}$.

Prova. Dados $\alpha$ e $\beta$ com $\operatorname{Re} \alpha<0$ e $\operatorname{Re} \beta<0$, escolha $\Gamma_{1}$ e $\Gamma_{2}$ como em (2.7), de forma que $\Gamma_{1}$ fique a esquerda de $\Gamma_{2}$. Então

$$
\begin{aligned}
A^{\alpha} A^{\beta} & =\frac{1}{(2 \pi i)^{2}} \int_{\Gamma_{1}} \int_{\Gamma_{2}}(-\lambda)^{\alpha}(-\mu)^{\beta}(\lambda+A)^{-1}(\mu+A)^{-1} d \mu d \lambda \\
& =\frac{1}{(2 \pi i)^{2}} \int_{\Gamma_{1}} \int_{\Gamma_{2}}(-\lambda)^{\alpha}(-\mu)^{\beta}(\lambda-\mu)^{-1}\left[(\mu+A)^{-1}-(\lambda+A)^{-1}\right] d \mu d \lambda \\
& =\frac{1}{2 \pi i} \int_{\Gamma_{2}}(-\mu)^{\beta}(\mu+A)^{-1}\left(\frac{1}{2 \pi i} \int_{\Gamma_{1}} \frac{(-\lambda)^{\alpha}}{\lambda-\mu} d \lambda\right) d \mu \\
& +\frac{1}{2 \pi i} \int_{\Gamma_{1}}(-\lambda)^{\alpha}(\lambda+A)^{-1}\left(\frac{1}{2 \pi i} \int_{\Gamma_{2}} \frac{(-\mu)^{\beta}}{\mu-\lambda} d \mu\right) d \lambda .
\end{aligned}
$$

Para cada $\mu \in \Gamma_{2}$, a aplicação $\lambda \mapsto(\lambda-\mu)^{-1}(-\lambda)^{\alpha}$ é analítica sobre $\Gamma_{1}$ e a esquerda dela. Portanto, segue de (2.7) e do Teorema de Cauchy que a integral no primeiro parêntesis é zero e a no segundo é igual a $(-\lambda)^{\beta}$. Conseqüêntemente,

$$
A^{\alpha} A^{\beta}=\frac{1}{2 \pi i} \int_{\Gamma_{1}}(-\lambda)^{\alpha+\beta}(\lambda+A)^{-1} d \lambda=A^{\alpha+\beta},
$$


o que prova a afirmativa.

Suponha que $0<\operatorname{Re} z<1$. Sabemos que

$$
A^{-z}=-\frac{1}{2 \pi i} \int_{\tilde{\Gamma}} \lambda^{-z}(\lambda-A)^{-1} d \lambda
$$

onde podemos escolher $\tilde{\Gamma} \subset \mathbb{C} / \mathbb{R}^{-}$, consistindo de:

$$
\left\{-s \mathbf{e}^{-i(\pi-\epsilon)}: s \in(-\infty,-r]\right\},\left\{r \mathbf{e}^{i \varphi}:|\varphi| \leq \pi-\epsilon\right\},\left\{s \mathbf{e}^{i(\pi-\epsilon)}: s \in[r, \infty)\right\}
$$

com $\epsilon>0$ e $r>0$ suficientemente pequenos. Segue que

$$
\begin{aligned}
A^{-z} & =-\frac{1}{2 \pi i} \int_{-\infty}^{-r}\left(-s \mathbf{e}^{-i(\pi-\epsilon)}\right)^{-z}\left(-s \mathbf{e}^{-i(\pi-\epsilon)}-A\right)^{-1}\left(-\mathbf{e}^{-i(\pi-\epsilon)}\right) d s \\
& -\frac{1}{2 \pi i} \int_{-(\pi-\epsilon)}^{\pi-\epsilon}\left(r \mathbf{e}^{i \varphi}\right)^{-z}\left(r \mathbf{e}^{i \varphi}-A\right)^{-1} r i \mathbf{e}^{i \varphi} d \varphi \\
& -\frac{1}{2 \pi i} \int_{r}^{\infty}\left(s \mathbf{e}^{i(\pi-\epsilon)}\right)^{-z}\left(s \mathbf{e}^{i(\pi-\epsilon)}-A\right)^{-1} \mathbf{e}^{i(\pi-\epsilon)} d s .
\end{aligned}
$$

Observe que dado $r>0$, temos

$$
\mathbf{e}^{-z(\ln |s|-i(\pi-\epsilon))}\left(-s \mathbf{e}^{-i(\pi-\epsilon)}-A\right)^{-1}\left(-\mathbf{e}^{-i(\pi-\epsilon)}\right) \stackrel{\epsilon \rightarrow 0}{\longrightarrow}(-s)^{-z} \mathbf{e}^{i \pi z}(s-A)^{-1},
$$

e

$$
\begin{aligned}
\left\|\mathbf{e}^{-z(\ln |s|-i(\pi-\epsilon))}\left(-s \mathbf{e}^{-i(\pi-\epsilon)}-A\right)^{-1}\left(-\mathbf{e}^{-i(\pi-\epsilon)}\right)\right\| & \leq \mathbf{e}^{-\operatorname{Re} z \ln (-s)-\operatorname{Im} z(\pi-\epsilon)} \frac{2 M+1}{1-s} \\
& \leq(2 M+1) \mathbf{e}^{|\operatorname{Im} z| \pi}(-s)^{-\operatorname{Re} z-1}
\end{aligned}
$$

onde a função dominante é integrável sobre $(-\infty,-r)$, pois $0<\operatorname{Re} z<1$. Então, usando o Teorema da Convergência Dominada, segue que

$$
\lim _{\epsilon \rightarrow 0} \int_{-\infty}^{-r} \mathbf{e}^{-z(\ln |s|-i(\pi-\epsilon))}\left(-s \mathbf{e}^{-i(\pi-\epsilon)}-A\right)^{-1}\left(-\mathbf{e}^{-i(\pi-\epsilon)}\right) d s=\int_{-\infty}^{-r}(-s)^{-z} \mathbf{e}^{i \pi z}(s-A)^{-1} d s
$$

Analogamente, para $r>0$,

$$
\mathbf{e}^{-z(\ln s+i(\pi-\epsilon))}\left(s \mathbf{e}^{i(\pi-\epsilon)}-A\right)^{-1} \mathbf{e}^{i(\pi-\epsilon)} \stackrel{\epsilon \rightarrow 0}{\longrightarrow}-s^{-z} \mathbf{e}^{-i \pi z}(-s-A)^{-1}
$$

e

$$
\begin{aligned}
\left\|\mathbf{e}^{-z(\ln s+i(\pi-\epsilon))}\left(s \mathbf{e}^{i(\pi-\epsilon)}-A\right)^{-1} \mathbf{e}^{i(\pi-\epsilon)}\right\| & \leq \mathbf{e}^{-\operatorname{Re} z \ln s+\operatorname{Im} z(\pi-\epsilon)} \frac{2 M+1}{1+s} \\
& \leq(2 M+1) \mathbf{e}^{|\operatorname{Im} z| \pi} s^{-\operatorname{Re} z-1}
\end{aligned}
$$

onde a função dominante é integrável sobre $(r, \infty)$, pois $0<\operatorname{Re} z<1$. Então, do Teorema da Convergência Dominada

$$
\int_{r}^{\infty} \mathbf{e}^{-z(\ln s+i(\pi-\epsilon))}\left(s \mathbf{e}^{i(\pi-\epsilon)}-A\right)^{-1} \mathbf{e}^{i(\pi-\epsilon)} d s \stackrel{\epsilon \rightarrow 0}{\longrightarrow} \int_{r}^{\infty}-s^{-z} \mathbf{e}^{-i \pi z}(-s-A)^{-1} d s
$$


Ainda, como

$$
\int_{-\pi}^{\pi}\left\|\mathbf{e}^{-z(\ln r+i \varphi)}\left(r \mathbf{e}^{i \varphi}-A\right)^{-1} r i \mathbf{e}^{i \varphi}\right\| d \varphi \leq \frac{2 M+1}{1+r} r^{1-\operatorname{Re} z} \int_{-\pi}^{\pi} \mathbf{e}^{\varphi \operatorname{Im} z} d \varphi<\infty,
$$

obtemos que

$$
\lim _{\epsilon \rightarrow 0} \int_{-(\pi-\epsilon)}^{\pi-\epsilon} \mathbf{e}^{-z(\ln r+i \varphi)}\left(r \mathbf{e}^{i \varphi}-A\right)^{-1} r i \mathbf{e}^{i \varphi} d \varphi=\int_{-\pi}^{\pi} \mathbf{e}^{-z(\ln r+i \varphi)}\left(r \mathbf{e}^{i \varphi}-A\right)^{-1} r i \mathbf{e}^{i \varphi} d \varphi
$$

Então, obtemos de (2.8), (2.9) e (2.10) que

$$
\begin{aligned}
A^{-z} & =\frac{1}{2 \pi i} \int_{-\infty}^{-r}(-s)^{-z} \mathbf{e}^{i \pi z}(s-A)^{-1} d s \\
& -\frac{1}{2 \pi i} \int_{-\pi}^{\pi} \mathbf{e}^{-z(\ln r+i \varphi)}\left(r \mathbf{e}^{i \varphi}-A\right)^{-1} r i \mathbf{e}^{i \varphi} d \varphi \\
& -\frac{1}{2 \pi i} \int_{r}^{\infty} s^{-z} \mathbf{e}^{-i \pi z}(s+A)^{-1} d s
\end{aligned}
$$

Ainda,

$$
\left\|\mathbf{e}^{-z(\ln r+i \varphi)}\left(r \mathbf{e}^{i \varphi}-A\right)^{-1} r i \mathbf{e}^{i \varphi}\right\| \leq \frac{2 M+1}{1+r} r^{1-\operatorname{Re} z} \mathbf{e}^{\operatorname{Im} z \varphi} \stackrel{r \rightarrow 0}{\longrightarrow} 0,
$$

pois, $\operatorname{Re} z>0$. Então, do Teorema da Convergência Dominada, resulta que

$$
\begin{aligned}
A^{-z} & =\frac{1}{2 \pi i} \int_{0}^{\infty} s^{-z} \mathbf{e}^{i \pi z}(s+A)^{-1} d s-\frac{1}{2 \pi i} \int_{0}^{\infty} s^{-z} \mathbf{e}^{-i \pi z}(s+A)^{-1} d s \\
& =\frac{1}{2 \pi i}\left(\mathbf{e}^{i \pi z}-\mathbf{e}^{-i \pi z}\right) \int_{0}^{\infty} s^{-z}(s+A)^{-1} d s
\end{aligned}
$$

isto é,

$$
A^{-z}=\frac{\operatorname{sen} \pi z}{\pi} \int_{0}^{\infty} s^{-z}(s+A)^{-1} d s, \quad 0<\operatorname{Re} z<1 .
$$

Aplicando a fórmula (2.11) ao caso $E:=\mathbb{C}$ e $A:=1$, em particular, segue que

$$
\int_{0}^{\infty} s^{-z}(1+s)^{-1} d s=\frac{\pi}{\operatorname{sen} \pi z}, \quad 0<\operatorname{Re} z<1 .
$$

Portanto deduzimos do fato que $A \in \mathcal{P}$ e da igualdade acima que

$$
\left\|A^{-z}\right\|_{L(E)} \leq M \frac{|\operatorname{sen} \pi z|}{\pi} \int_{0}^{\infty} s^{-\operatorname{Re} z}(1+s)^{-1} d s=M \frac{|\operatorname{sen} \pi z|}{\operatorname{sen} \pi \operatorname{Re} z}
$$

para $0<\operatorname{Re} z<1$. Agora não é difícil provar o seguinte resultado de continuidade:

Teorema 2.2.1 $\left\{A^{z}: \operatorname{Re} z<0\right\} \cup\left\{A^{0}=I_{E}\right\}$ é um semigrupo fortemente contínuo e analítico sobre E.

Prova. É uma conseqüência simples do teorema da derivação sob o sinal de integração que a aplicação $z \mapsto A^{z}$ é analítica em $\{z \in \mathbb{C}: \operatorname{Re} z<0\}$. Portanto, graças ao Lema 2.2.1, resta mostrar que o semigrupo é fortemente contínuo em $z=0$. 
Note que

$$
(s+A)^{-1}-(1+s)^{-1}=(s+A)^{-1}\left(I-(1+s)^{-1}(s+A)\right)=(1+s)^{-1}(s+A)^{-1}(I-A)
$$

para $s>0$. Portanto, dado $e \in D(A)$ e $z \in \mathbb{C}$ com $0<\operatorname{Re} z<1$, segue de (2.11) e de (2.12) que

$$
\begin{aligned}
A^{-z} e-e & =\frac{\operatorname{sen} \pi z}{\pi} \int_{0}^{\infty} s^{-z}(s+A)^{-1} e d s-\frac{\operatorname{sen} \pi z}{\pi} \int_{0}^{\infty} s^{-z}(1+s)^{-1} e d s \\
& =\frac{\operatorname{sen} \pi z}{\pi} \int_{0}^{\infty} \frac{s^{-z}}{1+s}(s+A)^{-1}(1-A) e d s
\end{aligned}
$$

Conseqüêntemente,

$$
\left\|A^{-z} e-e\right\|_{E} \leq M \frac{|\operatorname{sen} \pi z|}{\pi}\|(1-A) e\|_{E} \int_{0}^{\infty} \frac{s^{-\operatorname{Re} z}}{(1+s)^{2}} d s, \quad 0<\operatorname{Re} z<1 .
$$

Como a integral converge para 1 quando $\operatorname{Re} z \rightarrow 0$, vemos que $A^{-z} e \rightarrow e$ quando $z \rightarrow 0$ em $\{z \in \mathbb{C}:|\arg z| \leq \alpha\}$ para cada $\alpha \in(0, \pi / 2)$. Desde que $A^{-z}$ é uniformemente limitado para $z \in\{z \in \mathbb{C}:|\arg z| \leq \alpha\} \cap\{z \in \mathbb{C}: 0<\operatorname{Re} z<1\}$ para cada $\alpha \in(0, \pi / 2)$, graças a $(2.12), A^{z}$ converge para $I_{E}$ na topologia forte quando $z \rightarrow 0$ em $\{z \in \mathbb{C}:|\arg z| \geq \pi / 2+\epsilon\}$ para cada $\epsilon \in(0, \pi / 2)$. Isto prova o teorema.

Lema 2.2.2 Seja $A: D(A) \subset E \longrightarrow E$ um operador positivo. Então, $A^{z}$ é injetiva.

Prova. Fixe $z \in \mathbb{C}$ tal que $\operatorname{Re} z<0$. Suponha que $A^{z} e=0$ para algum $e \in E$ e $z \in \mathbb{C}$ com $\operatorname{Re} z<0$. Então segue que $A^{z+w} e=A^{w} A^{z} e=0$ para $\operatorname{Rew}<0$. Portanto $A^{w} e=0$ para $\operatorname{Re} w<\operatorname{Re} z$. Em particular, $A^{-k} e=0$ para $k=0,1,2, \cdots \operatorname{com} k>-\operatorname{Re} z$. Conseqüêntemente, $e=0$. Isto mostra que $A^{z}$ é injetiva para $\operatorname{Re} z<0$.

O Lema 2.2.2, nos diz que podemos definir as potências fracionárias de operadores positivos com expoente complexo com parte real positiva.

Definição 2.2.1 Seja $A: D(A) \subset E \longrightarrow E$ um operador positivo. Definimos

$$
A^{z}:=\left(A^{-z}\right)^{-1}
$$

para $z \in \mathbb{C} \operatorname{com} \operatorname{Re} z>0$.

Denotaremos por $C(E):=C(E, E)$ o conjunto dos operadores lineares fechados de $E$ em $E$. Claramente, $A^{z} \in \mathcal{C}(E)$. Dados $z, w \in \mathbb{C}$ com $0<\operatorname{Re} z<\operatorname{Re} w$ e $e \in D\left(A^{w}\right)$, segue de

$$
e=A^{-w} A^{w} e=A^{-z-(w-z)} A^{w} e
$$


que $e \in D\left(A^{z}\right)$, isto é,

$$
D\left(A^{w}\right) \subset D\left(A^{z}\right), \quad 0<\operatorname{Re} z<\operatorname{Re} w .
$$

Dado $e \in D(A)$, faça $f:=A e$. Como $D(A)$ é denso em $E$, podemos encontrar para cada $\epsilon>0$ um elemento $u \in D(A)$ tal que $\|u-f\|_{E} \leq \epsilon /\left\|A^{-1}\right\|_{L(E)}$. Portanto, fazendo $v:=A u$,

$$
\left\|A^{-2} v-e\right\|_{E}=\left\|A^{-1} u-A^{-1} f\right\|_{E} \leq\left\|A^{-1}\right\|_{L(E)}\|u-f\|_{E} \leq \epsilon .
$$

Isto mostra que $\overline{D\left(A^{2}\right)} \supset D(A)$. Portanto $\overline{D\left(A^{2}\right)} \supset \overline{D(A)}=E$ o que garante que $D\left(A^{2}\right)$ é denso em $E$. Por indução vemos que $D\left(A^{k}\right)$ é denso em $E$ para $k=1,2,3, \cdots$. Segue de $(2.14)$ que

$$
\overline{D\left(A^{z}\right)}=E, \quad \operatorname{Re} z>0
$$

Agora suponha que $\operatorname{Re} z>0$ e $\operatorname{Re} w>0$. Dado

$$
e \in D\left(A^{z+w}\right) \subset D\left(A^{w}\right) \cap D\left(A^{z}\right),
$$

faça $f:=A^{z+w} e$. Então $e=A^{-(z+w)} f=A^{-w} A^{-z} f \Rightarrow A^{w} e=A^{-z} f$ o que, por sua vez, mostra que $f=A^{z} A^{w} e$; isto é,

$$
A^{z+w} e=A^{z} A^{w} e=A^{w} A^{z} e, \quad e \in D\left(A^{z+w}\right) .
$$

Se $\operatorname{Re} z>\operatorname{Rew}$ e $e \in D\left(A^{w}\right)$ então

$$
A^{-z} A^{w} e=A^{-(z-w)} A^{-w} A^{w} e=A^{-(z-w)} e=A^{w-z} e .
$$

Adicionalmente, se $e \in D\left(A^{z}\right)$ então, graças a identidade acima,

$$
A^{-w} A^{z} e=A^{-w} A^{w} A^{z-w} e=A^{z-w} e .
$$

Isto prova que, dados $z, w \in \mathbb{C}$ com $\operatorname{Re} z, \operatorname{Re} w, \operatorname{Re}(z+w) \neq 0$,

$$
A^{z} A^{w} e=A^{z+w} e, \quad e \in D\left(A^{u}\right)
$$

onde $u \in\{z, w, z+w\}$ com $\operatorname{Re} u=\max \{\operatorname{Re} z, \operatorname{Re} w, \operatorname{Re}(z+w)\}$.

Considere a seguinte extensão de (2.11).

Proposição 2.2.1 Suponha que $m=0,1,2, \cdots$. Então

$$
A^{-z}=\frac{\operatorname{sen} \pi z}{\pi} \frac{m !}{(1-z)(2-z) \cdots(m-z)} \int_{0}^{\infty} s^{m-z}(s+A)^{-m-1} d s
$$

para $0<\operatorname{Re} z<m+1$. 
Prova. Suponha que $z$ satisfaz $0<\operatorname{Re} z<1$. Então da integração por partes em (2.11) temos que,

$$
\begin{aligned}
A^{-z} & =\frac{\operatorname{sen} \pi z}{\pi(1-z)}\left[\left.s^{1-z}(s+A)^{-1}\right|_{0} ^{\infty}+\int_{0}^{\infty} s^{1-z}(s+A)^{-2} d s\right] \\
& =\frac{\operatorname{sen} \pi z}{\pi(1-z)} \int_{0}^{\infty} s^{1-z}(s+A)^{-2} d s .
\end{aligned}
$$

Agora (2.17) segue por indução para $0<\operatorname{Re} z<1$. Graças a (2.5) é fácil verificar que a integral em (2.17) converge absolutamente para $0<\operatorname{Re} z<m+1$ e que o lado direito de (2.17) é uma aplicação analítica de $\{z \in \mathbb{C}: 0<\operatorname{Re} z<m+1\}$ em $L(E)$. Agora a afirmativa segue do Teorema 2.2 .1 .

É uma conseqüência do Teorema 2.2.1 que $\left\{A^{-t}: t \geq 0\right\}$ é um semigrupo fortemente contínuo sobre $E$. Denotamos o seu gerador infinitesimal por

$$
-\log A
$$

o que define o logarítimo de $A \in \mathcal{P}(E)$. Então a fórmula intuitiva

$$
A^{-t}=\mathbf{e}^{-t \log A}, \quad t \geq 0,
$$

é válida.

Um semigrupo analítico $\left\{\mathbf{e}^{-t B}: t \geq 0\right\}$ é dito de ângulo $\alpha$, onde $0<\alpha \leq \pi$, se existe uma função analítica

$$
T:\{z \in \mathbb{C}:|\arg z|<\alpha\} \rightarrow L(E)
$$

estendendo $\left\{\mathbf{e}^{-t B}: t \geq 0\right\}$, tal que $T$ é fortemente contínuo no setor fechado $\{z \in \mathbb{C}:|\arg z| \leq$ $\alpha-\epsilon\} \cup\{0\}$ para cada $\epsilon \in(0, \alpha)$. Então $T$ é um semigrupo sobre $E$ e escrevemos $\mathbf{e}^{-z B}:=T(z)$ para $\{z \in \mathbb{C}:|\arg z|<\alpha\} \cup\{0\}$. Esta notação é justificada já que $\frac{d}{d z} T(z)=-B T(z)$ para $z \in\{z \in \mathbb{C}:|\arg z|<\alpha\}$.

Teorema 2.2.2 Assuma que $A \in \mathcal{P}_{M}(E)$. Então $\left\{A^{-t}: t \geq 0\right\}$ é um semigrupo analítico de ângulo $\pi / 2$. Adicionalmente,

$$
\left\|A^{-t}\right\|_{L(E)} \leq M^{m}, \quad 0 \leq t \leq m, \quad m=0,1,2, \cdots,
$$

e $A^{-z}=\mathbf{e}^{-z \log A}$ para $\operatorname{Re} z<0$.

Prova. Graças ao Teorema 2.2.1 resta provar apenas a limitação. Aplicando a Proposição 2.2.1 a $E:=\mathbb{C}$ e $A:=1$ vemos que

$$
\frac{\pi(1-t)(2-t) \cdots(m-t)}{m ! \operatorname{sen} \pi t}=\int_{0}^{\infty} s^{m-t}(1+s)^{-m-1} d s>0
$$


para $0<s<m+1$ e $m=0,1,2, \cdots$. Agora a Proposição 2.2.1 e (2.5) implicam

$$
\left\|A^{-t}\right\|_{L(E)} \leq M^{m+1} \frac{\operatorname{sen} \pi t}{\pi} \frac{m !}{(1-t)(2-t) \cdots(m-t)} \int_{0}^{\infty} s^{m-t}(1+s)^{-m-1} d t=M^{m+1}
$$

para $0<t<m+1$ e $m=0,1,2, \cdots$.

Agora suponha que $-1<\operatorname{Re} z<1$. Então pomos

$$
A_{z} e:=\frac{\operatorname{sen} \pi z}{\pi z} \int_{0}^{\infty} s^{z}(s+A)^{-2} A e d s, \quad e \in D(A) .
$$

Observe que

$$
A_{0} e=\int_{0}^{\infty}(s+A)^{-2} d s \quad A e=-\left.(s+A)^{-1} A e\right|_{0} ^{\infty}=e, \quad e \in D(A) .
$$

Adicionalmente, se $\operatorname{Re} z \neq 0$, segue de (2.11) e de (2.17) que

$$
A^{z} e=A^{z-1} A e=\frac{\operatorname{sen} \pi(1-z)}{\pi z} \int_{0}^{\infty} s^{z}(s+A)^{-2} A e d s=A_{z} e
$$

para $e \in D(A)$. Note que

$$
A^{-1} A_{z} \subset B_{z}:=\frac{\operatorname{sen} \pi z}{\pi z} \int_{0}^{\infty} s^{z}(s+A)^{-2} d s \in L(E) .
$$

Seja $\left(e_{j}\right)$ uma seqüência em $D(A)$ tal que $e_{j} \rightarrow 0$ e $A_{z} e_{j} \rightarrow f$ em $E$. Então graças a (2.20), $B_{z} e_{j} \rightarrow 0$ e $B_{z} e_{j} \rightarrow A^{-1} f$, o que implica que $f=0$. Portanto $A_{z}$ é fechável. Motivados por (2.18) e (2.19) fazemos

$$
A^{z}:=\text { fecho de } A_{z}, \quad \operatorname{Re} z=0 .
$$

Daqui por diante, freqüêntemente escreveremos $D\left(A^{z}\right)$ para denotar este espaço vetorial munido com a norma do gráfico de $A^{z}$. Escreveremos $I s(X, Y)$ para denotar o subespaço de $L(X, Y)$ consistindo dos isomorfismos lineares de $X$ sobre $Y$. Com estas considerações já provamos a maior parte do seguinte teorema.

Teorema 2.2.3 Suponha que $A \in \mathcal{P}(E)$. Então a potência fracionária $A^{z} e ́$, para cada $z \in \mathbb{C}$, um operador linear fechado densamente definido em $E$. Se $\operatorname{Re} z<0$ então $A^{z} \in L(E)$ e é dado pela integral

$$
A^{z}=\frac{1}{2 \pi i} \int_{\Gamma}(-\lambda)^{z}(\lambda+A)^{-1} d \lambda
$$

onde $\Gamma$ é qualquer curva simples suave por partes em $\mathbb{C} \backslash \mathbb{R}^{+}$indo de $\infty \mathbf{e}^{-i \varphi}$ a $\infty \mathbf{e}^{i \varphi}$ para algum $\varphi \in(0, \pi)$ tal que $\sigma(-A)$ fica estritamente a esquerda de $\Gamma$. Adicionalmente,

(i) $A^{z}$ é a potência usual de A se z é inteiro.

(ii) $A^{z} e=\frac{\operatorname{sen} \pi z}{\pi z} \int_{0}^{\infty} s^{z}(s+A)^{-2} A e d s, \quad e \in D(A), \quad-1<\operatorname{Re} z<1$. 
(iii) Suponha que ou $m=0,1,2, \cdots, e \in D\left(A^{2 m}\right)$ e $\max \{\operatorname{Re} z, \operatorname{Re} w\}<m$ ou $\operatorname{Re} z, \operatorname{Re} w$ e $\operatorname{Re}(z+$ w) não são nulos e e $\in D\left(A^{u}\right)$ onde $u \in\{z, w, z+w\}$, satisfaz $\operatorname{Re} u=\max \{\operatorname{Re} z, \operatorname{Re} w, \operatorname{Re}(z+$ $w)\}$. Então

$$
A^{z} A^{w} e=A^{z+w} e .
$$

(iv) $A^{z} A^{w}=A^{z+w}, \operatorname{Re} z, \operatorname{Re} w>0$.

(v) $D\left(A^{w}\right) \stackrel{d}{\hookrightarrow} D\left(A^{z}\right) \stackrel{d}{\hookrightarrow} E, 0<\operatorname{Re} z<\operatorname{Re} w$.

(vi) $A^{z} \in I s\left(D\left(A^{z+w}\right), D\left(A^{w}\right)\right) \cap I s\left(D\left(A^{z}\right), E\right), \operatorname{Re} z, \operatorname{Re} w>0$.

(vii) Dado $m=0,1,2, \cdots$, a aplicação

$$
\{z \in \mathbb{C}: \operatorname{Re} z<m\} \rightarrow L\left(D\left(A^{m}\right), E\right), \quad z \mapsto A^{z}
$$

é analítica.

Prova. A primeira parte da afirmativa segue de resultados que precedem o enunciado do teorema.

(i) Segue de (2.1) e de (2.13).

(ii) Se $\operatorname{Re} z \neq 0$, isto foi mostrado em (2.19) e segue da definição de $A^{z}$ se $\operatorname{Re} z=0$.

(iii) Se $\operatorname{Re} z, \operatorname{Re} w$ e $\operatorname{Re}(z+w)$ são todos distintos de zero, isto é uma conseqüência de (2.16) e (2.14). De (ii) e (2.5) concluímos que

$$
\left(z \mapsto A^{z}\right) \in C^{1}\left(\{z \in \mathbb{C}:-1<\operatorname{Re} z<1\}, L(D(A), E) \cap L\left(D\left(A^{2}\right), D(A)\right)\right)
$$

Portanto, suponha que $z, w \in\{\zeta \in \mathbb{C}:-1<\operatorname{Re} \zeta<1\}$. Escolha as seqüências $\left(z_{j}\right),\left(w_{j}\right)$ em

$$
\{z \in \mathbb{C}:-1<\operatorname{Re} z<1\} \backslash\{z \in \mathbb{C}: \operatorname{Re} z=0\}=: Z
$$

tal que $z_{j}+w_{j} \in Z, z_{j} \rightarrow z$ e $w_{j} \rightarrow w$. Então, pelo que já sabemos,

$$
A^{z_{j}} A^{w_{j}} e=A^{z_{j}+w_{j}} e, \quad e \in D\left(A^{2}\right)
$$

Portanto, fazendo $j \rightarrow \infty$, obtemos de (2.22) que (iii) é verdade se $-1<\operatorname{Re} z, \operatorname{Re} w<1$. Suponha que $\operatorname{Re} z=0$ e $\{w \in \mathbb{C}:|\operatorname{Re} w| \geq 1\}$. Fixe $\alpha \in \mathbb{R}$ com $0<\alpha-\operatorname{Re} w<1$. Então

$$
A^{z} A^{w} e=A^{z} A^{w-\alpha} A^{\alpha} e=A^{z+(w-\alpha)} A^{\alpha} e=A^{(z+w-\alpha)+\alpha} e=A^{z+w} e
$$

para $e \in D\left(A^{2 m}\right)$ com $m=2,3, \cdots$ e $\operatorname{Re} w<m$ já que $-1<\operatorname{Re}(w-\alpha)<0$ e $\alpha \neq 0$. 
Finalmente, seja $\operatorname{Re} z \leq-1,1 \leq \operatorname{Re} w$ e $\operatorname{Re}(z+w)=0$. Escrevemos $z=r+s$ com $-1<\operatorname{Re} r<0$. Como as partes reais de $r, w$ e $r+w$ são não nulas e $z, r$ e $s$ tem partes reais negativas, segue que $A^{z}=A^{r} A^{s}$ e $A^{s} A^{w} e=A^{s+w} e$ para $e \in D\left(A^{w}\right)$. Portanto

$$
A^{z} A^{w} e=A^{r} A^{s+w} e, \quad e \in D\left(A^{w}\right) \subset D\left(A^{2 m}\right) .
$$

Logo podemos assumir que $-1<\operatorname{Re} z<0$. Então $\operatorname{Re}(z+w)=0$ implica $0<\operatorname{Re} w<1$, de forma que estamos de volta a situação já considerada. Conseqüêntemente, (iii) foi completamente provado.

(iv) Pelo Teorema 2.2.1 e (iii) é suficiente provar que $e \in D\left(A^{w}\right)$ e $A^{w} e \in D\left(A^{z}\right)$ implica $e \in D\left(A^{w+z}\right)$ se $\operatorname{Re} z>0$ e $\operatorname{Re} w>0$. Seja $f:=A^{z}\left(A^{w} e\right)$. Então segue de (iii) que $e=A^{-w}\left(A^{-z} f\right)=A^{-(w+z)} f \in D\left(A^{w+z}\right)$.

(v) De (2.14) e de (iii) deduzimos que

$$
\left\|A^{z} e\right\|_{E}=\left\|A^{z-w} A^{w} e\right\|_{E} \leq\left\|A^{z-w}\right\|_{L(E)}\left\|A^{w} e\right\|_{E}, \quad e \in D\left(A^{w}\right) .
$$

Como $e \mapsto\left\|A^{u} e\right\|_{E}$ é uma norma equivalente a norma em $D\left(A^{u}\right)$ para Reu $>0$, graças a limitação de $A^{-u}$, segue que $D\left(A^{w}\right) \hookrightarrow D\left(A^{z}\right) \hookrightarrow E$.

Dado $e \in D\left(A^{z}\right)$ faça $f:=A^{z} e \in E$. Como $D\left(A^{w-z}\right)$ é denso em $E$, dado $\epsilon>0$ podemos encontrar $u \in D\left(A^{w-z}\right)$ tal que $\|u-f\|_{E}<\epsilon$. Portanto

$$
v:=A^{-z} u \in D\left(A^{w}\right) \quad \text { e } \quad\left\|A^{z}(v-e)\right\|_{E}=\|u-f\|_{E}<\epsilon .
$$

Isto mostra que $D\left(A^{w}\right)$ é denso em $D\left(A^{z}\right)$ que, junto com (2.15) implica a afirmativa.

(vi) A primeira afirmativa segue de (iv) e a segunda é trivial.

(vii) Graças ao Teorema 2.2.1 e (2.22), podemos assumir que $m \geq 2$. Desde que (v) implica

$$
L(D(A), E) \hookrightarrow L\left(D\left(A^{m}\right), E\right),
$$

concluímos que

$$
\left(z \mapsto A^{z}\right) \in C^{1}\left(\{z \in \mathbb{C} ; \operatorname{Re} z<1\}, L\left(D\left(A^{m}\right), E\right)\right) .
$$

Se $0<\operatorname{Re} z<m$ então (iii) implica que $A^{z} e=A^{z-m} A^{m} e$ para $e \in D\left(A^{m}\right)$. Portanto o Teorema 2.2.1 garante que

$$
\left(z \mapsto A^{z}\right) \in C^{1}\left(\{z \in \mathbb{C} ; 0<\operatorname{Re} z<m\}, L\left(D\left(A^{m}\right), E\right)\right)
$$

Isto juntamente com (2.24) prova o teorema. 
Note que se $-A$ é o gerador infinitesimal de um semigrupo fortemente contínuo com decaimento exponencial em $E$ então $A$ é do tipo positivo. Neste caso podemos obter outra fórmula de representação útil para $A^{z} \operatorname{com} \operatorname{Re} z>0$.

Teorema 2.2.4 Suponha que A é o gerador de um semigrupo fortemente contínuo com decaimento exponencial. Então

$$
A^{-z}=\frac{1}{\Gamma(z)} \int_{0}^{\infty} t^{z-1} \mathbf{e}^{-t A} d t, \quad \operatorname{Re} z>0
$$

Prova. É uma conseqüência fácil de

$$
\left\|\int_{0}^{\infty} t^{z-1} \mathbf{e}^{-t A} d t\right\|_{L(E)} \leq M \int_{0}^{\infty} t^{\operatorname{Re} z-1} \mathbf{e}^{-\sigma t} d t
$$

e das propriedades conhecidas da função $\Gamma$ que a aplicação

$$
\{z \in \mathbb{C}: \operatorname{Re} z>0\} \rightarrow L(E), \quad z \mapsto \frac{1}{\Gamma(z)} \int_{0}^{\infty} t^{z-1} \mathbf{e}^{-t A} d t
$$

é analítica. Portanto, graças ao Teorema 2.2.1 e ao Teorema da identidade de funções análiticas é suficiente provar a igualdade para $0<z<1$.

Dado $z \in(0,1)$,

$$
A^{-z}=\frac{\operatorname{sen} \pi z}{\pi} \int_{0}^{\infty} s^{-z}(s+A)^{-1} d s
$$

pela Proposição 2.2.1. Por outro lado sabemos da teoria de semigrupos que

$$
(s+A)^{-1}=\int_{0}^{\infty} \mathbf{e}^{-s t} \mathbf{e}^{-t A} d t, \quad s>0 .
$$

Portanto pelo Teorema de Fubini

$$
\begin{aligned}
A^{-z} & =\frac{\operatorname{sen} \pi z}{\pi} \int_{0}^{\infty} s^{-z} \int_{0}^{\infty} \mathbf{e}^{-s t} \mathbf{e}^{-t A} d t d s=\frac{\operatorname{sen} \pi z}{\pi} \int_{0}^{\infty} \mathbf{e}^{-t A} \int_{0}^{\infty} s^{-z} \mathbf{e}^{-t s} d s d t \\
& =\frac{\operatorname{sen} \pi z}{\pi} \Gamma(1-z) \int_{0}^{\infty} t^{z-1} \mathbf{e}^{-t A} d t .
\end{aligned}
$$

Assim a afirmativa segue da fórmula

$$
\Gamma(z) \Gamma(1-z)=\pi / \operatorname{sen} \pi z
$$

que é conseqüência da relação anterior para $A=I$. 


\subsection{Potências de Potências Fracionárias}

Nesta seção apresentamos dois resultados. O primeiro deles, devido a T. Kato (veja [15]), estabelece uma fórmula para o operador resolvente de potências fracionárias. Esta fórmula é utilizada para mostrar (neste mesmo teorema) que é possível calcular potências fracionárias de potências fracionárias. Este mesmo resultado ainda estabelece, no caso em que $A$ gera um semigrupo fortemente contínuo com decaimento exponencial, uma fórmula (devida a Yosida [22]) para um semigrupo analítico gerado por $-A^{\alpha}$ em função do semigrupo gerado por $-A$. O segundo resultado é uma conseqüência simples do primeiro e estabelece o seguinte teorema de reiteração: $\left(A^{\alpha}\right)^{\beta}=A^{\alpha \beta}$.

Definição 2.3.1 Dizemos que A é do tipo $(\omega, M)$ em um espaço de Banach $E$ se $A$ é fechado, densamente definido e o resolvente de $-A$ contém um setor aberto $\{\lambda \in \mathbb{C}:|\arg \lambda|<\pi-\omega\}$ e $\lambda(\lambda+A)^{-1}$ é uniformemente limitado em cada setor menor $\{\lambda \in \mathbb{C}:|\arg \lambda|<\pi-\omega-\epsilon\}, \epsilon>0$ $e\left\|\lambda(\lambda+A)^{-1}\right\| \leq M, \lambda \geq 0$ (ver [15]).

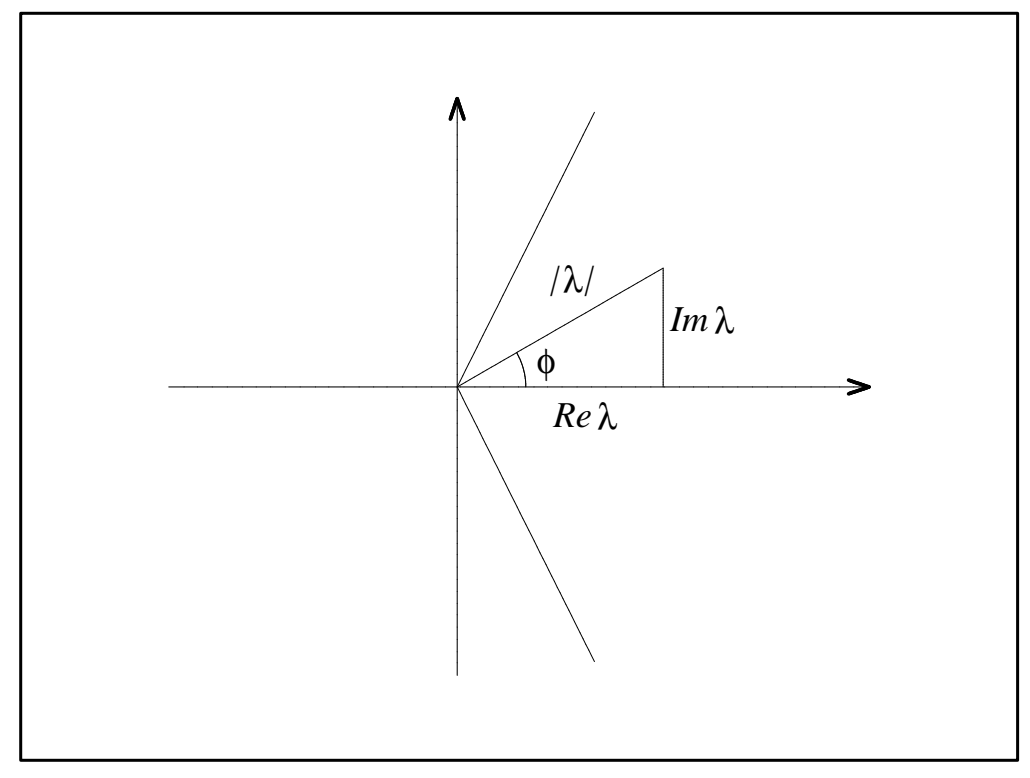

Figura 2.2:

É claro que se $A$ é gerador de um semigrupo fortemente contínuo $\{T(t): t \geq 0\}$ tal que $\|T(t)\| \leq M$ para todo $t \geq 0$ então $A$ é do tipo $(\pi / 2, M)$ (basta observar que em qualquer setor $\Sigma_{\phi} \operatorname{com} \phi<\pi / 2$ temos $\operatorname{Re} \lambda \geq|\lambda| \cos \phi$ e que neste setor $\left.\left\|(\lambda+A)^{-1}\right\| \leq M / \operatorname{Re} \lambda\right)$. Já vimos também que se $A$ é do tipo $(\omega, M) \operatorname{com} \omega<\pi / 2$ então $-A$ é gerador de um semigrupo analítico 
$\left\{T(t): t \in \Sigma_{\phi-\pi / 2}\right\}$ e neste caso

$$
T(t)=\frac{1}{2 \pi i} \int_{\Gamma^{\prime}} \mathbf{e}^{\lambda t}(\lambda+A)^{-1} d \lambda,
$$

onde a trajetória de integração $\Gamma^{\prime}$ percorre o setor $\{\lambda \in \mathbb{C}:|\arg \lambda|<\pi-\omega\}$ de $\infty \mathbf{e}^{-i \nu}$ a $\infty \mathbf{e}^{i \nu}$, $\pi / 2<\nu<\pi-\omega$.

O Teorema a seguir terá importância fundamental na demonstração de que para todo operador dissipativo $A$ em um espaço de Hilbert $H$ com $0 \in \rho(A)$ podemos definir o grupo fortemente contínuo $\left\{A^{i t} \in L(H): t \in \mathbb{R}\right\}$. Isto, por sua vez, tem importância fundamental na caracterização dos espaços de potência fracionária $D\left(A^{\alpha}\right)$ que por sua vez é ferramenta indispensável para lidar com expoentes críticos em problemas semilineares parabólicos.

Teorema 2.3.1 (Kato) Seja A um operador do tipo $(\omega, M)$ em um espaço de Banach $E$ com $0 \in \rho(A)$ e $0<\alpha<1$, então

$$
\left(\lambda+A^{\alpha}\right)^{-1}=\frac{1}{2 \pi i} \int_{\Gamma} \frac{1}{\lambda+(-\mu)^{\alpha}}(\mu+A)^{-1} d \mu, \quad \lambda \geq 0,
$$

onde $\Gamma$ é um contorno como em (2.21). Deformando $\Gamma$ sobre $\mathbb{R}^{+}$segue que

$$
\left(\lambda+A^{\alpha}\right)^{-1}=\frac{\operatorname{sen} \pi \alpha}{\pi} \int_{0}^{\infty} \frac{s^{\alpha}(s+A)^{-1}}{s^{2 \alpha}+2 \lambda s^{\alpha} \cos \pi \alpha+\lambda^{2}} d s, \quad \lambda \geq 0 .
$$

Além disso, $A^{\alpha}$ é do tipo $(\alpha \omega, M)$. Se $\alpha \omega<\pi / 2$ então $-A^{\alpha}$ é o gerador infinitesimal de um semigrupo analítico $\left\{T_{\alpha}(t): t \in \Sigma_{\pi / 2-\alpha \omega}\right\}$. No caso em que $-A$ gera um semigrupo fortemente contínuo com decaimento exponencial, $T_{\alpha}(t)$ é dado por

$$
T_{\alpha}(t)=\frac{1}{2 \pi i} \int_{0}^{\infty} T(\tau) \int_{\Gamma} \mathbf{e}^{-\tau \mu+t(-\mu)^{\alpha}} d \mu d \tau .
$$

Prova. Mostremos que a integral em (2.27) é absolutamente convergente. Como $A$ é do tipo $(\omega, M)$ então $A$ é densamente definido e fechado. Além disso,

$$
\left\|s(s+A)^{-1}\right\| \leq M, \quad s \geq 0
$$

Lembre-se que a aplicação que a cada $\lambda \in \rho(A)$ associa $(\lambda+A)^{-1}$ é contínua.

Se $\lambda=0$, então

$$
\begin{aligned}
\left\|\frac{\operatorname{sen} \pi \alpha}{\pi} \int_{0}^{\infty} \frac{s^{\alpha}(s+A)^{-1}}{s^{2 \alpha}} d s\right\| & \leq\left\|\frac{\operatorname{sen} \pi \alpha}{\pi} \int_{0}^{1} \frac{s^{\alpha}(s+A)^{-1}}{s^{2 \alpha}} d s\right\| \\
& +\left\|\frac{\operatorname{sen} \pi \alpha}{\pi} \int_{1}^{\infty} \frac{s^{\alpha}(s+A)^{-1}}{s^{2 \alpha}} d s\right\| \\
& \leq \frac{1}{\pi} N \int_{0}^{1} s^{-\alpha} d s+\frac{1}{\pi} M \int_{1}^{\infty} s^{-(\alpha+1)} d s \\
& =\frac{1}{\pi}\left(\frac{N}{1-\alpha}+\frac{M}{\alpha}\right)
\end{aligned}
$$


Se $\lambda \neq 0$, então o termo que controla a convergência é $s^{2 \alpha}$. E um argumento similar ao anterior, mostra que $\frac{\operatorname{sen} \pi \alpha}{\pi} \int_{0}^{\infty} \frac{s^{\alpha}(s+A)^{-1}}{s^{2 \alpha}+2 \lambda s^{\alpha} \cos \pi \alpha+\lambda^{\alpha}} d s$ é absolutamente convergente. Denote por $R(\lambda)$ o operador linear limitado definido pelo lado direito de (2.26). Agora deformando $\Gamma$ sobre $\mathbb{R}^{+}$, vamos mostrar que $R(\lambda)$ é dado por (2.27). Observe que (2.26) pode ser reescrito na forma

$$
\left(\lambda+A^{\alpha}\right)^{-1}=-\frac{1}{2 \pi i} \int_{\tilde{\Gamma}} \frac{1}{\lambda+\mu^{\alpha}}(\mu-A)^{-1} d \mu, \quad \lambda \geq 0
$$

onde escolhemos $\tilde{\Gamma} \subset \mathbb{C} / \mathbb{R}^{-}$, consistindo de:

$$
\left\{-s \mathbf{e}^{-i(\pi-\epsilon)}: s \in(-\infty,-r]\right\}, \quad\left\{r \mathbf{e}^{i \varphi}:|\varphi| \leq \pi-\epsilon\right\}, \quad\left\{s \mathbf{e}^{i(\pi-\epsilon)}: s \in[r, \infty)\right\}
$$

onde $\epsilon>0$ e $r>0$ são suficientemente pequenos.

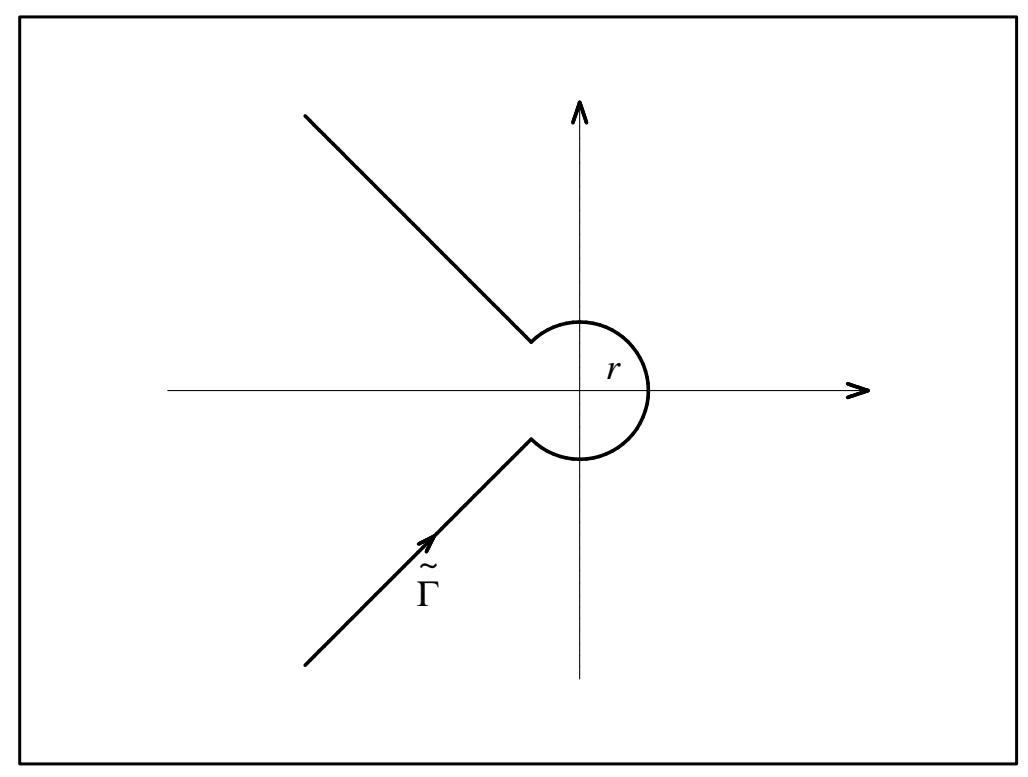

Figura 2.3:

Segue que

$$
\begin{aligned}
\left(\lambda+A^{\alpha}\right)^{-1} & =-\frac{1}{2 \pi i} \int_{-\infty}^{-r} \frac{1}{\lambda+\left(-s \mathbf{e}^{-i(\pi-\epsilon)}\right)^{\alpha}}\left(-s \mathbf{e}^{-i(\pi-\epsilon)}-A\right)^{-1}\left(-\mathbf{e}^{-i(\pi-\epsilon)}\right) d s \\
& -\frac{1}{2 \pi i} \int_{-(\pi-\epsilon)}^{\pi-\epsilon} \frac{1}{\lambda+\left(r \mathbf{e}^{i \varphi}\right)^{\alpha}}\left(r \mathbf{e}^{i \varphi}-A\right)^{-1} r i \mathbf{e}^{i \varphi} d \varphi \\
& -\frac{1}{2 \pi i} \int_{r}^{\infty} \frac{1}{\lambda+\left(s \mathbf{e}^{i(\pi-\epsilon)}\right)^{\alpha}}\left(s \mathbf{e}^{i(\pi-\epsilon)}-A\right)^{-1} \mathbf{e}^{i(\pi-\epsilon)} d s .
\end{aligned}
$$

Dado $r>0$, temos

$$
\frac{1}{\lambda+\mathbf{e}^{\alpha(\ln (-s)-i(\pi-\epsilon))}}\left(-s \mathbf{e}^{-i(\pi-\epsilon)}-A\right)^{-1}\left(-\mathbf{e}^{-i(\pi-\epsilon)}\right) \stackrel{\epsilon \rightarrow 0}{\longrightarrow} \frac{1}{\lambda+(-s)^{\alpha} \mathbf{e}^{-i \pi \alpha}}(s-A)^{-1}
$$


$\mathrm{e}$

$$
\begin{aligned}
& \left\|\frac{1}{\lambda+\mathbf{e}^{\alpha(\ln |s|-i(\pi-\epsilon))}}\left(-s \mathbf{e}^{-i(\pi-\epsilon)}-A\right)^{-1}\left(-\mathbf{e}^{-i(\pi-\epsilon)}\right)\right\| \leq \frac{2 M+1}{1-s} \frac{1}{\left|\lambda+\mathbf{e}^{\alpha(\ln (-s)-i(\pi-\epsilon))}\right|} \\
& \leq \frac{2 M+1}{1-s} \frac{1}{(-s)^{\alpha}-\lambda} \\
& \leq(2 M+1)(-s)^{-\alpha-1},
\end{aligned}
$$

onde usamos que

$$
\left|\lambda+\mathbf{e}^{\alpha(\ln (-s)-i(\pi-\epsilon))}\right| \geq\left|\mathbf{e}^{\alpha(\ln (-s)-i(\pi-\epsilon))}\right|-|\lambda|=(-s)^{\alpha}-\lambda
$$

e ainda, na estimativa anterior a função dominante é integrável sobre $(-\infty,-r)$, pois $0<\alpha<1$. Segue do Teorema da Convergência Dominada, que

$$
\begin{aligned}
& \int_{-\infty}^{-r} \frac{1}{\lambda+\mathbf{e}^{\alpha(\ln |s|-i(\pi-\epsilon))}}\left(-s \mathbf{e}^{-i(\pi-\epsilon)}-A\right)^{-1}\left(-\mathbf{e}^{-i(\pi-\epsilon)}\right) d s \\
& \stackrel{\epsilon \rightarrow 0}{\longrightarrow} \int_{-\infty}^{-r} \frac{1}{\lambda+(-s)^{\alpha} \mathbf{e}^{-i \pi \alpha}}(s-A)^{-1} d s .
\end{aligned}
$$

De maneira análoga, para $r>0$, temos

$$
\frac{1}{\lambda+\mathbf{e}^{\alpha(\ln s+i(\pi-\epsilon))}}\left(s \mathbf{e}^{i(\pi-\epsilon)}-A\right)^{-1} \mathbf{e}^{i(\pi-\epsilon)} \stackrel{\epsilon \rightarrow 0}{\longrightarrow}-\frac{1}{\lambda+s^{\alpha} \mathbf{e}^{i \pi \alpha}}(-s-A)^{-1}
$$

e

$$
\begin{aligned}
\left\|\frac{1}{\lambda+\left(s \mathbf{e}^{i(\pi-\epsilon)}\right)^{\alpha}}\left(s \mathbf{e}^{i(\pi-\epsilon)}-A\right)^{-1} \mathbf{e}^{i(\pi-\epsilon)}\right\| & \leq \frac{2 M+1}{1+s} \frac{1}{\left|\lambda+s^{\alpha} \mathbf{e}^{i \alpha(\pi-\epsilon)}\right|} \\
& \leq(2 M+1) s^{-\alpha-1}
\end{aligned}
$$

onde a função dominante é integrável sobre $(r, \infty)$, pois $0<\alpha<1$. Então, do Teorema da Convergência Dominada, segue que

$$
\int_{r}^{\infty} \frac{1}{\lambda+\left(s \mathbf{e}^{i(\pi-\epsilon)}\right)^{\alpha}}\left(s \mathbf{e}^{i(\pi-\epsilon)}-A\right)^{-1} \mathbf{e}^{i(\pi-\epsilon)} d s \stackrel{\epsilon \rightarrow 0}{\longrightarrow}-\int_{r}^{\infty} \frac{1}{\lambda+s^{\alpha} \mathbf{e}^{i \pi \alpha}}(-s-A)^{-1} d s
$$

Ainda, como

$$
\int_{-\pi}^{\pi}\left\|\frac{1}{\lambda+\mathbf{e}^{\alpha(\ln r+i \varphi)}}\left(r \mathbf{e}^{i \varphi}-A\right)^{-1} r i \mathbf{e}^{i \varphi}\right\| d \varphi \leq \frac{(2 M+1)}{1+r} \int_{-\pi}^{\pi} \frac{1}{r^{\alpha}-\lambda} d \varphi<\infty,
$$

obtemos que

$$
\int_{-(\pi-\epsilon)}^{\pi-\epsilon} \frac{1}{\lambda+\mathbf{e}^{\alpha(\ln r+i \varphi)}}\left(r \mathbf{e}^{i \varphi}-A\right)^{-1} r i \mathbf{e}^{i \varphi} \stackrel{\epsilon \rightarrow 0}{\longrightarrow} \int_{-\pi}^{\pi} \frac{1}{\lambda+\mathbf{e}^{\alpha(\ln r+i \varphi)}}\left(r \mathbf{e}^{i \varphi}-A\right)^{-1} r i \mathbf{e}^{i \varphi} d \varphi
$$

Então de (2.29), (2.30) e (2.31) segue que

$$
\begin{aligned}
\left(\lambda+A^{\alpha}\right)^{-1} & =\frac{1}{2 \pi i} \int_{-\infty}^{-r} \frac{1}{\lambda+(-s)^{\alpha} \mathbf{e}^{-i \pi \alpha}}(-s+A)^{-1} d s \\
& -\frac{1}{2 \pi i} \int_{-\pi}^{\pi} \frac{1}{\lambda+\mathbf{e}^{\alpha(\ln r+i \varphi)}}\left(r \mathbf{e}^{i \varphi}-A\right)^{-1} r i \mathbf{e}^{i \varphi} d \varphi \\
& -\frac{1}{2 \pi i} \int_{r}^{\infty} \frac{1}{\lambda+s^{\alpha} \mathbf{e}^{i \pi \alpha}}(s+A)^{-1} d s .
\end{aligned}
$$


Ainda, se $\lambda=0$, então

$$
\left\|\frac{1}{\mathbf{e}^{\alpha(\ln r+i \varphi)}}\left(r \mathbf{e}^{i \varphi}-A\right)^{-1} r i \mathbf{e}^{i \varphi}\right\| \leq(2 M+1) \frac{r}{r^{\alpha}}=(2 M+1) r^{1-\alpha} \stackrel{r \rightarrow 0}{\longrightarrow} 0,
$$

pois, $0<\alpha<1$. Se $\lambda \neq 0$, então

$$
\left\|\frac{1}{\lambda+\mathbf{e}^{\alpha(\ln r+i \varphi)}}\left(r \mathbf{e}^{i \varphi}-A\right)^{-1} r i \mathbf{e}^{i \varphi}\right\| \leq \frac{(2 M+1) r}{\lambda-r^{\alpha}} \stackrel{r \rightarrow 0}{\longrightarrow} 0
$$

Do Teorema da Convergência Dominada, segue que

$$
\begin{aligned}
\left(\lambda+A^{\alpha}\right)^{-1} & =\frac{1}{2 \pi i} \int_{0}^{\infty} \frac{1}{\lambda+s^{\alpha} \mathbf{e}^{-i \pi \alpha}}(s+A)^{-1} d s \\
& -\frac{1}{2 \pi i} \int_{0}^{\infty} \frac{1}{\lambda+s^{\alpha} \mathbf{e}^{i \pi \alpha}}(s+A)^{-1} d s \\
& =\frac{1}{2 \pi i} \int_{0}^{\infty}\left(\frac{1}{\lambda+s^{\alpha} \mathbf{e}^{-i \pi \alpha}}-\frac{1}{\lambda+s^{\alpha} \mathbf{e}^{i \pi \alpha}}\right)(s+A)^{-1} d s \\
& =\frac{1}{2 \pi i} \int_{0}^{\infty}\left(\frac{\mathbf{e}^{i \pi \alpha}-\mathbf{e}^{-i \pi \alpha}}{\left(\lambda+s^{\alpha} \mathbf{e}^{-i \pi \alpha}\right)\left(\lambda+s^{\alpha} \mathbf{e}^{i \pi \alpha}\right)}\right) s^{\alpha}(s+A)^{-1} d s \\
& =\frac{\operatorname{sen} \pi \alpha}{\pi} \int_{0}^{\infty} \frac{s^{\alpha}(s+A)^{-1}}{\lambda^{2}+2 \lambda s^{\alpha} \cos \pi \alpha+s^{2 \alpha}} d s .
\end{aligned}
$$

De (2.26) obtemos

$$
\begin{aligned}
\left(\lambda^{\prime}-\lambda\right) R(\lambda) R\left(\lambda^{\prime}\right) & =\frac{1}{(2 \pi i)^{2}} \int_{\Gamma} \int_{\Gamma^{\prime}} \frac{\lambda^{\prime}-\lambda}{\left(\lambda+(-\mu)^{\alpha}\right)\left(\lambda^{\prime}+(-\nu)^{\alpha}\right)}(\mu+A)^{-1}(\nu+A)^{-1} d \nu d \mu \\
& =\frac{1}{(2 \pi i)^{2}} \int_{\Gamma} \int_{\Gamma^{\prime}} \frac{\lambda^{\prime}-\lambda}{\left(\lambda+(-\mu)^{\alpha}\right)\left(\lambda^{\prime}+(-\nu)^{\alpha}\right)} \frac{(\mu+A)^{-1}-(\nu+A)^{-1}}{\nu-\mu} d \nu d \mu \\
& =\frac{1}{2 \pi i} \int_{\Gamma} \frac{\lambda^{\prime}-\lambda}{\lambda+(-\mu)^{\alpha}}\left(\frac{1}{2 \pi i} \int_{\Gamma^{\prime}} \frac{1}{(\nu-\mu)} \frac{1}{\lambda^{\prime}+(-\nu)^{\alpha}} d \nu\right)(\mu+A)^{-1} d \mu \\
& +\frac{1}{2 \pi i} \int_{\Gamma^{\prime}} \frac{\lambda^{\prime}-\lambda}{\lambda+(-\nu)^{\alpha}}\left(\frac{1}{2 \pi i} \int_{\Gamma} \frac{1}{(\mu-\nu)} \frac{1}{\lambda+(-\nu)^{\alpha}} d \mu\right)(\nu+A)^{-1} d \nu \\
& =\frac{1}{2 \pi i} \int_{\Gamma^{\prime}} \frac{\lambda^{\prime}-\lambda}{\left(\lambda+(-\nu)^{\alpha}\right)\left(\lambda^{\prime}+(-\nu)^{\alpha}\right)}(\nu+A)^{-1} d \nu \\
& =\frac{1}{2 \pi i} \int_{\Gamma^{\prime}} \frac{1}{\lambda+(-\nu)^{\alpha}}(\nu+A)^{-1} d \nu-\frac{1}{2 \pi i} \int_{\Gamma^{\prime}} \frac{1}{\lambda^{\prime}+(-\nu)^{\alpha}}(\nu+A)^{-1} d \nu \\
& =R(\lambda)-R\left(\lambda^{\prime}\right)
\end{aligned}
$$

onde usamos o Teorema de Cauchy, para concluir que

$$
\frac{1}{2 \pi i} \int_{\Gamma^{\prime}} \frac{1}{\lambda^{\prime}+(-\nu)^{\alpha}} \frac{1}{\nu-\mu} d \nu=0
$$

e $\Gamma^{\prime}$ é um contorno com as mesmas propriedades de $\Gamma$ à direita de $\Gamma$. Vamos mostrar que

$$
\int_{\gamma_{R}} \frac{1}{\lambda^{\prime}+(-\nu)^{\alpha}} \frac{1}{\nu-\mu} d \nu \rightarrow 0, \quad R \rightarrow \infty
$$




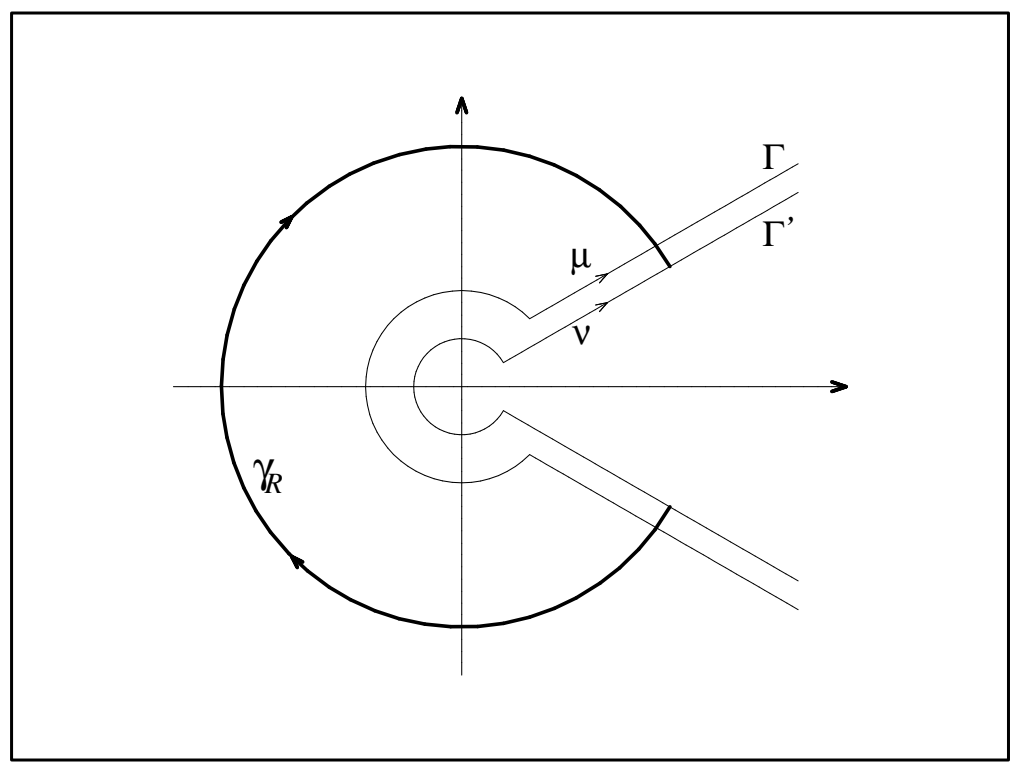

Figura 2.4:

onde $\gamma_{R}$ é dado na Figura 2.4, então

$$
\begin{aligned}
\left\|\int_{\gamma_{R}} \frac{1}{\lambda^{\prime}+(-\nu)^{\alpha}} \frac{1}{\nu-\mu} d \nu\right\| & =\left\|\int_{2 \pi-\theta}^{\theta} \frac{1}{\lambda^{\prime}+\left(-R \mathbf{e}^{i \tau}\right)^{\alpha}} \frac{R i \mathbf{e}^{i \tau}}{R \mathbf{e}^{i \tau}-\mu} d \tau\right\| \\
& \leq \int_{2 \pi-\theta}^{\theta} \frac{1}{\mid \lambda^{\prime}+\mathbf{e}^{\alpha(\ln R+i(-\pi+\tau)) \mid}} \frac{R}{\left|R \mathbf{e}^{i \tau}+\mu\right|} d \tau \\
& \leq \int_{2 \pi-\theta}^{\theta} \frac{R}{\left(R^{\alpha}-\lambda^{\prime}\right)(R-|\mu|)} d \tau \\
& =\frac{2 R(-\pi+\theta)}{\left(R^{\alpha}-\lambda^{\prime}\right)(R-|\mu|)} \\
& =\frac{2(-\pi+\theta)}{\left(R^{\alpha}-\lambda^{\prime}\right)\left(1-\frac{|\mu|}{R}\right)} \stackrel{R \rightarrow \infty}{\longrightarrow} 0 .
\end{aligned}
$$

onde $\theta$ é como na Figura 2.1. Como $R(0)=A^{-\alpha}$ tem imagem densa e núcleo trivial segue do Teorema 1.4.1 que $\left(\lambda+A^{\alpha}\right)^{-1}=R(\lambda)$.

Agora note que $R(\lambda)$ pode ser continuado analiticamente para o setor $\{\lambda \in \mathbb{C}:|\arg \lambda|<$ $\pi-\alpha \omega\}$. Para ver isto é suficiente considerar a integral em (2.26) nos raios $\arg \mu= \pm(\pi-\omega-\epsilon)$, $\epsilon>0$ pequeno, $|\mu| \geq 1$ e observar que sobre estes raios

$$
\begin{aligned}
\left|\lambda+(-\mu)^{\alpha}\right| & =\left.|| \lambda\left|\mathbf{e}^{i \arg \lambda}+\right| \mu\right|^{\alpha} \mathbf{e}^{\mp i \alpha(\omega+\epsilon)} \mid \\
& =\left.|| \mu\right|^{\alpha} \mathbf{e}^{\mp i \alpha(\omega+\epsilon)}\left(1+\frac{|\lambda|}{|\mu|^{\alpha}} \mathbf{e}^{i(\arg \lambda \pm \alpha(\omega+\epsilon))}\right) \mid \\
& =|\mu|^{\alpha}\left|\frac{|\lambda|}{|\mu|^{\alpha}} \mathbf{e}^{i(\arg \lambda \pm \alpha(\omega+\epsilon))}+1\right|
\end{aligned}
$$


de onde obtemos que $\left|\lambda+(-\mu)^{\alpha}\right|$ é uma função contínua de $\mu$ para $\mu \in \Gamma$ tal que

$$
\inf _{|\mu| \geq 1}\left|\frac{|\lambda|}{|\mu|^{\alpha}} \mathbf{e}^{i(\arg \lambda \pm \alpha(\omega+\epsilon))}+1\right|=\delta>0
$$

e portanto

$$
\left|\lambda+(-\mu)^{\alpha}\right|^{-1} \leq \delta^{-1} \frac{1}{|\mu|^{\alpha}}
$$

sempre que $|\arg | \lambda<\pi-\alpha(\omega+\epsilon)$. Estes cálculos também mostram que $\lambda\left(\lambda+A^{\alpha}\right)^{-1}$ é limitada uniformemente em qualquer setor fechado contido em $\Sigma_{\pi-\alpha \omega}$. Em particular, para $\lambda>0,(2.27)$ nos dá

$$
\left\|\left(\lambda+A^{\alpha}\right)^{-1}\right\| \leq \frac{\operatorname{sen} \pi \alpha}{\pi} \int_{0}^{\infty} \frac{\mu^{\alpha}}{\lambda^{2}+2 \lambda \mu^{\alpha} \cos \pi \alpha+\mu^{2 \alpha}} \frac{M}{\mu} d \mu=\frac{M}{\lambda} .
$$

Isto completa a prova de que $A^{\alpha}$ é do tipo $(\alpha \omega, M)$.

Agora está claro que se $\alpha \omega<\pi / 2$ então $\left\{T_{\alpha}(t): t \in \Sigma_{\pi-\alpha \omega}\right\}$ é um semigrupo analítico. Resta apenas mostrar que este semigrupo é dado por (2.28) no caso em que $-A$ gera um semigrupo fortemente contínuo com decaimento exponencial. Neste caso existe $\epsilon>0$ tal que $-(A-\epsilon)$ é o gerador de um semigrupo fortemente contínuo e limitado de operadores de forma que $\{\lambda \in$ $\mathbb{C}: \operatorname{Re} \lambda>-\epsilon\} \subset \rho(-A)$. Como $\omega=\pi / 2$, a trajetória $\Gamma$ em (2.26) pode ser escolhida de forma que $\operatorname{Re} \mu>-\epsilon$ e $\left|\arg (-\mu)^{\alpha}\right| \leq \phi<\pi / 2$ para $\mu \in \Gamma$. Então (2.26) é válida para todo $\lambda$ com $|\arg \lambda| \leq \pi-\phi(>\pi / 2)$. Escolha a trajetória $\Gamma^{\prime}$ em (2.25) tal que esta condição está satisfeita para todo $\lambda$ em $\Gamma^{\prime}$. Então, lembrando que $R(\lambda)=\left(\lambda+A^{\alpha}\right)^{-1}$, temos que

$$
\begin{aligned}
T_{\alpha}(t) & =\frac{1}{2 \pi i} \int_{\Gamma^{\prime}} \mathbf{e}^{\lambda t}\left(\lambda+A^{\alpha}\right)^{-1} d \lambda \\
& =\frac{1}{(2 \pi i)^{2}} \int_{\Gamma^{\prime}} \mathbf{e}^{\lambda t} \int_{\Gamma}\left(\lambda+(-\mu)^{\alpha}\right)^{-1}(\mu+A)^{-1} d \mu d \lambda \\
& =\frac{1}{(2 \pi i)^{2}} \int_{\Gamma} \int_{\Gamma^{\prime}} \frac{\mathbf{e}^{\lambda}(\mu+A)^{-1}}{\lambda+(-\mu)^{\alpha}} d \lambda d \mu \\
& =\frac{1}{2 \pi i} \int_{\Gamma}(\mu+A)^{-1}\left(\frac{1}{2 \pi i} \int_{\Gamma^{\prime}} \frac{\mathbf{e}^{\lambda t}}{\lambda+(-\mu)^{\alpha}} d \lambda\right) d \mu \\
& =\frac{1}{2 \pi i} \int_{\Gamma} \mathbf{e}^{t(-\mu)^{\alpha}}(\mu+A)^{-1} d \mu \\
& =\frac{1}{2 \pi i} \int_{\Gamma} \mathbf{e}^{t(-\mu)^{\alpha}} \int_{0}^{\infty} \mathbf{e}^{-\tau \mu} T(\tau) d \tau d \mu \\
& =\frac{1}{2 \pi i} \int_{0}^{\infty} T(\tau) \int_{\Gamma} \mathbf{e}^{-\tau \mu+t(-\mu)^{\alpha}} d \mu d \tau .
\end{aligned}
$$

Mostrando que $T_{\alpha}(t)$ é dado por (2.28).

Observação 2.3.1 O teorema anterior continua válido se eliminarmos a hipótese $0 \in \rho(A)$, (veja [15]). 
Segue do Teorema 2.3.1 e do Teorema 2.2.3 que as potências fracionárias $\left(A^{\alpha}\right)^{z}$ estão bem definidas para $z \in \mathbb{C}$ e $\alpha \in(0,1)$. No teorema a seguir nos restringimos, por simplicidade, ao caso $z \in \mathbb{R}$.

Teorema 2.3.2 Suponha que $A \in \mathcal{P}(E)$ e que $0<\alpha<1$. Então $\left(A^{\alpha}\right)^{\beta}=A^{\alpha \beta}$ para $\beta \in \mathbb{R}$.

Prova. Como $A \in \mathcal{P}(E)$, então existe $M \geq 1$ tal que

$$
(1+s)\left\|(s+A)^{-1}\right\| \leq M, \quad s \in \mathbb{R}^{+}
$$

e ainda, $A$ é fechado, densamente definido e $\mathbb{R}^{+} \subset \rho(-A)$. Pelo Teorema 2.3.1, obtemos que $A^{\alpha}$ é fechado, densamente definido e

$$
\begin{aligned}
\left\|\left(s+A^{\alpha}\right)^{-1}\right\| & \leq \frac{1}{2 \pi i} \int_{\Gamma} \frac{1}{s+(-\mu)^{\alpha}}\left\|(\mu+A)^{-1}\right\| d \mu \\
& \leq \frac{1}{2 \pi i} \int_{\Gamma} \frac{1}{s+(-\mu)^{\alpha}} \frac{M}{1+\mu} d \mu \\
& =\frac{M}{1+s}
\end{aligned}
$$

Logo, $A^{\alpha} \in \mathcal{P}(E)$ com constante $M$. Do Teorema 2.3.1

$$
\left(\mu+A^{\alpha}\right)^{-1}=\frac{1}{2 \pi i} \int_{\Gamma} \frac{(\lambda+A)^{-1}}{\mu+(-\lambda)^{\alpha}} d \lambda, \quad \mu \in \Sigma_{M}
$$

onde $\Gamma$ é uma curva suave por partes indo de $\infty \mathbf{e}^{-i \nu}$ até $\infty \mathbf{e}^{i \nu}$ em $\Sigma_{M} \backslash \mathbb{R}^{+}$, para $\nu$ suficientemente pequenos. Portanto, por (2.21) e pela fórmula integral de Cauchy,

$$
\begin{aligned}
\left(A^{\alpha}\right)^{-\beta} & =\frac{1}{2 \pi i} \int_{\Gamma^{\prime}}(-\mu)^{-\beta}\left(\mu+A^{\alpha}\right)^{-1} d \mu \\
& =\frac{1}{2 \pi i} \int_{\Gamma^{\prime}}(-\mu)^{-\beta}\left(\frac{1}{2 \pi i} \int_{\Gamma} \frac{(\lambda+A)^{-1}}{\mu+(-\lambda)^{\alpha}} d \lambda\right) d \mu \\
& =\frac{1}{2 \pi i} \int_{\Gamma}(\lambda+A)^{-1}\left(\frac{1}{2 \pi i} \int_{\Gamma^{\prime}} \frac{(-\mu)^{-\beta}}{\mu+(-\lambda)^{\alpha}} d \mu\right) d \lambda \\
& =\frac{1}{2 \pi i} \int_{\Gamma}(-\lambda)^{-\alpha \beta}(\lambda+A)^{-1} d \lambda=A^{-\alpha \beta}
\end{aligned}
$$

para $\beta>0$, onde $\Gamma^{\prime}$ é um contorno com as mesmas propriedades de $\Gamma$ á direita de $\Gamma$. Ainda mais,

$$
\left(A^{\alpha}\right)^{\beta}=\left[\left(A^{\alpha}\right)^{-\beta}\right]^{-1}=\left[A^{-\alpha \beta}\right]^{-1}=A^{\alpha \beta}
$$

para $\beta>0$. Isto prova o teorema. 


\subsection{Potências Imaginárias Limitadas}

Seja $E$ um espaço de Banach. Um operador linear $A$ em $E$ é dito ter potências imaginárias limitadas, em símbolos,

$$
A \in \mathcal{P} \mathcal{I} \mathcal{L}:=\mathcal{P} \mathcal{I} \mathcal{L}(E),
$$

se $A \in \mathcal{P}(E)$ e existe $\epsilon>0$ e $M \geq 1$ tal que

$$
A^{i t} \in L(E) \quad \text { e } \quad\left\|A^{i t}\right\|_{L(E)} \leq M, \quad-\epsilon \leq t \leq \epsilon .
$$

O Teorema a seguir mostra que estas hipóteses tem conseqüências muito interessantes.

Teorema 2.4.1 Suponha que $A \in \mathcal{P} \mathcal{I} \mathcal{L}$. Então $\left\{A^{z}: \operatorname{Re} z \leq 0\right\}$ é um semigrupo fortemente continuo sobre $L(E)$. Adicionalmente, $\left\{A^{i t}: t \in \mathbb{R}\right\}$ é um grupo fortemente contínuo sobre $E$ com gerador infinitesimal $i \log A$.

Prova. Se $|t| \in[n \epsilon,(n+1) \epsilon)$ para algum $n \in \mathbb{N}$, segue que

$$
\begin{aligned}
\left\|A^{-s+i t} x\right\| & \leq\left\|A^{-s}\left(A^{i \operatorname{sinal}(t) \epsilon}\right)^{n} A^{i \operatorname{sinal}(t)(|t|-n \epsilon)}\right\|\|x\| \\
& \leq\left\|A^{-s}\right\|\left\|\left(A^{i \operatorname{sinal}(t) \epsilon}\right)^{n}\right\|\left\|A^{i \operatorname{sinal}(t)(|t|-n \epsilon)}\right\|\|x\| \\
& \leq M^{m} M^{n} M\|x\| \leq M^{m} M\left(M^{n \epsilon}\right)^{\frac{1}{\epsilon}}\|x\| \\
& \leq M^{m} M\left(M^{|t|}\right)^{\frac{1}{\epsilon}}\|x\| \leq M^{m} M \mathbf{e}^{\theta|t|}\|x\|
\end{aligned}
$$

para $0 \leq s \leq m$ e $x \in D\left(A^{2 m}\right)$, onde $\theta=\epsilon^{-1} \log M \geq 0$. Portanto, da densidade de $D\left(A^{2 m}\right)$ em $E$, obtemos que

$$
\left\|A^{z}\right\| \leq M^{1-\operatorname{Re} z} \mathbf{e}^{\theta|\operatorname{Imz}|}, \operatorname{Re} z \leq 0 .
$$

Disto e do Teorema 2.2.3 (v) e (vii), segue que $z \mapsto A^{z}$ é um semigrupo fortemente contínuo em $\{z \in \mathbb{C}: \operatorname{Rez} \leq 0\}$. Agora utilizando o Teorema 2.2 .3 (iii) e a densidade de $D\left(A^{2 m}\right)$ em $E$, vemos que $\left\{A^{z}: \operatorname{Re} z \leq 0\right\}$ é um semigrupo fortemente contínuo em $E$. Conseqüêntemente, $\left\{A^{i t}: t \in \mathbb{R}\right\}$ é um grupo fortemente contínuo em $E$.

Resta mostrar que $i \log (A)$ é o gerador infinitesimal de $A^{i t}$. Para este fim, denote por $B$ o gerador infinitesimal deste grupo e recorde que

$$
B x=\lim _{t \rightarrow 0^{+}} \frac{A^{i t} x-x}{t}
$$

se e somente se $x \in D(B)$. Como

$$
\frac{A^{-s+i(t+\tau)} x-A^{-s+i t} x}{\tau}=A^{-s+i t} \frac{\left(A^{i \tau} x-x\right)}{\tau}
$$


para $x \in E, s \geq 0$ e $t, \tau \in \mathbb{R} \operatorname{com} \tau \neq 0$, vemos que

$$
B A^{-s+i t} x=A^{-s+i t} B x=\frac{d}{d t} A^{-s+i t} x
$$

par $x \in D(B), s \geq 0$ e $t \in \mathbb{R}$. Por outro lado, a analiticidade de $A^{-z}$ para $\operatorname{Re} z>0$ implica

$$
\frac{d}{d s} A^{-s+i t} x=i \frac{d}{d t} A^{-s+i t} x, x \in E, s>0, t \in \mathbb{R} .
$$

Como

$$
\frac{d}{d s} A^{-s+i t} x=\frac{d}{d s} A^{-s} A^{i t} x=-\log (A) A^{-s} A^{i t} x
$$

para $x \in E, s>0$, e $t \in \mathbb{R}$, graças a $\operatorname{Im}\left(A^{-s}\right) \subset D(\log (A))$, pelo Teorema 2.2.1 deduzimos de (2.33) e (2.34) que

$$
(i \log (A)) A^{-s+i t} x=B A^{-s+i t} x=A^{-s+i t} B x, s>0, t \in \mathbb{R},
$$

para $x \in D(B)$. Portanto, se $x \in D(B)$,

$$
(i \log (A)) A^{-s} x=B A^{-s} x=A^{-s} B x \rightarrow B x, \text { quando } s \rightarrow 0^{+} .
$$

Como $i \log (A)$ é fechado e $D(\log (A)) \ni A^{-s} x \rightarrow x$ quando $s \rightarrow 0^{+}$temos que $i \log (A) \supset B$. Por outro lado, o argumento usado em (2.33) implica

$$
B A^{-s+i t} x=\frac{d}{d t} A^{-s+i t} x, x \in E, s>0, t \in \mathbb{R}
$$

segue de (2.34) que, para $x \in D(\log (A))$,

$$
i B A^{-s} x=A^{-s}(-\log (A)) x \rightarrow-\log (A) x, s \rightarrow 0^{+} .
$$

Como $B$ é fechado e $D(B) \supset A^{-s} x \rightarrow x$ vemos que $i B x=-\log (A) x, x \in D(\log (A))$; isto é, $B \supset i \log (A)$. Isto prova o teorema.

Corolário 2.4.1 Suponha que $A \in \mathcal{P} \mathcal{I} \mathcal{L}$. Então existe uma constante $M \geq 1$ e $\theta \geq 0$ tal que

$$
\left\|A^{i t}\right\|_{L(E)} \leq M \mathbf{e}^{\theta|t|}, \quad t \in \mathbb{R}
$$

Prova. Segue da prova do teorema anterior fazendo $s=0 \mathrm{em}(2.32)$. 


\section{Capítulo 3}

\section{Potências Fracionárias de Operadores Acretivos}

O objetivo deste capítulo é responder a seguinte questão: Como mostrar que um determinado operador $A$ está em $\mathcal{P} \mathcal{I} \mathcal{L}$ ? Esta é uma questão central na caracterização dos espaços $E^{\alpha}$. Os teoremas que apresentaremos, devido a Kato [16, 17], mostram que em espaços de Hilbert, sempre que $A$ é gerador de um semigrupo fortemente contínuo com decaimento exponencial, $A$ tem potências imaginárias limitadas. Quando $E$ não é um espaço de Hilbert os resultados conhecidos são muito pouco abrangentes.

\subsection{Potências Fracionárias}

Definição 3.1.1 Diz-se que um operador $A$ é acretivo, se $-A$ é dissipativo. Se além disso, tivermos que $R(I+A)=H$, isto é, $\forall f \in H, \exists u \in D(A)$ tal que $u+A u=f$, dizemos que o operador $A$ é maximal acretivo.

Se $H$ é um espaço de Hilbert, suponha que $A: D(A) \subset H \rightarrow H$ seja maximal acretivo, do tipo $\left(\frac{\pi}{2}, M\right)$, ainda mais, que $A$ seja gerador de um semigrupo fortemente contínuo. É uma conseqüência direta da definição que $A+\epsilon$ é do tipo $\left(\frac{\pi}{2}, M\right)$ sempre que $A$ é do tipo $\left(\frac{\pi}{2}, M\right)$. Ainda, se $A$ é do tipo $\left(\frac{\pi}{2}, M\right)$, a potência fracionária $A^{\alpha}$ esta definida e é do tipo $\left(\frac{\pi \alpha}{2}, M\right)$ para $0 \leq \alpha \leq 1$. Em particular, $A^{\alpha}$ é também fechado e maximal acretivo e, portanto $-A^{\alpha}$ é o gerador infinitesimal de um semigrupo analítico $\left\{\mathbf{e}^{-t A^{\alpha}}: t \leq 0\right\}$ para $0 \leq \alpha<1$. Desde que $A^{*}$ é também fechado e maximal acretivo, $A^{* \alpha}$ está definido e goza de propriedades similares. Agora, definimos

$$
\mathcal{H}_{\alpha}=\frac{A^{\alpha}+A^{* \alpha}}{2}, \quad \mathcal{K}_{\alpha}=\frac{A^{\alpha}-A^{* \alpha}}{2 i}
$$


Lema 3.1.1 Assuma que $A$ é do tipo $\left(\frac{\pi}{2}, M\right)$ em um espaço de Hilbert $H$ e que $0<\alpha<1$. Para todo $\epsilon>0$ temos que $I+\epsilon A$ é também do tipo $\left(\frac{\pi}{2}, M\right)$ de forma que $(I+\epsilon A)^{\alpha}$ existe e

$$
\left\|(I+\epsilon A)^{-\alpha}\right\| \leq M
$$

Prova. Para ver que $(I+\epsilon A)$ é do tipo $\left(\frac{\pi}{2}, M\right)$ note que

$$
\begin{aligned}
\left\|(s+(I+\epsilon A))^{-1}\right\| & =\left\|(s+1+\epsilon A)^{-1}\right\|=\left\|\epsilon^{-1}\left((s+1) \epsilon^{-1}+A\right)^{-1}\right\| \\
& \leq \frac{M}{s+1} \leq \frac{M}{s} .
\end{aligned}
$$

Como $(I+\epsilon A)^{-1}$ é limitado, (2.27) vale para $\lambda=0$ se $A$ é substituído por $I+\epsilon A$. Como $\left\|(\mu+I+\epsilon A)^{-1}\right\| \leq M(\mu+1)^{-1}$, segue que

$$
\left\|(I+\epsilon A)^{-\alpha}\right\| \leq \frac{\operatorname{sen} \pi \alpha}{\pi} \int_{0}^{\infty} \mu^{-\alpha} M(\mu+1)^{-1} d \mu=M
$$

onde na última passagem usamos (2.11) com $E:=\mathbb{C}$ e $A=1$.

Lema 3.1.2 Se $H$ é um espaço de Hilbert, $A$ é do tipo $\left(\frac{\pi}{2}, 1\right)$ se e somente se $A$ é fechado e maximal acretivo.

Prova. Suponha que $A$ é do tipo $\left(\frac{\pi}{2}, 1\right)$, então $A$ é fechado e $\left\|\lambda(\lambda+A)^{-1}\right\| \leq 1$. Se $u \in D(A)$ então

$$
\|u\| \leq \lambda^{-1}\|(\lambda+A) u\|
$$

Elevando a desigualdade acima ao quadrado, segue

$$
\begin{aligned}
\|u\|^{2} & \leq \lambda^{-2}\|(\lambda+A) u\|^{2}=\lambda^{-2}\langle(\lambda+A) u,(\lambda+A) u\rangle \\
& =\lambda^{-2}\left(\|\lambda u\|^{2}+2 \lambda \operatorname{Re}\langle A u, u\rangle+\|A u\|^{2}\right) \\
& =\|u\|^{2}+2 \lambda^{-1} \operatorname{Re}\langle A u, u\rangle+\lambda^{-2}\|A u\|^{2} .
\end{aligned}
$$

Logo,

$$
0 \leq \lambda^{-1}\|A u\|^{2}+2 \operatorname{Re}\langle A u, u\rangle
$$

Agora, fazendo $\lambda \rightarrow \infty$, obtemos que

$$
\operatorname{Re}\langle A u, u\rangle \geq 0
$$

Por outro lado, assuma que $A$ seja fechado e maximal acretivo. Então, $\operatorname{Re}\langle A u, u\rangle \geq 0$, ou seja, $-A$ é dissipativo, do Lema 1.3.1, temos que

$$
\|\lambda+A\| \geq \lambda \text { isto é, } \quad\left\|\lambda(\lambda+A)^{-1}\right\| \leq 1
$$


Resta mostrar que $\overline{D(A)}=H, \rho(-A) \supset \operatorname{setor}\left\{\lambda \in \mathbb{C}:|\arg \lambda|<\frac{\pi}{2}\right\}$. Como $D(A)$ é a imagem de $(\lambda+A)^{-1}, \lambda>0$, é suficiente mostrar que $\left\langle(A+\lambda)^{-1} u, v\right\rangle=0, \forall u \in H \Rightarrow v=0$. Fazendo $u=v$ e $(\lambda+A)^{-1} v=w$, temos

$$
0=\operatorname{Re}\left\langle(\lambda+A)^{-1} v, v\right\rangle=\operatorname{Re}\langle w,(A+\lambda) w\rangle=\operatorname{Re}\langle w, A w\rangle+\operatorname{Re}\langle w, \lambda w\rangle \geq \lambda\|w\|^{2}
$$

e $\operatorname{assim} w=0, v=0$. E o resultado segue do Teorema de Lumer-Phillips.

Lema 3.1.3 Para todo $\epsilon>0$, temos que $D\left((A+\epsilon)^{\alpha}\right)=D\left(A^{\alpha}\right)$ e

$$
\left\|(A+\epsilon)^{\alpha} u-A^{\alpha} u\right\| \leq c \epsilon^{\alpha}\|u\|, \quad u \in D\left(A^{\alpha}\right)
$$

onde a constante $c$ depende somente de $\alpha$ e $M$.

Prova. Vamos denotar $A+\epsilon$ por $A_{\epsilon}$. Note que $A_{\epsilon}^{-\alpha} A_{\epsilon}^{-(1-\alpha)}=A_{\epsilon}^{-(1-\alpha)} A_{\epsilon}^{-\alpha}=A_{\epsilon}^{-1}$. Ainda, $A_{\epsilon}^{-1}, A_{\epsilon}^{-\alpha}$ são limitados para $\epsilon>0$. Segue que $A_{\epsilon}^{-(1-\alpha)} A_{\epsilon} \subset A_{\epsilon}^{\alpha}$. Agora, (2.27) é válido para $\lambda=0$, se $A$ é substituído por $A_{\epsilon}$. E mais, substituindo $\alpha$ por $1-\alpha$ e aplicando $A_{\epsilon} u$ a ambos os lados de (2.27), onde $u \in D(A)$, obtemos que

$$
A_{\epsilon}^{\alpha} u=\frac{\operatorname{sen} \pi \alpha}{\pi} \int_{0}^{\infty} u^{1-\alpha}\left(u+A_{\epsilon}\right)^{-1} A_{\epsilon} u d u, \quad \epsilon>0, u \in D(A) .
$$

Subtraindo de (3.3) uma expressão similar com $\epsilon$ substituído por $\eta, 0<\eta<\epsilon$, temos

$$
\begin{aligned}
A_{\epsilon}^{\alpha} u-A_{\eta}^{\alpha} u & =\frac{\operatorname{sen} \pi \alpha}{\pi}\left[\int_{0}^{\infty} u^{\alpha-1}\left(u+A_{\epsilon}\right)^{-1} A_{\epsilon} u d u-\int_{0}^{\infty} u^{\alpha-1}\left(u+A_{\eta}\right)^{-1} A_{\eta} u d u\right] \\
& =\frac{\operatorname{sen} \pi \alpha}{\pi}\left[\int_{0}^{\delta} u^{\alpha-1}\left(u+A_{\epsilon}\right)^{-1} A_{\epsilon} u d u-\int_{0}^{\delta} u^{\alpha-1}\left(u+A_{\eta}\right)^{-1} A_{\eta} u d u\right] \\
& +\frac{\operatorname{sen} \pi \alpha}{\pi}\left[\int_{\delta}^{\infty} u^{\alpha-1}\left(u+A_{\epsilon}\right)^{-1} A_{\epsilon} u d u-\int_{\delta}^{\infty} u^{\alpha-1}\left(u+A_{\eta}\right)^{-1} A_{\eta} u d u\right] \\
& =\frac{\operatorname{sen} \pi \alpha}{\pi}\left[\int_{0}^{\delta} u^{\alpha-1}\left(u+A_{\epsilon}\right)^{-1} A_{\epsilon} u d u-\int_{0}^{\delta} u^{\alpha-1}\left(u+A_{\eta}\right)^{-1} A_{\eta} u d u\right] \\
& +\frac{\operatorname{sen} \pi \alpha}{\pi}\left[\int_{\delta}^{\infty} u^{\alpha-1}\left(1-u\left(u+A_{\epsilon}\right)^{-1}\right) u d u-\int_{\delta}^{\infty} u^{\alpha-1}\left(1-u\left(u+A_{\eta}\right)^{-1}\right) u d u\right] \\
& =\frac{\operatorname{sen} \pi \alpha}{\pi}\left[\int_{0}^{\infty} u^{\alpha-1}\left(u+A_{\epsilon}\right)^{-1} A_{\epsilon} u d u-\int_{0}^{\infty} u^{\alpha-1}\left(u+A_{\eta}\right)^{-1} A_{\eta} u d u\right] \\
& +\frac{\operatorname{sen} \pi \alpha}{\pi}\left[\int_{\delta}^{\infty} u^{\alpha}\left(\left(u+A_{\eta}\right)^{-1}-\left(u+A_{\epsilon}\right)^{-1}\right) u d u\right] \\
& =\frac{\operatorname{sen} \pi \alpha}{\pi}\left[\int_{0}^{\delta} u^{\alpha-1}\left(u+A_{\epsilon}\right)^{-1} A_{\epsilon} u d u-\int_{0}^{\delta} u^{\alpha-1}\left(u+A_{\eta}\right)^{-1} A_{\eta} u d u\right] \\
& +\frac{\operatorname{sen} \pi \alpha}{\pi}\left[(\epsilon-\eta) \int_{\delta}^{\infty} u^{\alpha}\left(u+A_{\epsilon}\right)^{-1}\left(u+A_{\eta}\right)^{-1} u d u\right] .
\end{aligned}
$$


Como $\left\|\left(u+A_{\epsilon}\right)^{-1} A_{\epsilon} u\right\| \leq(1+M)\|u\|$ e $\left\|\left(u+A_{\epsilon}\right)^{-1}\right\| \leq M u^{-1}$, segue que

$$
\begin{aligned}
\left\|A_{\epsilon}^{-\alpha} u-A_{\eta}^{\alpha} u\right\| & \leq \frac{\operatorname{sen} \pi \alpha}{\pi}\left[\int_{0}^{\delta} 2(1+M) u^{\alpha-1}\|u\| d u+(\epsilon-\eta) M^{2} \int_{\delta}^{\infty} u^{\alpha-2}\|u\| d u\right] \\
& =\frac{\operatorname{sen} \pi \alpha}{\pi}\left[2(1+M) \frac{\delta^{\alpha}}{\alpha}\|u\|+(\epsilon-\eta) M^{2}\|u\| \frac{\delta^{\alpha-1}}{1-\alpha}\right] .
\end{aligned}
$$

Tomando $\delta=\frac{(\epsilon-\eta) M^{2}}{2(1+M)}$, obtemos

$$
\begin{aligned}
\left\|A_{\epsilon}^{-\alpha} u-A_{\eta}^{\alpha} u\right\| & \leq \frac{\operatorname{sen} \pi \alpha}{\pi}\left[\frac{(\epsilon-\eta)^{\alpha} M^{2 \alpha}}{2^{\alpha} \alpha(1+M)^{\alpha}} 2(1+M)+\frac{(\epsilon-\eta) M^{2}(\epsilon-\eta)^{\alpha-1} M^{2 \alpha-2}}{(1-\alpha) 2^{\alpha-1}(1+M)^{\alpha-1}}\right]\|u\| \\
& =\frac{\operatorname{sen} \pi \alpha}{\pi}\left[\frac{(\epsilon-\eta)^{\alpha} M^{2 \alpha}}{\alpha(2(1+M))^{\alpha-1}}+\frac{(\epsilon-\eta)^{\alpha} M^{2 \alpha}}{(1-\alpha)(2(1+M))^{\alpha-1}}\right]\|u\| \\
& =\frac{\operatorname{sen} \pi \alpha}{\pi} \frac{(\epsilon-\eta)^{\alpha} M^{2 \alpha}}{(2(1+M))^{\alpha-1}}\left[\frac{1}{\alpha(1-\alpha)}\right]\|u\| \\
& =c(\epsilon-\eta)^{\alpha}\|u\|,
\end{aligned}
$$

onde $c=\frac{\operatorname{sen} \pi \alpha}{\pi} \frac{M^{2 \alpha}}{(2(1+M))^{\alpha-1} \alpha(1-\alpha)}$ depende somente de $\alpha$ e $M$. Isto mostra que $\lim _{\epsilon \rightarrow 0} A_{\epsilon}^{\alpha} u$ existe para $u \in D(A)$, denotemos por $B u$ tal limite, e assim

$$
\left\|A_{\epsilon}^{-\alpha} u-B u\right\| \leq c \epsilon^{\alpha}\|u\|, \quad \epsilon>0, \quad u \in D(A) .
$$

Agora, $D(A)$ é um principal de $A_{\epsilon}^{\alpha}$, no sentido de que o fecho da restrição de $A_{\epsilon}^{\alpha}$ ao $D(A)$ é o próprio $A_{\epsilon}^{\alpha}$. Para ver isto, é suficiente observar que, para $v \in D\left(A_{\epsilon}^{\alpha}\right)$, a seqüência

$$
v_{n}=\left(1+n^{-1} A_{\epsilon}\right)^{-1} v \in D\left(A_{\epsilon}\right)=D(A)
$$

tem a seguinte propriedade

$$
v_{n} \longrightarrow v \quad \text { e } \quad A_{\epsilon}^{\alpha} v_{n}=\left(1+n^{-1} A_{\epsilon}\right)^{-1} A_{\epsilon}^{\alpha} v \longrightarrow A_{\epsilon}^{\alpha} v, \quad n \rightarrow \infty .
$$

Aqui usamos a relação

$$
A_{\epsilon}^{\alpha}\left(1+n^{-1} A_{\epsilon}\right)^{-1} v=\left(1+n^{-1} A_{\epsilon}\right)^{-1} A_{\epsilon}^{\alpha} v .
$$

Isto segue de

$$
\left(1+n^{-1} A_{\epsilon}\right)^{-1} A_{\epsilon}^{-\alpha} w=A_{\epsilon}^{-\alpha}\left(1+n^{-1} A_{\epsilon}\right)^{-1} w, \quad \text { com } \quad w=A_{\epsilon}^{\alpha} v
$$

Como $A$ é gerador de um semigrupo fortemente contínuo, o fato de $D(A)$ ser um principal de $A_{\epsilon}^{\alpha}$, combinado com (3.4), resulta para $u \in D(A)$ que

$$
\|(\lambda-B) u\|=\left\|\left(\lambda-A_{\epsilon}^{\alpha}\right) u+\left(A_{\epsilon}^{\alpha}-B\right) u\right\| \geq\|\|\left(\lambda-A_{\epsilon}^{\alpha}\right) u\|-\|\left(A_{\epsilon}^{\alpha}-B\right) u\|\| \geq \lambda\|u\|-c \epsilon^{c}\|u\|
$$


fazendo $\epsilon \rightarrow 0$, obtemos que $B$ é dissipativo. Então do Teorema 1.3.3, segue que $B$ é fechável, ou seja, o fecho $B^{* *}$ de $B$ existe ( $B$ está definido somente sobre $D(A)$ ) e tem o mesmo domínio que $A_{\epsilon}^{\alpha}$. Ao mesmo tempo, a desigualdade (3.4) é estendida para todo $u \in D\left(B^{* *}\right)$, com $B$ substituído por $B^{* *}$. Então $D\left(A_{\epsilon}^{\alpha}\right)=D\left(B^{* *}\right)$ é independente de $\epsilon$ e $A_{\epsilon}^{\alpha} u \longrightarrow B^{* *} u$ uniformemente para $\|u\| \leq 1, u \in D\left(B^{* *}\right)$. Isto mostra que $\left(B^{* *}+\lambda\right)^{-1}$ existe como um operador limitado com domínio $H$ para $\lambda>0$ e que $\left(A_{\epsilon}^{\alpha}+\lambda\right)^{-1} \longrightarrow\left(B^{* *}+\lambda\right)^{-1}$ para $\epsilon \rightarrow 0$ na topologia uniforme. Como sabemos que $\left(A_{\epsilon}^{\alpha}+\lambda\right)^{-1} \longrightarrow\left(A^{\alpha}+\lambda\right)^{-1}$, segue que $B^{* *}=A^{\alpha}$.

Lema 3.1.4 $D(A)$ é um principal de $A^{\alpha}$.

Prova. Segue da prova do Lema 3.1.3, pois $A^{\alpha}=B^{* *}$ é o fecho de $B$ e $B$ é a restrição de $B^{* *}=A^{\alpha}$ a $D(A)$.

Lema 3.1.5 Para cada $u \in D(A), 0<\alpha<1$, temos que

$$
A^{\alpha} u=\frac{\operatorname{sen} \pi \alpha}{\pi} \int_{0}^{\infty} \lambda^{\alpha-1} A(\lambda+A)^{-1} u d \lambda
$$

a integral é absolutamente convergente.

Prova. Note que

$$
\int_{0}^{\infty} \lambda^{\alpha-1} A(\lambda+A)^{-1} u d \lambda=\int_{0}^{1} \lambda^{\alpha-1} A(\lambda+A)^{-1} u d \lambda+\int_{1}^{\infty} \lambda^{\alpha-1} A(\lambda+A)^{-1} u d \lambda
$$

ainda, $\left\|A(A+\lambda)^{-1}\right\| \leq 1+M$ e para $u \in D(A)$

$$
\left\|A(A+\lambda)^{-1} u\right\| \leq\left\|(A+\lambda)^{-1} A u\right\| \leq \frac{M}{\lambda}\|A u\|
$$

assim,

$$
\begin{aligned}
\left\|\int_{0}^{\infty} \lambda^{\alpha-1} A(\lambda+A)^{-1} u d \lambda\right\| & \leq M\|u\| \int_{0}^{1} \lambda^{\alpha-1} d \lambda+M\|A u\| \int_{1}^{\infty} \lambda^{\alpha-2} d \lambda \\
& =\frac{M\|u\|}{\alpha}-\frac{M\|A u\|}{\alpha-1} .
\end{aligned}
$$

Sabemos que (3.5) é válido para $A$ substituído por $A_{\epsilon}=A+\epsilon$. Então (3.5) segue por (3.3), fazendo $\epsilon \rightarrow 0$, observando que $A_{\epsilon}\left(\lambda+A_{\epsilon}\right)^{-1} u \rightarrow A(\lambda+A)^{-1} u$.

Lema 3.1.6 Suponha que A é um operador fechado, maximal acretivo em um espaço de Hilbert $H$ e que $\operatorname{Re}\langle A u, u\rangle \geq \delta\langle u, u\rangle$ para algum $\delta>0$ e todo $u \in D(A)$. Então $\operatorname{Re}\left\langle A^{\alpha} u, u\right\rangle \geq \delta^{\alpha}\langle u, u\rangle$, para todo $u \in D\left(A^{\alpha}\right)$. 
Prova. É suficiente provar a afirmativa para $u \in D(A)$, pois $D(A)$ é um principal de $A^{\alpha}$ e, portanto, o resultado pode ser estendido para todo $u \in D\left(A^{\alpha}\right)$ por continuidade. Se $u \in D(A)$, temos a expressão (3.5) para $A^{\alpha} u$. Então é suficiente mostrar que

$$
\operatorname{Re}\left\langle A(\lambda+A)^{-1} u, u\right\rangle \geq \delta(\lambda+\delta)^{-1}\langle u, u\rangle
$$

Como

$$
\left\langle A^{\alpha} u, u\right\rangle=\frac{\operatorname{sen} \pi \alpha}{\pi} \int_{0}^{\infty} \lambda^{\alpha-1}\left\langle A(\lambda+A)^{-1} u, u\right\rangle d \lambda
$$

Note que,

$$
\begin{aligned}
\operatorname{Re}\langle(\lambda+A) u, u\rangle & =\operatorname{Re}\langle\lambda u, u\rangle+\operatorname{Re}\langle A u, u\rangle=\lambda \operatorname{Re}\langle u, u\rangle+\operatorname{Re}\langle A u, u\rangle \\
& =\lambda\langle u, u\rangle+\operatorname{Re}\langle A u, u\rangle \geq \lambda\langle u, u\rangle+\delta\langle u, u\rangle=(\lambda+\delta)\langle u, u\rangle
\end{aligned}
$$

$\log \mathrm{O}$

$$
\operatorname{Re}\left\langle(\lambda+A)^{-1} u, u\right\rangle \leq(\lambda+\delta)^{-1}\langle u, u\rangle
$$

e, como $A(\lambda+A)^{-1}=1-\lambda(\lambda+A)^{-1}$, obtemos que

$$
\begin{aligned}
\operatorname{Re}\left\langle A(\lambda+A)^{-1} u, u\right\rangle & =\operatorname{Re}\langle u, u\rangle-\lambda \operatorname{Re}\left\langle(\lambda+A)^{-1} u, u\right\rangle=\langle u, u\rangle-\lambda \operatorname{Re}\left\langle(\lambda+A)^{-1} u, u\right\rangle \\
& \geq\langle u, u\rangle-\lambda(\lambda+\delta)^{-1}\langle u, u\rangle=\left(1-\lambda(\lambda+\delta)^{-1}\right)\langle u, u\rangle \\
& =\delta(\lambda+\delta)^{-1}\langle u, u\rangle .
\end{aligned}
$$

Assim,

$$
\begin{aligned}
\operatorname{Re}\left\langle A^{\alpha} u, u\right\rangle & =\frac{\operatorname{sen} \pi \alpha}{\pi} \int_{0}^{\infty} \lambda^{\alpha-1} \operatorname{Re}\left\langle A(\lambda+A)^{-1} u, u\right\rangle d \lambda \\
& \geq \frac{\operatorname{sen} \pi \alpha}{\pi} \int_{0}^{\infty} \lambda^{\alpha-1} \delta(\lambda+\delta)^{-1}\langle u, u\rangle d \lambda \\
& =\frac{\operatorname{sen} \pi \alpha}{\pi} \int_{0}^{\infty} \frac{\lambda^{-(1-\alpha)}}{\lambda+\delta}\langle u, u\rangle d \lambda \quad 0<\operatorname{Re}(1-\alpha)<1 \\
& =\delta \delta^{\alpha-1}\langle u, u\rangle=\delta^{\alpha}\langle u, u\rangle .
\end{aligned}
$$

Teorema 3.1.1 Seja $H$ um espaço de Hilbert e $A: D(A) \subset H \rightarrow H$ um operador fechado, densamente definido e maximal acretivo com $0 \in \rho(A)$, então, para $0 \leq \alpha \leq \frac{1}{2}$

$$
D\left(A^{\alpha}\right)=D\left(A^{* \alpha}\right)=D\left(\mathcal{H}_{\alpha}\right)=D\left(\mathcal{K}_{\alpha}\right)=D_{\alpha}
$$

$\mathcal{H}_{\alpha}$ é auto-adjunto e não negativo, $\mathcal{K}_{\alpha}$ é anti-simétrico e para todo $u \in D_{\alpha}$

1. $\left\|K_{\alpha} u\right\| \leq \tan \frac{\pi \alpha}{2}\left\|\mathcal{H}_{\alpha} u\right\|$,

2. $\left(1-\tan \frac{\pi \alpha}{2}\right)\left\|\mathcal{H}_{\alpha} u\right\| \leq\left\|A^{\alpha} u\right\| \leq\left(1+\tan \frac{\pi \alpha}{2}\right)\left\|\mathcal{H}_{\alpha} u\right\|$ 
3. $\left\|A^{* \alpha} u\right\| \leq \tan \frac{\pi(1+2 \alpha)}{4}\left\|A^{\alpha} u\right\|$

4. $\operatorname{Re}\left\langle A^{\alpha} u, A^{* \alpha} u\right\rangle \geq \cos \pi \alpha\left\|A^{\alpha} u\right\|\left\|A^{* \alpha} u\right\|$

5. $\operatorname{Re}\left\langle A^{\alpha} u, \mathcal{H}_{\alpha} u\right\rangle \geq \frac{(\cos \pi \alpha)^{\frac{1}{2}}}{\cos \frac{\pi \alpha}{2}}\left\|A^{\alpha} u\right\|\left\|\mathcal{H}_{\alpha} u\right\|$.

$O$ mesmo vale quando trocamos $A^{\alpha}$ por $A^{* \alpha}$.

Prova. Passo1. Primeiramente assuma que $A$ é limitado e $\operatorname{Re}\langle A u, u\rangle \geq \delta\langle u, u\rangle, \delta>0$ e $A^{-1} \in L(H)$. Então $A^{\alpha}$ está definido para todo número complexo $\alpha$ por

$$
A^{\alpha}=\frac{1}{2 \pi i} \int_{C} \lambda^{\alpha}(\lambda-A)^{-1} d \lambda
$$

onde $C$ é uma curva fechada, retificável e simples evitando o semi-eixo real negativo e o zero. Tal curva $C$ pode ser encontrada, pois $0 \in \rho(A)$. Pela definição de $A^{\alpha}$ acima, segue que $A^{\alpha}$ é uma função inteira de $\alpha$, da mesma forma $A^{* \alpha}$ é uma função inteira de $\alpha$ e o mesmo vale para $\mathcal{H}_{\alpha}$ e para $\mathcal{K}_{\alpha}$. Daí

$$
\begin{aligned}
\left\|\mathcal{H}_{\alpha} u\right\|^{2}-\left\|\mathcal{K}_{\alpha}\right\|^{2} & =\left\langle\frac{A^{\alpha}+A^{* \alpha}}{2}, \frac{A^{\alpha}+A^{* \alpha}}{2}\right\rangle+\left\langle\frac{A^{\alpha}-A^{* \alpha}}{2 i}, \frac{A^{\alpha}-A^{* \alpha}}{2 i}\right\rangle \\
& =\frac{1}{4}\left\langle A^{\alpha}+A^{* \alpha}, A^{\alpha}+A^{* \alpha}\right\rangle+\frac{1}{2 i} \frac{1}{2 i}\left\langle A^{\alpha}-A^{* \alpha}, A^{\alpha}-A^{* \alpha}\right\rangle \\
& =\frac{1}{4}\left(\left\|A^{\alpha}\right\|^{2}+\left\langle A^{\alpha}, A^{* \alpha}\right\rangle+\left\langle A^{* \alpha, A^{\alpha}}\right\rangle\left\|A^{* \alpha}\right\|^{2}\right) \\
& -\frac{1}{4}\left(\left\|A^{\alpha}\right\|^{2}-\left\langle A^{\alpha}, A^{* \alpha}\right\rangle-\left\langle A^{* \alpha}, A^{\alpha}\right\rangle+\left\|A^{* \alpha}\right\|\right) \\
& =\operatorname{Re}\left\langle A^{\alpha} u, A^{* \alpha} u\right\rangle=\operatorname{Re}\left\langle A^{\alpha+\bar{\alpha}} u, u\right\rangle
\end{aligned}
$$

onde a última igualdade segue do fato que $A^{* \alpha *}=A^{\bar{\alpha}}$ e esta igualdade é obtida da seguinte forma: Para todo $u, v \in H$ e $C$ simétrica relativamente ao eixo real temos

$$
\begin{aligned}
\left\langle u, A^{* \alpha} v\right\rangle & =\left\langle u, \frac{1}{2 \pi i} \int_{C} \lambda^{\alpha}\left(\lambda-A^{*}\right)^{-1} d \lambda v\right\rangle=\int_{C}\left\langle u, \frac{1}{2 \pi i} \lambda^{\alpha}\left(\lambda-A^{*}\right)^{-1} v\right\rangle d \bar{\lambda} \\
& =\int_{C}\left\langle-\frac{1}{2 \pi i} \bar{\lambda}^{\bar{\alpha}}\left(\left(\lambda-A^{*}\right)^{-1}\right)^{*} u, v\right\rangle d \bar{\lambda}=\left\langle\int_{-C} \frac{1}{2 \pi i} \bar{\lambda}^{\bar{\alpha}}\left((\bar{\lambda}-A)^{-1}\right) u d \bar{\lambda}, v\right\rangle \\
& =\left\langle A^{\bar{\alpha}} u, v\right\rangle
\end{aligned}
$$

(na última integral a mudança de $\lambda$ para $\bar{\lambda}$ inverte a orientação da curva) e $A^{* \alpha *}=A^{\bar{\alpha}}$. Segue de (3.7) que

$$
\left\|\mathcal{K}_{\alpha}\right\| \leq\left\|\mathcal{H}_{\alpha}\right\|, \quad-\frac{1}{2} \leq \operatorname{Re} \alpha \leq \frac{1}{2},
$$

isto é óbvio para $0 \leq \operatorname{Re} \alpha \leq \frac{1}{2}$, pois $A^{\beta}$ é acretivo se $0 \leq \beta \leq 1$ enquanto para $-\frac{1}{2} \leq \operatorname{Re} \alpha \leq 0$ é suficiente mostrar que $A^{-1}$ é acretivo, e isto segue de

$$
\operatorname{Re}\left\langle A^{-1} u, u\right\rangle=\operatorname{Re}\left\langle A^{-1} u, A A^{-1} u\right\rangle \geq \delta\left\|A^{-1} u\right\|^{2} \geq \delta\|A\|^{-2}\|u\|^{2} \geq 0 .
$$


De (3.7) obtemos

$$
\left\|\mathcal{H}_{\alpha} u\right\|^{2} \geq \operatorname{Re}\left\langle A^{2 \xi} u, u\right\rangle \geq \delta^{2 \xi}\|u\|^{2}, \quad 0 \leq \xi \leq \frac{1}{2}, \quad \xi=\operatorname{Re} \alpha
$$

e de (3.9) temos que

$$
\begin{aligned}
\left\|\mathcal{H}_{\alpha} u\right\|^{2} & \geq \operatorname{Re}\left\langle A^{-2|\xi|} u, u\right\rangle=\operatorname{Re}\left\langle A^{-2|\xi|} u, A^{-2|\xi|} A^{2|\xi|} u\right\rangle \geq \delta^{2|\xi|}\left\|A^{-2|\xi|} u\right\|^{2} \\
& \geq \delta^{2|\xi|}\left\|A^{-2|\xi|}\right\|^{2}\|u\|^{2} \geq\left(\delta\|A\|^{-2}\right)^{2|\xi|}\|u\|^{2}, \quad-\frac{1}{2} \leq \xi \leq 0 .
\end{aligned}
$$

Estas desigualdades mostram que $\mathcal{H}_{\alpha}$ tem inversa limitada $\mathcal{H}_{\alpha}^{-1}$ para $|\operatorname{Re} \alpha| \leq \frac{1}{2}$. O domínio de $\mathcal{H}_{\alpha}^{-1}$ é $H$ para $\alpha \in \mathbb{R}$ pois $\mathcal{H}_{\alpha}$ é auto-adjunto (auto-adjunto e coercivo é sobre). Como $\mathcal{H}_{\alpha}$ é contínuo em $\alpha$ segue que $\mathcal{H}_{\alpha}$ tem domínio $H$ para todo $\alpha \operatorname{com}|\operatorname{Re} \alpha| \leq \frac{1}{2}$. E com isto (3.8) é equivalente a

$$
\left\|\mathcal{K}_{\alpha} \mathcal{H}_{\alpha}^{-1}\right\| \leq 1, \quad|\operatorname{Re} \alpha| \leq \frac{1}{2}
$$

Agora considere a função

$$
T(\alpha)=\frac{1}{\tan \frac{\pi \alpha}{2}} \mathcal{K}_{\alpha} \mathcal{H}_{\alpha}^{-1}
$$

$T(\alpha)$ é uma função analítica na faixa $|\operatorname{Re} \alpha| \leq \frac{1}{2}$ pois $\mathcal{K}_{\alpha}$ tem um zero em $\alpha=0$ (singularidade removível em $\alpha=0$ ). Observe que $\left|\tan \frac{\pi \alpha}{2}\right|=1$ para $\alpha$ na fronteira da faixa. De fato, lembre que $\tan \theta=\frac{1}{i} \frac{\mathbf{e}^{i \theta}-\mathbf{e}^{-i \theta}}{\mathbf{e}^{i \theta}+\mathbf{e}^{i \theta}}$, e assim

$$
\left|\tan \frac{\pi \alpha}{2}\right|=\left|\frac{\mathbf{e}^{i \frac{\pi \alpha}{2}}-\mathbf{e}^{-i \frac{\pi \alpha}{2}}}{\mathbf{e}^{i \frac{\pi \alpha}{2}}+\mathbf{e}^{-i \frac{\pi \alpha}{2}}}\right|=\frac{\left|\mathbf{e}^{i \pi \alpha}-1\right|}{\left|\mathbf{e}^{i \pi \alpha+1}\right|}
$$

Por outro lado,

$$
\overline{\mathbf{e}^{i \pi \alpha}}=\mathbf{e}^{-i \pi \bar{\alpha}}=\mathbf{e}^{-\pi(i \operatorname{Re} \alpha+\operatorname{Im} \alpha)}
$$

Agora, para $\operatorname{Re} \alpha= \pm \frac{1}{2}$, temos

$$
\overline{\mathbf{e}^{i \pi \alpha}}=\mathbf{e}^{-i \frac{\pi}{2}} \mathbf{e}^{-\pi \operatorname{Im} \alpha}=-i \mathbf{e}^{-\pi \operatorname{Im} \alpha} \quad \text { e } \overline{\mathbf{e}^{i \pi \alpha}}=\mathbf{e}^{i \frac{\pi}{2}} \mathbf{e}^{-\pi \operatorname{Im} \alpha}=i \mathbf{e}^{-\pi \operatorname{Im} \alpha}
$$

Portanto,

$$
\overline{\mathbf{e}^{i \pi \alpha}}=-\mathbf{e}^{i \pi \alpha}, \quad \text { para } \quad \operatorname{Re} \alpha= \pm \frac{1}{2},
$$

e conseqüêntemente,

$$
\left|\tan \frac{\pi \alpha}{2}\right|=\frac{\left|\mathbf{e}^{i \pi \alpha}-1\right|}{\left|\mathbf{e}^{i \pi \alpha}+1\right|}=\frac{\left|\overline{\mathbf{e}^{i \pi \alpha}}-1\right|}{\left|\mathbf{e}^{i \pi \alpha}+1\right|}=\frac{\left|-\mathbf{e}^{i \pi \alpha}-1\right|}{\left|\mathbf{e}^{i \pi \alpha}+1\right|}=1, \quad \operatorname{Re} \alpha= \pm \frac{1}{2} .
$$

Restringindo $\alpha$ a $0 \leq \alpha \leq \frac{1}{2}$ temos que (1) vale e mais

$$
\left\|\mathcal{K}_{\alpha} \mathcal{H}_{\alpha}^{-1}\right\| \leq\left|\tan \frac{\pi \alpha}{2}\right|, \quad|\operatorname{Re} \alpha| \leq \frac{1}{2}
$$


e

$$
\left\|\mathcal{K}_{\alpha} u\right\| \leq\left|\tan \frac{\pi \alpha}{2}\right|\left\|\mathcal{H}_{\alpha} u\right\|, \quad u \in H, \quad|\operatorname{Re} \alpha| \leq \frac{1}{2} .
$$

Para mostrar (2), seja $0 \leq \alpha \leq \frac{1}{2}$, note que $A^{\alpha}=\mathcal{H}_{\alpha}+i \mathcal{K}_{\alpha}$ e use (1). De fato,

$$
\begin{aligned}
\left\|A^{\alpha} u\right\| & =\left\|\mathcal{H}_{\alpha} u+i \mathcal{K}_{\alpha} u\right\| \leq\left\|\mathcal{H}_{\alpha} u\right\|+\left\|\mathcal{K}_{\alpha} u\right\| \\
& \leq\left\|\mathcal{H}_{\alpha} u\right\|+\left|\tan \frac{\pi \alpha}{2}\right|\left\|\mathcal{H}_{\alpha} u\right\|=\left(1+\tan \frac{\pi \alpha}{2}\right)\left\|\mathcal{H}_{\alpha} u\right\|
\end{aligned}
$$

Por outro lado,

$$
\begin{aligned}
\left\|A^{\alpha} u\right\| & =\left\|\mathcal{H}_{\alpha} u+i \mathcal{K}_{\alpha} u\right\| \geq\left|\left\|\mathcal{H}_{\alpha} u\right\|-\left\|i \mathcal{K}_{\alpha} u\right\|\right|=\left|\left\|\mathcal{H}_{\alpha} u\right\|-\left\|\mathcal{K}_{\alpha} u\right\|\right| \\
& =\left\|\mathcal{H}_{\alpha} u\right\|-\left\|\mathcal{K}_{\alpha} u\right\| \geq\left\|\mathcal{H}_{\alpha}\right\|-\tan \frac{\pi \alpha}{2}\left\|\mathcal{H}_{\alpha} u\right\| \\
& =\left(1-\tan \frac{\pi \alpha}{2}\right)\left\|\mathcal{H}_{\alpha} u\right\|
\end{aligned}
$$

Para mostrar (3), observe que

$$
\frac{1+\tan \frac{\pi \alpha}{2}}{1-\tan \frac{\pi \alpha}{2}}=\tan \frac{\pi(1+2 \alpha)}{4}
$$

Note que (2) é válido, substituindo $A$ por $A^{*}$, então

$$
\left(1-\tan \frac{\pi \alpha}{2}\right)\left\|\mathcal{H}_{\alpha} u\right\| \leq\left\|A^{* \alpha} u\right\| \leq\left(1+\tan \frac{\pi \alpha}{2}\right)\left\|\mathcal{H}_{\alpha} u\right\|
$$

segue que

$$
1 \leq \frac{\left\|A^{* \alpha} u\right\|}{\left(1-\tan \frac{\pi \alpha}{2}\right)\left\|\mathcal{H}_{\alpha} u\right\|} \leq \frac{1+\tan \frac{\pi \alpha}{2}}{1-\tan \frac{\pi \alpha}{2}}=\tan \frac{\pi(1+2 \alpha)}{4}
$$

e assim

$$
\left\|A^{* \alpha} u\right\| \leq \tan \frac{\pi(1+2 \alpha)}{4}\left(1-\tan \frac{\pi \alpha}{2}\right)\left\|\mathcal{H}_{\alpha} u\right\|
$$

o que resulta

$$
\left\|A^{* \alpha} u\right\| \leq \tan \frac{\pi(1+2 \alpha)}{4}\left\|A^{\alpha} u\right\|
$$

Para provar (4) substituímos $\mathcal{H}_{\alpha}=\left(A^{\alpha}+A^{* \alpha}\right) / 2$ e $\mathcal{K}_{\alpha}=\left(A^{\alpha}-A^{* \alpha}\right) /(2 i)$ em (1) para obter

$$
\tan \frac{\pi \alpha}{2}\left\|\left(A^{\alpha}+A^{* \alpha}\right) u\right\| \leq\left\|\left(A^{\alpha}-A^{* \alpha}\right) u\right\|
$$

Elevando a expressão acima ao quadrado e simplificando, obtemos

$$
\begin{gathered}
\left\|A^{\alpha}-A^{* \alpha}\right\|^{2} \leq \tan ^{2} \frac{\pi \alpha}{2}\left\|A^{\alpha}+A^{* \alpha}\right\|^{2} \Rightarrow \\
\left\langle A^{\alpha}-A^{* \alpha}, A^{\alpha}-A^{* \alpha}\right\rangle \leq \tan ^{2} \frac{\pi \alpha}{2}\left(\left\langle A^{\alpha}+A^{* \alpha}, A^{\alpha}+A^{* \alpha}\right\rangle\right) \Rightarrow \\
\left\|A^{\alpha}\right\|^{2}-2 \operatorname{Re}\left\langle A^{\alpha}, A^{* \alpha}\right\rangle+\left\|A^{* \alpha}\right\|^{2} \leq \tan ^{2} \frac{\pi \alpha}{2}\left(\left\|A^{\alpha}\right\|^{2}+2 \operatorname{Re}\left\langle A^{\alpha}, A^{* \alpha}\right\rangle+\left\|A^{* \alpha}\right\|^{2}\right) \Rightarrow \\
-2 \operatorname{Re}\left\langle A^{\alpha}, A^{* \alpha}\right\rangle \leq\left(\tan ^{2} \frac{\pi \alpha}{2}-1\right)\left(\left\|A^{\alpha}\right\|^{2}+\left\|A^{* \alpha}\right\|^{2}\right)+\tan ^{2} \frac{\pi \alpha}{2}\left(2 \operatorname{Re}\left\langle A^{\alpha}, A^{* \alpha}\right\rangle\right) \Rightarrow
\end{gathered}
$$




$$
\begin{gathered}
-\left(1+\tan ^{2} \frac{\pi \alpha}{2}\right) 2 \operatorname{Re}\left\langle A^{\alpha}, A^{* \alpha}\right\rangle \leq\left(\frac{\operatorname{sen}^{2} \frac{\pi \alpha}{2}}{\cos ^{2} \frac{\pi \alpha}{2}}-1\right)\left(\left\|A^{\alpha}\right\|^{2}+\left\|A^{* \alpha}\right\|^{2}\right) \Rightarrow \\
-\left(\sec ^{2} \frac{\pi \alpha}{2}\right) 2 \operatorname{Re}\left\langle A^{\alpha, A^{* \alpha}}\right\rangle \leq-\frac{\left(\cos ^{2} \frac{\pi \alpha}{2}-\operatorname{sen}^{2} \frac{\pi \alpha}{2}\right)}{\cos ^{2} \frac{\pi \alpha}{2}}\left(\left\|A^{\alpha}\right\|^{2}+\left\|A^{* \alpha}\right\|^{2}\right) \Rightarrow \\
2 \operatorname{Re}\left\langle A^{\alpha}, A^{* \alpha}\right\rangle \geq \cos \pi \alpha\left(\left\|A^{\alpha}\right\|^{2}+\left\|A^{* \alpha}\right\|^{2}\right)
\end{gathered}
$$

Assim,

$$
2 \operatorname{Re}\left\langle A^{\alpha}, A^{* \alpha}\right\rangle \geq \cos \pi \alpha\left(\left\|A^{\alpha}\right\|^{2}+\left\|A^{* \alpha}\right\|^{2}\right) \geq 2 \cos \pi \alpha\left\|A^{\alpha}\right\|\left\|A^{* \alpha}\right\|
$$

e, portanto

$$
\operatorname{Re}\left\langle A^{\alpha}, A^{* \alpha}\right\rangle \geq \cos \pi \alpha\left\|A^{\alpha}\right\|\left\|A^{* \alpha}\right\|
$$

o que prova (4). A prova de (5) é obtida substituindo $i \mathcal{K}_{\alpha}=A^{\alpha}-\mathcal{H}_{\alpha}$ em (1) o que nos dá

$$
\left\|A^{\alpha} u-\mathcal{H}_{\alpha} u\right\| \leq \tan \frac{\pi \alpha}{2}\left\|\mathcal{H}_{\alpha} u\right\|
$$

que quando elevada ao quadrado, resulta

$$
\left\|A^{\alpha} u\right\|^{2}-\left\langle A^{\alpha} u, \mathcal{H}_{\alpha} u\right\rangle-\left\langle\mathcal{H}_{\alpha} u, A^{\alpha} u\right\rangle+\left\|\mathcal{H}_{\alpha} u\right\|^{2} \leq \tan ^{2} \frac{\pi \alpha}{2}\left\|\mathcal{H}_{\alpha} u\right\|^{2}
$$

de onde segue que

$$
\begin{aligned}
2 \operatorname{Re}\left\langle A^{\alpha} u, \mathcal{H}_{\alpha} u\right\rangle & \geq\left(1-\tan ^{2} \frac{\pi \alpha}{2}\right)\left\|\mathcal{H}_{\alpha} u\right\|^{2}+\left\|A^{\alpha} u\right\|^{2} \\
& \geq 2\left(1-\tan ^{2} \frac{\pi \alpha}{2}\right)^{\frac{1}{2}}\left\|\mathcal{H}_{\alpha} u\right\|\left\|A^{\alpha} u\right\| \\
& \geq 2 \frac{\left(\cos ^{2} \frac{\pi \alpha}{2}-\operatorname{sen}^{2} \frac{\pi \alpha}{2}\right)^{\frac{1}{2}}}{\cos \frac{\pi \alpha}{2}}\left\|\mathcal{H}_{\alpha} u\right\|\left\|A^{\alpha} u\right\| \\
& =2 \frac{(\cos \pi \alpha)^{\frac{1}{2}}}{\cos \frac{\pi \alpha}{2}}\left\|\mathcal{H}_{\alpha} u\right\|\left\|A^{\alpha} u\right\|
\end{aligned}
$$

e (5) segue.

Passo2. Em seguida assuma que $A$ é ilimitado mas ainda tem inversa limitada. Seja

$$
J_{n}=\left(I+n^{-1} A\right)^{-1}, \quad A_{n}=A J_{n}=n\left(I-J_{n}\right), n=1,2,3, \cdots
$$

Observe que $I=\left(I+n^{-1} A\right)\left(I+n^{-1} A\right)^{-1}$. Então

$$
\left\|J_{n}\right\|=\left\|\left(I+n^{-1} A\right)^{-1}\right\|=\left\|\left(n^{-1}(n+A)\right)^{-1}\right\|=\left\|n(n+A)^{-1}\right\| \leq 1
$$

para todo $n$ pois $A$ é do tipo $(\pi / 2,1)$. Portanto os $A_{n}$ são também limitados, ou seja,

$$
\left\|A_{n} x\right\| \leq\left\|n\left(I-J_{n}\right)\right\|\|x\| \leq n\left(\|I\|+\left\|J_{n}\right\|\right)\|x\| \leq 2 n\|x\|
$$


e de

$$
\begin{aligned}
\left\langle A_{n} u, u\right\rangle & =\left\langle A J_{n} u,\left(I+n^{-1} A\right) J_{n} u\right\rangle=\left\langle A J_{n} u, J_{n} u\right\rangle+n^{-1}\left\|A J_{n} u\right\|^{2} \\
& =\left\langle A J_{n} u, J_{n} u\right\rangle+n^{-1}\left\|A_{n} u\right\|^{2} \geq 0
\end{aligned}
$$

conluímos que $A_{n}$ é acretivo e

$$
\left\|A_{n} u\right\|\|u\|\|u\| \geq\left\langle A_{n} u, u\right\rangle \geq n^{-1}\left\|A_{n} u\right\|^{2}
$$

assim, $\left\|A_{n} u\right\| \leq n\|u\|$, o que implica $\left\|A_{n}\right\| \leq n$. Além disso, $A_{n}^{-1}=A^{-1}+n^{-1} I$ e $A_{n}^{-1}$, $n=1,2,3, \cdots$ é uniformemente limitada, pois

$$
\left\|A_{n}^{-1}\right\|=\left\|A^{-1}+n^{-1} I\right\| \leq\left\|A^{-1}\right\|+\left\|n^{-1} I\right\| \leq M
$$

Portanto as desigualdades (1) a (5) são válidas se substituirmos $A$ por $A_{n}$ e $\mathcal{H}_{\alpha}, \mathcal{K}_{\alpha}$ respectivamente por $\mathcal{H}_{n \alpha}, \mathcal{K}_{n \alpha}$. A seguir mostraremos as mesmas desigualdades para $A$ tomando o limite quando $n \rightarrow \infty$ com as caracterizações necessárias dos domínios.

Para este fim, primeiramente note que

$$
A_{n}^{\alpha}=A^{\alpha} J_{n}^{\alpha} \supset J_{n}^{\alpha} A^{\alpha}, \quad 0 \leq \alpha \leq 1 .
$$

Aqui $J_{n}^{\alpha}=\left(I+n^{-1} A\right)^{-\alpha}$ existe pois $I+n^{-1} A$ é maximal acretivo e a relação acima segue de $J_{n}^{\alpha}=\left(A^{-1} A_{n}\right)^{\alpha}=A^{-\alpha} A_{n}^{\alpha}=A_{n}^{\alpha} A^{-\alpha}$. Note ainda que

$$
\left\|J_{n}^{\alpha}\right\| \leq 1 \quad \text { e } \quad J_{n}^{\alpha} \stackrel{n \rightarrow \infty}{\longrightarrow} I, \quad 0 \leq \alpha \leq 1
$$

A desigualdade acima segue do Lema 3.1.1. Para verificar a convergência, note que por (2.11)

$$
\left(I+n^{-1} A\right)^{-\alpha}=\frac{\operatorname{sen} \pi \alpha}{\pi} \int_{0}^{\infty} \frac{\left(\mu+I+n^{-1} A\right)^{-1}}{\mu^{\alpha}} d \mu .
$$

E como

$$
n(\mu+1)(n(\mu+1)+A)^{-1} \frac{1}{\mu+1} e \stackrel{n \rightarrow \infty}{\longrightarrow} \frac{1}{\mu+1} e
$$

$\mathrm{e}$

$$
\left\|\frac{(\mu+1+A)^{-1}}{\mu^{\alpha}}\right\| \leq \frac{1}{(\mu+1) \mu^{\alpha}},
$$

segue do Teorema da Convergência Dominada de Lebesgue que

$$
J_{n}^{\alpha}=\left(I+n^{-1} A\right)^{-\alpha} e \longrightarrow \frac{\operatorname{sen} \pi \alpha}{\pi} \int_{0}^{\infty} \frac{1}{(\mu+1) \mu^{\alpha}} d \mu e=e .
$$

Suponha agora que $u \in D\left(A^{\alpha}\right)$ então $A_{n}^{\alpha} u=J_{n}^{\alpha} A^{\alpha} u$ e portanto

$$
\left\|A_{n}^{\alpha} u\right\| \leq\left\|A^{\alpha} u\right\|, \quad n \geq 1
$$




$$
A_{n}^{\alpha} u \stackrel{n \rightarrow \infty}{\longrightarrow} A^{\alpha} u
$$

mas para $0 \leq \alpha \leq \frac{1}{2}$

$$
\left\|A_{n}^{* \alpha} u\right\| \leq \tan \frac{\pi(1+2 \alpha)}{4}\left\|A_{n}^{\alpha}\right\| \leq \tan \frac{\pi(1+2 \alpha)}{4}\left\|A^{\alpha} u\right\|
$$

pois (3) vale para $A_{n}$. Isto mostra que $\left\|A_{n}^{* \alpha} u\right\|$ é limitada e portanto toda subseqüência possui subseqüência fracamente convergente. Ainda, para $v \in D\left(A^{\alpha}\right)$,

$$
\left\langle A_{n}^{* \alpha} u, v\right\rangle=\left\langle A_{n}^{\alpha *} u, v\right\rangle=\left\langle u, A_{n}^{\alpha} v\right\rangle \stackrel{n \rightarrow \infty}{\longrightarrow}\left\langle u, A^{\alpha} v\right\rangle
$$

Assim, $A_{n}^{* \alpha} u$ é fracamente convergente. Seja $f$ o seu limite. Então, a expressão acima nos diz que $\langle f, v\rangle=\left\langle u, A^{\alpha} v\right\rangle$, para todo $v \in D\left(A^{\alpha}\right)$. Isto implica que $u \in D\left(A^{* \alpha}\right)=D\left(A^{\alpha *}\right)$ e $f=$ $A^{* \alpha} u=A^{\alpha *} u$. O mesmo argumento mostra que $A_{n}^{* \alpha} u \stackrel{s}{\longrightarrow} A^{* \alpha} u$. Em vista da relação simétrica entre $A$ e $A^{*}$ fica provado que $D\left(A^{\alpha}\right)=D\left(A^{* \alpha}\right)=D_{\alpha}$ e que $A_{n}^{\alpha} u \rightarrow A^{\alpha} u, A_{n}^{* \alpha} u \rightarrow A^{* \alpha} u$, para todo $u \in D_{\alpha}$. Os operadores $\mathcal{H}_{\alpha}$ e $\mathcal{K}_{\alpha}$ definidos anteriormente tem domínio $D_{\alpha}$ e $\mathcal{H}_{n \alpha} u \rightarrow \mathcal{H}_{\alpha} u$, $\mathcal{K}_{n \alpha} u \rightarrow \mathcal{K}_{\alpha} u$, para todo $u \in D_{\alpha}$. Segue das desigualdades (1) a (5) para $A_{n}^{\alpha}, A_{n}^{* \alpha}, \mathcal{H}_{n \alpha}$ e $\mathcal{K}_{n \alpha}$, $u \in D_{\alpha}$, tomando o limite quando $n \rightarrow \infty$, que as desigualdades (1) a (5) para $A^{\alpha}, A^{* \alpha}, \mathcal{H}_{\alpha} \mathrm{e}$ $\mathcal{K}_{\alpha}$ valem para $u \in D_{\alpha}$.

Observação 3.1.1 O teorema acima é devido a Kato que em [16] prova uma versão mais geral do resultado acima, sem a hipótese de $0 \in \rho(A)$.

Teorema 3.1.2 Seja A um operador limitado e maximal acretivo em um espaço de Hilbert $H$. Então $A^{\alpha}$ pode ser estendido a a complexo de forma que seja analítico para $\operatorname{Re} \alpha>0$ e

$$
\left\|A^{\alpha}\right\| \leq \frac{\operatorname{sen} \pi \xi^{\prime}}{\pi \xi^{\prime}\left(1-\xi^{\prime}\right)}\|A\|^{\xi} \mathbf{e}^{\pi \frac{|\eta|}{2}} \leq \frac{4}{\pi}\|A\|^{\xi} \mathbf{e}^{\pi \frac{|\eta|}{2}}, \quad \alpha=\xi+i \eta, \quad \xi^{\prime}=\xi-[\xi]
$$

Se A não tem autovalor nulo $A^{\alpha}$ pode ser estendido a $\operatorname{Re} \alpha \geq 0$ de forma que $A^{\alpha}$ é fortemente contínuo e (3.10) vale para $\operatorname{Re} \alpha \geq 0$. Em particular $A^{\text {in }}$ é um semigrupo fortemente contínuo em $\eta \operatorname{com}\left\|A^{i \eta}\right\| \leq \mathbf{e}^{\pi \frac{|\eta|}{2}}$.

Prova. As potências $A^{\alpha}$ de $A$ podem ser definidas para $0<\operatorname{Re} \alpha<1$ por

$$
A^{\alpha}=\frac{\operatorname{sen} \pi \alpha}{\pi} \int_{0}^{\infty} \lambda^{\alpha-1} A(\lambda+A)^{-1} d \lambda .
$$


Já vimos que $A^{\alpha}$ é analítica para $\operatorname{Re} \alpha>0$ e que $A^{\alpha} A^{\beta}=A^{\alpha+\beta}$ para $\alpha$ e $\beta$ com parte real positiva. Segue que, para $0<\xi<1$

$$
\begin{aligned}
\left\|A^{\xi}\right\| & \leq \frac{\operatorname{sen} \pi \xi}{\pi}\left\{\int_{0}^{\infty}\left\|\lambda^{\xi-1} A(\lambda+A)^{-1}\right\| d \lambda\right\} \\
& \leq \frac{\operatorname{sen} \pi \xi}{\pi}\left\{\int_{0}^{\|A\|} \lambda^{\xi-1} d \lambda+\|A\| \int_{\|A\|}^{\infty} \lambda^{\xi-2} d \lambda\right\} \\
& \leq \frac{\operatorname{sen} \pi \xi}{\pi}\left\{\frac{\|A\|^{\xi}}{\xi}+\frac{\|A\|^{\xi}}{1-\xi}\right\} \\
& =\frac{\operatorname{sen} \pi \xi}{\pi} \frac{\|A\|^{\xi}}{\xi(1-\xi)} \leq \frac{4}{\pi}\|A\|^{\xi}
\end{aligned}
$$

onde usamos que $\left\|A(\lambda+A)^{-1}\right\| \leq \min \left(1, \lambda^{-1}\|A\|\right)$. Assuma por um instante que $\operatorname{Re} A \geq \delta>0$ de forma que $A^{\alpha}$ está definido para todo $\alpha$ complexo e mostremos que

$$
\left\|A^{i \eta}\right\| \leq \mathbf{e}^{\pi \frac{|\eta|}{2}}
$$

Disto segue (3.10) notando que $A^{\alpha}=A^{\xi+i \eta}=A^{[\xi]} A^{\xi^{\prime}} A^{i \eta}$. De fato,

$$
\left\|A^{\alpha}\right\|=\left\|A^{[\xi]}\right\|\left\|A^{\xi^{\prime}}\right\|\left\|A^{i \eta}\right\| \leq\|A\|^{\xi-\xi^{\prime}} \frac{4}{\pi}\|A\|^{\xi^{\prime}} \mathbf{e}^{\pi \frac{|\eta|}{2}}=\frac{4}{\pi}\|A\|^{\xi} \mathbf{e}^{\pi \frac{|\eta|}{2}} .
$$

O caso geral segue substituindo-se $A$ por $A+\epsilon$ e fazendo $\epsilon \rightarrow 0$.

Para mostrar (3.11) observe que $A^{\alpha}=\mathcal{H}_{\alpha}+i \mathcal{K}_{\alpha}$ e $A^{* \alpha}=\mathcal{H}_{\alpha}-i \mathcal{K}_{\alpha},\left\|\mathcal{K}_{\alpha} \mathcal{H}_{\alpha}^{-1}\right\| \leq\left|\tan \frac{\pi \alpha}{2}\right|$. Portanto

$$
\begin{aligned}
\left\|A^{* \alpha} A^{-\alpha}\right\| & =\frac{\left\|\mathcal{H}_{\alpha}-i \mathcal{K}_{\alpha}\right\|}{\mathcal{H}_{\alpha}+i \mathcal{K}_{\alpha}}=\frac{\left\|\mathcal{H}_{\alpha}\right\|\left\|1-i \mathcal{K}_{\alpha} \mathcal{H}_{\alpha}^{-1}\right\|}{\left\|\mathcal{H}_{\alpha}\right\|\left\|1-\left(-i \mathcal{K}_{\alpha} \mathcal{H}_{\alpha}^{-1}\right)\right\|} \\
& \leq \frac{1+\left|\tan \frac{\pi \alpha}{2}\right|}{\left\|1-\left(-i \mathcal{K}_{\alpha} \mathcal{H}_{\alpha}^{-1}\right)\right\|} \leq \frac{1+\left|\tan \frac{\pi \alpha}{2}\right|}{\left\|1\left|-\left\|\mathcal{K}_{\alpha} \mathcal{H}_{\alpha}^{-1}\right\|\right|\right.} \\
& =\frac{1+\left|\tan \frac{\pi \alpha}{2}\right|}{1-\left\|\mathcal{K}_{\alpha} \mathcal{H}_{\alpha}^{-1}\right\|} \leq \frac{1+\left|\tan \frac{\pi \alpha}{2}\right|}{1-\left|\tan \frac{\pi \alpha}{2}\right|}
\end{aligned}
$$

que para $\alpha=i \eta$ nos dá

$$
\left\|A^{i \eta}\right\|^{2} \leq\left\|A^{*-i \eta} A^{i \eta}\right\| \leq \frac{1+\tan \frac{\pi \alpha}{2}}{1-\left|\tan \frac{\pi \alpha}{2}\right|}=\mathbf{e}^{\pi|\eta|}
$$

provando (3.11). Aqui usamos que

$$
\left\langle A^{*-i \eta} A^{i \eta} u, u\right\rangle=\left\langle A^{i \eta} u, A^{*-i \eta *} u\right\rangle=\left\|A^{i \eta} u\right\|^{2}
$$

para concluir a primeira igualdade em (3.12) e

$$
\tan \pi \frac{i \eta}{2}=\frac{\mathbf{e}^{-\pi \frac{\eta}{2}}-\mathbf{e}^{\pi \frac{\eta}{2}}}{\mathbf{e}^{-\pi \frac{\eta}{2}}+\mathbf{e}^{\pi \frac{\eta}{2}}} i
$$


então temos

$$
\left|\tan \pi \frac{i \eta}{2}\right|=\frac{\left|\mathbf{e}^{-\pi \frac{\eta}{2}}-\mathbf{e}^{\pi \frac{\eta}{2}}\right|}{\left|\mathbf{e}^{-\pi \frac{\eta}{2}}+\mathbf{e}^{\pi \frac{\eta}{2}}\right|}=\frac{\left|2 \mathbf{e}^{-\pi \frac{\eta}{2}}-\mathbf{e}^{\pi \frac{\eta}{2}}-\mathbf{e}^{-\pi \frac{\eta}{2}}\right|}{\mathbf{e}^{-\pi \frac{\eta}{2}}+\mathbf{e}^{\pi \frac{\eta}{2}}}=\frac{2 \mathbf{e}^{-\pi \frac{\eta}{2}}}{\mathbf{e}^{-\pi \frac{\eta}{2}}+\mathbf{e}^{\pi \frac{\eta}{2}}}-1
$$

e

$$
-\left|\tan \pi \frac{i \eta}{2}\right|=-\frac{\left|\mathbf{e}^{-\pi \frac{\eta}{2}}-\mathbf{e}^{\pi \frac{\eta}{2}}\right|}{\left|\mathbf{e}^{-\pi \frac{\eta}{2}}+\mathbf{e}^{\pi \frac{\eta}{2}}\right|}=-\frac{\left|-2 \mathbf{e}^{\pi \frac{\eta}{2}}+\mathbf{e}^{-\pi \frac{\eta}{2}}+\mathbf{e}^{\pi \frac{\eta}{2}}\right|}{\mathbf{e}^{-\pi \frac{\eta}{2}}+\mathbf{e}^{\pi \frac{\eta}{2}}}=\frac{2 \mathbf{e}^{\pi \frac{\eta}{2}}}{\mathbf{e}^{-\pi \frac{\eta}{2}}+\mathbf{e}^{\pi \frac{\eta}{2}}}-1
$$

assim, obtemos que

$$
\frac{1+\left|\tan \pi \frac{i \eta}{2}\right|}{1-\left|\tan \pi \frac{i \eta}{2}\right|}=\mathbf{e}^{\pi|\eta|}
$$

Mostremos que $\alpha \mapsto A^{\alpha}$ é contínuo para $u \in H, \alpha \in\{\xi+i \eta \in \mathbb{C}: 0<\xi \leq 1,|\eta| \leq R\}=D$. Como $A^{\alpha}$ é limitado para $\alpha \in D$ por (3.10) é suficiente mostra isto para um denso de $H$. Se $A$ não tem autovalor nulo a imagem de $A$ é densa como mostra o Lema a seguir, logo é suficiente mostrar que isto vale para $u=A v$. Então $A^{\alpha} u=A^{1+\alpha} v$ e isto é obviamente uniformemente contínuo em $D$.

Lema 3.1.7 Seja A fechado e maximal acretivo em um espaço de Hilbert $H$, então

$$
H=\overline{D(A)}
$$

Se A é fechado e maximal acretivo e 0 não é um auto-valor de $A$ então $\overline{R(A)}=H$.

Prova. Como $A$ é fechado, maximal acretivo e $H$ é Hilbert, segue que $A$ é do tipo $(\pi / 2,1), \operatorname{logo}$ $A$ tem domínio denso. A segunda afirmativa segue do fato que se $A$ é fechado, maximal acretivo e 0 não é um autovalor de $A$ então sua inversa sobre a imagem é um operador fechado e maximal acretivo. 


\section{Capítulo 4}

\section{Métodos de Interpolação Complexos}

Neste capítulo vamos apresentar a teoria dos espaços de interpolação complexa. Iniciaremos com algumas definições necessárias para o desenvolvimento deste Capítulo, e em seguida apresentaremos alguns resultados que podem ser encontrados em $[6,8]$.

\subsection{Introdução}

Sejam $A_{0}$ e $A_{1}$ espaços de Banach e $\mathcal{A}$ um espaço vetorial topológico de Hausdorff tal que $A_{i} \subset \mathcal{A}, i=0,1$. Neste caso dizemos que $\left\{A_{0}, A_{1}\right\}$ é um par de interpolação.

Agora, considere os espaços $A_{0} \cap A_{1}$, com a norma

$$
\|a\|_{A_{0} \cap A_{1}}=\max \left(\|a\|_{A_{0}},\|a\|_{A_{1}}\right)
$$

e

$$
\begin{gathered}
A_{0}+A_{1}=\left\{a: a \in \mathcal{A}, a=a_{0}+a_{1}, a_{j} \in A_{j}, j=0,1\right\} \\
\|a\|_{A_{0}+A_{1}}=\inf _{\substack{a=a_{0}+a_{1} \\
a_{j} \in A_{j}}}\left\{\left\|a_{0}\right\|_{A_{0}}+\left\|a_{1}\right\|_{A_{1}}\right\}
\end{gathered}
$$

O ínfimo é tomado sobre todos os representantes de $a \in A_{0}+A_{1}$ no sentido descrito.

Lema 4.1.1 Seja $\left\{A_{0}, A_{1}\right\}$ um par de interpolação. Então $A_{0} \cap A_{1}$ e $A_{0}+A_{1}$ são espaços de Banach. Ainda

$$
A_{0} \cap A_{1} \hookrightarrow A_{j} \hookrightarrow A_{0}+A_{1}, \quad j=0,1
$$

onde $\hookrightarrow$ significa inclusão contínua.

Prova. Primeiramente, mostramos que $A_{0} \cap A_{1}$ é um espaço normado. De fato,

$$
\|a\|_{A_{0} \cap A_{1}}=0 \Longrightarrow \max \left(\|a\|_{A_{0}},\|a\|_{A_{1}}\right)=0 \Longrightarrow a=0
$$


(ii)

$$
\|\lambda a\|_{A_{0} \cap A_{1}}=\max \left(\|\lambda a\|_{A_{0}},\|\lambda a\|_{A_{1}}\right)=|\lambda| \max \left(\|a\|_{A_{0}},\|a\|_{A_{1}}\right) .
$$

(iii) Se $a, b \in A_{0} \cap A_{1}$, então $a+b \in A_{0} \cap A_{1}$, e

$$
\begin{aligned}
\|a+b\|_{A_{0} \cap A_{1}} & =\max \left(\|a+b\|_{A_{0}},\|a+b\|_{A_{1}}\right) \\
& \leq \max \left(\|a\|_{A_{0}},\|a\|_{A_{1}}\right)+\max \left(\|b\|_{A_{0}},\|b\|_{A_{1}}\right) \\
& =\|a\|_{A_{0} \cap A_{1}}+\|b\|_{A_{0} \cap A_{1}} .
\end{aligned}
$$

Agora tome $\left\{x_{n}\right\} \subset A_{0} \cap A_{1}$ uma seqüência de Cauchy, então $x_{n}$ é uma seqüência de Cauchy em $A_{0}$ e portanto $x_{n} \longrightarrow x \in A_{0}, \operatorname{logo} x_{n} \longrightarrow x$ em $\mathcal{A}$, ainda, $x_{n}$ é também uma seqüência de Cauchy em $A_{1}$ e assim $x_{n} \longrightarrow y \in A_{1}, \operatorname{logo} x_{n} \longrightarrow y$ em $\mathcal{A}$, como o limite é único em $\mathcal{A}$, $x=y \in A_{0} \cap A_{1}$. Agora mostremos que $\|a\|_{A_{0}+A_{1}}$ é uma norma em $A_{0}+A_{1}$.

(i) $\|a\|_{A_{0}+A_{1}}=0$, temos que existem seqüências $\left\{a_{j}^{n}\right\} \in A_{j}, j=0,1$ tais que

$$
\left\|a_{0}^{n}\right\|_{A_{0}}+\left\|a_{1}^{n}\right\|_{A_{1}} \stackrel{n \rightarrow \infty}{\longrightarrow} 0, \quad a=a_{0}^{n}+a_{1}^{n}
$$

Logo, $a_{j}^{n} \longrightarrow 0$ em $A_{j}, j=0,1$. Assim $a=a_{0}^{n}+a_{1}^{n} \stackrel{\mathcal{A}}{\longrightarrow} 0$ e $a=0$.

(ii) $\lambda a=a_{0}^{\lambda}+a_{1}^{\lambda}$ então se $\lambda \neq 0$, temos

$$
\begin{aligned}
\|\lambda a\|_{A_{0}+A_{1}} & =\inf _{\substack{a_{j}^{\lambda} \in A_{j} \\
a_{0}^{\lambda}+a_{1}^{\lambda}=\lambda a}}\left\{\left\|a_{0}^{\lambda}\right\|_{A_{0}}+\left\|a_{1}^{\lambda}\right\|_{A_{1}}\right\} \\
= & \inf _{\substack{a_{j}^{\lambda} \\
\frac{a_{j}^{\lambda}}{\lambda} \in A_{j} \\
\frac{a_{0}^{\lambda}}{\lambda}+\frac{a_{1}^{\lambda}}{\lambda}=a}}\left\{\left\|a_{0}^{\lambda}\right\|_{A_{0}}+\left\|a_{1}^{\lambda}\right\|_{A_{1}}\right\} \\
= & \inf _{\substack{a_{j} \in A_{j} \\
a_{0}+a_{1}=a}}\left\{\left\|\lambda a_{0}\right\|_{A_{0}}+\left\|\lambda a_{1}\right\|_{A_{1}}\right\} \\
= & |\lambda| \inf _{\substack{a_{j} \in A_{j} \\
a_{0}+a_{1}=a}}\left\{\left\|a_{0}\right\|_{A_{0}}+\left\|a_{1}\right\|_{A_{1}}\right\}=|\lambda|\|a\|_{A_{0}+A_{1}}
\end{aligned}
$$

(iii) Se $a \in A_{0}+A_{1}$, então $a=a_{0}+a_{1}, a_{j} \in A_{j}$ e $b \in A_{0}+A_{1}$, então $b=b_{0}+b_{1}, b_{j} \in A_{j}$, $\operatorname{logo}$ se $a+b=\left(a_{0}+b_{0}\right)+\left(a_{1}+b_{1}\right)$, existe $\left\{a_{j}^{n}\right\}$ e $\left\{b_{j}^{n}\right\}$ em $A_{j}$ tal que $a=a_{0}^{n}+a_{1}^{n}$ e $b=b_{0}^{n}+b_{1}^{n}$, $\left\|a_{0}^{n}\right\|_{A_{0}}+\left\|a_{1}^{n}\right\|_{A_{1}} \longrightarrow\|a\|_{A_{0}+A_{1}}$ e $\left\|b_{0}^{n}\right\|_{A_{0}}+\left\|b_{1}^{n}\right\|_{A_{1}} \longrightarrow\|b\|_{A_{0}+A_{1}}$, segue que

$$
\begin{aligned}
\|a+b\|_{A_{0}+A_{1}} & =\inf _{\substack{a+b=c_{0}+c_{1} \\
c_{j} \in A_{j}}}\left\{\left\|c_{0}\right\|_{A_{0}}+\left\|c_{1}\right\|_{A_{1}}\right\} \\
& \leq\left\|a_{0}^{n}\right\|_{A_{0}}+\left\|a_{1}^{n}\right\|_{A_{1}}+\left\|b_{0}^{n}\right\|_{A_{0}}+\left\|b_{1}^{n}\right\|_{A_{1}}
\end{aligned}
$$

fazendo $n \rightarrow \infty$, obtemos que $\|a+b\|_{A_{0}+A_{1}} \leq\|a\|_{A_{0}+A_{1}}+\|b\|_{A_{0}+A_{1}}$. 
Mostremos agora, que as inclusões abaixo são contínuas,

$$
\begin{aligned}
i: A_{0} \cap A_{1} & \longrightarrow A_{0} \\
a & \longmapsto a
\end{aligned}
$$

Observe que

$$
\|i(a)\|_{A_{0}}=\|a\|_{A_{0}} \leq \max \left\{\|a\|_{A_{0}},\|a\|_{A_{1}}\right\} \leq\|a\|_{A_{0} \cap A_{1}} .
$$

Portanto a inclusão é contínua. Analogamente mostra-se que

$$
\begin{aligned}
i: A_{0} \cap A_{1} & \longrightarrow A_{1} \\
a & \longmapsto a
\end{aligned}
$$

é contínua. Ainda

$$
\begin{aligned}
i: A_{1} & \longrightarrow A_{0}+A_{1} \\
a & \longmapsto a
\end{aligned}
$$

temos

$$
\|i(a)\|_{A_{0}+A_{1}}=\|a\|_{A_{0}+A_{1}}=\|0+a\|_{A_{0}+A_{1}} \leq\|0\|_{A_{0}}+\|a\|_{A_{1}} \leq\|a\|_{A_{1}} .
$$

O que mostra que a inclusão acima é contínua, analogamente mostra-se que

$$
\begin{aligned}
i: A_{0} & \longrightarrow A_{0}+A_{1} \\
a & \longmapsto a
\end{aligned}
$$

é contínua.

Resta mostrar que $A_{0}+A_{1}$ é completo, para isto seja $\left\{a^{n}\right\}$ uma seqüência de Cauchy em $A_{0}+A_{1}$. Então existem números $1<n_{1}<n_{2}<\ldots$ tais que

$$
a^{n_{j+1}}-a^{n_{j}}=b_{0}^{j}+b_{1}^{j}, \quad b_{i}^{j} \in A_{i}, i=0,1
$$

$\mathrm{e}$

$$
\sum_{j=1}^{\infty}\left\|b_{0}^{j}\right\|_{A_{0}}+\left\|b_{1}^{j}\right\|_{A_{1}}<\infty
$$

Para um elemento conveniente $a \in A_{0}+A_{1}$, obtemos $b_{i} \in A_{i}$, tais que $\sum_{j=1}^{N-1} b_{i}^{j} \stackrel{A_{i}}{\longrightarrow} b_{i}$, com

$$
a^{n_{N}}-a^{n_{1}}=\sum_{k=1}^{N-1}\left(b_{0}^{k}+b_{1}^{k}\right) \stackrel{A_{0}+A_{1}}{\longrightarrow} b_{0}+b_{1}=a-a^{n_{1}} \in A_{0}+A_{1} .
$$

Assim, $a^{n_{N}}$ (e logo a seqüência $\left\{a_{n}\right\}$ também) converge em $A_{0}+A_{1}$ para $a$.

\subsection{Funtores de Interpolação}

Seja $\mathcal{B}$ o conjunto de todos os espaços de Banach. Para cada par de espaços $(A, B)$ os morfismos de $A$ em $B$ são as transformações lineares e contínuas de $A$ em $B, L(A, B)$. Para cada 
$T \in L(A, B)$ e $S \in L(B, C)$ definimos $S \circ T \in L(A, C)$, a composição de $S \operatorname{com} T$. A composição é associativa $(T \circ S) \circ R=T \circ(S \circ R)$ e existe $I_{A} \in L(A, A)$ tal que

$$
G \circ I_{A}=G, \quad \forall G \in L(A, B)
$$

com estas propriedades $\mathcal{B}$ é uma categoria.

Seja $\mathcal{C}=\left\{\left\{A_{0}, A_{1}\right\}:\left\{A_{0}, A_{1}\right\}\right.$ é um par de interpolação $\}$ e defina os morfismos entre dois elementos de $\mathcal{C},\left\{A_{0}, A_{1}\right\}$ e $\left\{B_{0}, B_{1}\right\}$ por

$$
\begin{aligned}
& T: A_{0}+A_{1} \longrightarrow B_{0}+B_{1} \\
& \left.T\right|_{A_{i}}: \quad A_{i} \quad \longrightarrow B_{i}, i=0,1 \text {, está em } L\left(A_{i}, B_{i}\right)
\end{aligned}
$$

Notação: $T \in L\left(\left\{A_{0}, A_{1}\right\},\left\{B_{0}, B_{1}\right\}\right)$.

Definição 4.2.1 Um funtor de interpolação é uma função $F: \mathcal{C} \longrightarrow \mathcal{B}$ tal que

$$
\begin{gathered}
F\left(\left\{A_{0}, A_{1}\right\}\right)=A \in \mathcal{B} \\
A_{0} \cap A_{1} \hookrightarrow A \hookrightarrow A_{0}+A_{1}
\end{gathered}
$$

e dados dois pares de interpolação $\left\{A_{0}, A_{1}\right\},\left\{B_{0}, B_{1}\right\}$ e um morfismo $T \in L\left(\left\{A_{0}, A_{1}\right\},\left\{B_{0}, B_{1}\right\}\right)$, $F(T) \in L\left(F\left(\left\{A_{0}, A_{1}\right\}\right), F\left(\left\{B_{0}, B_{1}\right\}\right)\right) \operatorname{com} F(T)=\left.T\right|_{F\left(\left\{A_{0}, A_{1}\right\}\right)}$.

Um espaço de Banach que pode ser representado na forma $A=F\left(\left\{A_{0}, A_{1}\right\}\right)$ com a ajuda de um funtor apropriado de interpolação $F$ é dito ser um espaço de interpolação (com respeito a $\left.\left\{A_{0}, A_{1}\right\}\right)$.

Definição 4.2.2 Seja $f:(0, \infty)^{2} \longrightarrow(0, \infty)$ crescente e positiva com $f(1,1)=1$. Um funtor de interpolação $F$ é do tipo $f$ se existe $C>0$ tal que para todos pares de interpolção $\left\{A_{0}, A_{1}\right\}$ $e\left\{B_{0}, B_{1}\right\}$, com $A=F\left(\left\{A_{0}, A_{1}\right\}\right), B=F\left(\left\{B_{0}, B_{1}\right\}\right)$ e $T \in L\left(\left\{A_{0}, A_{1}\right\},\left\{B_{0}, B_{1}\right\}\right)$ temos a desigualdade

$$
\|T\|_{L(A, B)} \leq C f\left(\|T\|_{L\left(A_{0}, B_{0}\right)},\|T\|_{L\left(A_{1}, B_{1}\right)}\right) .
$$

Ainda, F é exato se $C=1$ e de expoente $\theta$ se $f\left(t_{0}, t_{1}\right)=t_{0}^{1-\theta} t_{1}^{\theta}, 0 \leq \theta \leq 1$.

Observação 4.2.1 Se F é um funtor de interpolação do tipo $f$. Escolhendo $A_{0}=B_{0}, A_{1}=B_{1}$ e $T=I_{d}$ (operador identidade), segue que

$$
1 \leq c f(1,1)=c .
$$

Lema 4.2.1 Os funtores de interpolação

$$
F\left(\left\{A_{0}, A_{1}\right\}\right)=A_{0} \cap A_{1} \text { e } F\left(\left\{A_{0}, A_{1}\right\}\right)=A_{0}+A_{1}
$$

são exatos e do tipo $f\left(t_{0}, t_{1}\right)=\max \left(t_{0}, t_{1}\right)$. 
Prova. 1) $F\left(\left\{A_{0}, A_{1}\right\}\right)=A_{0} \cap A_{1}$

$$
\begin{aligned}
\|T u\|_{B_{0} \cap B_{1}} & =\max \left\{\|T u\|_{B_{0}},\|T u\|_{B_{1}}\right\} \\
& \leq \max \left\{\|T\|_{L\left(A_{0}, B_{0}\right)}\|u\|_{A_{0}},\|T\|_{L\left(A_{1}, B_{1}\right)}\|u\|_{A_{1}}\right\} \\
& \leq \max \left\{\|T\|_{L\left(A_{0}, B_{0}\right)},\|T\|_{L\left(A_{1}, B_{1}\right)}\right\} \max \left\{\|u\|_{A_{0}},\|u\|_{A_{1}}\right\} \\
& \leq f\left(\|T\|_{L\left(A_{0}, B_{0}\right)},\|T\|_{L\left(A_{1}, B_{1}\right)}\right)\|u\|_{A_{0} \cap A_{1}} .
\end{aligned}
$$

Portanto

$$
\|T\|_{L\left(F\left(A_{0}, A_{1}\right), F\left(B_{0}, B_{1}\right)\right)} \leq f\left(\|T\|_{L\left(A_{0}, B_{0}\right)},\|T\|_{L\left(A_{1}, B_{1}\right)}\right) .
$$

2) $F\left(\left\{A_{0}, A_{1}\right\}\right)=A_{0}+A_{1}$

$$
\begin{aligned}
\|T u\|_{B_{0}+B_{1}} & \leq \inf _{\substack{u=u_{0}+u_{1} \\
u_{i} \in A_{i}, i=0,1}}\left\{\left\|T u_{0}\right\|_{B_{0}}+\left\|T u_{1}\right\|_{B_{1}}\right\} \\
& \leq \inf _{\substack{u=u_{0}+u_{1} \\
u_{i} \in A_{i}, i=0,1}}\left\{\|T\|_{L\left(A_{0}, B_{0}\right)}\left\|u_{0}\right\|_{A_{0}}+\|T\|_{L\left(A_{1}, B_{1}\right)}\left\|u_{1}\right\|_{A_{1}}\right\} \\
& \leq \max \left\{\|T\|_{L\left(A_{0}, B_{0}\right)},\|T\|_{L\left(A_{1}, B_{1}\right)}\right\} \inf _{\substack{u=u_{0}+u_{1} \\
u_{i} \in A_{i}, i=0,1}}\left\{\left\|u_{0}\right\|_{A_{0}}+\left\|u_{1}\right\|_{A_{1}}\right\} \\
& =f\left(\|T\|_{L\left(A_{0}, B_{0}\right)},\|T\|_{L\left(A_{1}, B_{1}\right)}\right)\|u\|_{A_{0}+A_{1} .} .
\end{aligned}
$$

Portanto

$$
\|T\|_{L\left(F\left(A_{0}, A_{1}\right), F\left(B_{0}, B_{1}\right)\right)} \leq f\left(\|T\|_{L\left(A_{0}, B_{0}\right)},\|T\|_{L\left(A_{1}, B_{1}\right)}\right) .
$$

\subsection{Métodos Complexos de Interpolação}

Vamos denotar por $S$ a faixa

$$
S=\{z: 0<\operatorname{Re} z<1\}
$$

do plano complexo e $\bar{S}$ o seu fecho.

Definição 4.3.1 Seja $\left\{A_{0}, A_{1}\right\}$ um par de interpolação. Seja $\gamma$ um número real. Então por 
definição

$$
\begin{aligned}
F\left(A_{0}, A_{1}, \gamma\right)= & \left\{f: f(z) \text { é }\left(A_{0}+A_{1}\right) \text {-contínua em } \bar{S} e\right. \\
& \left(A_{0}+A_{1}\right) \text {-analítica em } S, \\
& \sup _{z \in \bar{S}} \mathbf{e}^{-|\gamma||\operatorname{Im} z|}\|f(z)\|_{A_{0}+A_{1}}<\infty, \\
& f(j+i t) \in A_{j}, j=0,1,-\infty<t<\infty, \\
& f(j+i t) e ́ A_{j} \text {-contínua com respeito at, } j=0,1, \\
& \left.\|f\|_{F(\gamma)}=\max _{j=0,1}\left(\sup _{t} \mathbf{e}^{-\gamma|t|}\|f(j+i t)\|_{A_{j}}\right)<\infty\right\} .
\end{aligned}
$$

$F_{-}\left(A_{0}, A_{1}, \gamma\right)$ é o conjunto de todas funções $f \in F\left(A_{0}, A_{1}, \gamma\right)$ com

$$
\lim _{|t| \rightarrow \infty} \mathbf{e}^{-\gamma|t|}\|f(j+i t)\|_{A_{j}}=0, \quad j=0,1 .
$$

Teorema 4.3.1 (Teorema das Três Linhas) Seja $\varphi$ uma função contínua e limitada na faixa $0 \leq \operatorname{Re} z \leq 1$ que é analítica no interior da faixa. Se $|\varphi(z)| \leq M_{0}$ para $\operatorname{Re} z=0$ e $|\varphi(z)| \leq M_{1}$ para $\operatorname{Re} z=1$, então $|\varphi(z)| \leq M_{0}^{1-t} M_{1}^{t}$ para $\operatorname{Re} z=t, 0<t<1$.

Prova. Ver [12].

Teorema 4.3.2 (a) $F\left(A_{0}, A_{1}, \gamma\right)$ e $F_{-}\left(A_{0}, A_{1}, \gamma\right)$ equipados com a norma (4.2) são espaços de Banach.

(b) O gerado pelas funções $\mathbf{e}^{\delta z^{2}+\lambda z} a, \delta>0, \lambda$ real; $a \in A_{0} \cap A_{1}$, é denso em $F_{-}\left(A_{0}, A_{1}, \gamma\right)$.

Prova. Passo1. Mostremos que $\|f\|_{F(\gamma)}$ definida em (4.2) é uma norma.

1) Se $\|f(z)\|_{F(\gamma)}=0$, então $f(z)=0$. De fato, se $f \in F\left(A_{0}, A_{1}, \gamma\right), z_{0} \in S$ e $\delta>0$, considere a aplicação $h(z)=\mathbf{e}^{\delta\left(z-z_{0}\right)^{2}} f(z), z=\nu+i t$, então defina

$$
M(\nu)=\sup _{t}\left\|\mathbf{e}^{\delta\left(\nu+i t-z_{0}\right)^{2}} f(\nu+i t)\right\|_{A_{0}+A_{1}}
$$

Do Teorema das Três Linhas temos que

$$
M(\nu) \leq M(0)^{1-\nu} M(1)^{\nu}
$$

assim,

$$
M(\nu) \leq\left(\sup _{t}\left\|\mathbf{e}^{\delta\left(1+i t-z_{0}\right)^{2}} f(1+i t)\right\|_{A_{0}+A_{1}}\right)^{\nu}\left(\sup _{t}\left\|\mathbf{e}^{\delta\left(i t-z_{0}\right)^{2}} f(i t)\right\|_{A_{0}+A_{1}}\right)^{1-\nu}
$$

Então,

$$
\begin{aligned}
& \left\|f\left(z_{0}\right)\right\|_{A_{0}+A_{1}} \\
& \leq\left(\sup _{t}\left\|\mathbf{e}^{\delta\left(1+i t-z_{0}\right)^{2}} f(1+i t)\right\|_{A_{0}+A_{1}}\right)^{\operatorname{Re} z_{0}}\left(\sup _{t}\left\|\mathbf{e}^{\delta\left(i t-z_{0}\right)^{2}} f(i t)\right\|_{A_{0}+A_{1}}\right)^{1-\operatorname{Re} z_{0}}
\end{aligned}
$$

Se $\|f(z)\|_{F(\gamma)}=0$ então a última estimativa mostra que $f(z)=0$. 
2) Se $f \in F\left(A_{0}, A_{1}, \gamma\right)$ e $-\infty<\lambda<\infty$,

$$
\begin{aligned}
\|\lambda f\|_{F(\gamma)} & =\max _{j=0,1}\left(\sup _{t} \mathbf{e}^{-\gamma|t|}\|\lambda f(j+i t)\|_{A_{j}}\right) \\
& =\max _{j=0,1}\left(\sup _{t}|\lambda| \mathbf{e}^{-\gamma|t|}\|f(j+i t)\|_{A_{j}}\right) \\
& =|\lambda| \max _{j=0,1}\left(\sup _{t} \mathbf{e}^{-\gamma|t|}\|f(j+i t)\|_{A_{j}}\right) \\
& =|\lambda|\|f\|_{F(\gamma)}
\end{aligned}
$$

3) Se $f, g \in F\left(A_{0}, A_{1}, \gamma\right)$,

$$
\begin{aligned}
\|f+g\|_{F(\gamma)} & =\max _{j=0,1}\left(\sup _{t} \mathbf{e}^{-\gamma|t|}\|f(j+i t)+g(j+i t)\|_{A_{j}}\right) \\
& \leq \max _{j=0,1}\left(\sup _{t} \mathbf{e}^{-\gamma|t|}\left(\|f(j+i t)\|_{A_{j}}+\|g(j+i t)\|_{A_{j}}\right)\right) \\
& \leq \max _{j=0,1}\left(\sup _{t} \mathbf{e}^{-\gamma|t|}\|f(j+i t)\|_{A_{j}}+\sup _{t} \mathbf{e}^{-\gamma|t|}\|g(j+i t)\|_{A_{j}}\right) \\
& \leq\|f\|_{F(\gamma)}+\|g\|_{F(\gamma)} .
\end{aligned}
$$

Para mostrar que $F\left(A_{0}, A_{1}, \gamma\right)$ é completo, iniciemos com uma seqüência de Cauchy $\left\{f_{k}(z)\right\}_{k=1}^{\infty} \subset$ $F\left(A_{0}, A_{1}, \gamma\right)$. Mostremos que $\left\{f_{k}(j+i t)\right\}_{k=1}^{\infty}$ é uma seqüência de Cauchy em $A_{j}$. De fato,

$$
\begin{aligned}
\mathbf{e}^{-\gamma|t|}\left\|f_{n}(j+i t)-f_{m}(j+i t)\right\|_{A_{j}} & \leq \sup _{t} \mathbf{e}^{-\gamma|t|}\left\|\left(f_{n}-f_{m}\right)(j+i t)\right\|_{A_{j}} \\
& \leq \max _{j=0,1} \sup _{t} \mathbf{e}^{-\gamma|t|}\left\|\left(f_{n}-f_{m}\right)(j+i t)\right\|_{A_{j}} \\
& \leq\left\|f_{n}-f_{m}\right\|_{F(\gamma)} \stackrel{n, m \rightarrow \infty}{\longrightarrow} 0 .
\end{aligned}
$$

Então, $\left\{f_{k}(j+i t)\right\}_{k=1}^{\infty}$ converge em $A_{j}$ para um limite denotado por $f(j+i t)$. Assim, dado $\epsilon>0$, $\exists k_{0}(\epsilon)$ tal que

$$
\max _{j=0,1} \sup _{t} \mathbf{e}^{-\gamma|t|}\left\|f_{k}(j+i t)-f(j+i t)\right\|_{A_{j}}<\epsilon, \quad \text { se } k \geq k_{0}(\epsilon)
$$

Portanto, $f(j+i t)$ é $A_{j}$-contínua.

Substituindo $f\left(z_{0}\right)$ em (4.4) por $\mathbf{e}^{z^{2}}\left(f_{k}(z)-f_{l}(z)\right)$ e escolhendo $\delta=0$, temos que

$$
\left\|\mathbf{e}^{z^{2}}\left(f_{k}-f_{l}\right)\right\|_{A_{0}+A_{1}} \leq \max _{j=0,1}\left[\sup _{t}\left\|\mathbf{e}^{(j+i t)^{2}}\left[f_{k}(j+i t)-f_{l}(j+i t)\right]\right\|_{A_{j}}\right]
$$

Como $\left\{f_{k}(z)\right\}_{k=1}^{\infty}$ é uma seqüência de Cauchy, obtemos por (4.6) que $\left\{\mathbf{e}^{z^{2}} f_{k}(z)\right\}_{k=1}^{\infty}$ está em $\bar{S}$ e com relação a $A_{0}+A_{1}$ é uma seqüência convergente. Se o limite é denotado por $\mathbf{e}^{z^{2}} f(z)$, então para $z=j+i t$ a função $f(z)$ coincide com a função $f(j+i t)$ definida acima. Então $f(z)$ é $\left(A_{0}+A_{1}\right)$-contínua em $\bar{S}$ e $\left(A_{0}+A_{1}\right)$-analítica em $S$. Agora, usando a transformação $t-\operatorname{Im} z_{0}=\tau$ 
em (4.4) e com a ajuda de (4.5), temos

$$
\begin{aligned}
\mathbf{e}^{-|\gamma|\left|\operatorname{Im} z_{0}\right|}\left\|f\left(z_{0}\right)\right\| A_{0}+A_{1} & \leq \max _{j=0,1} \sup _{t} \mathbf{e}^{-|\gamma|\left|\operatorname{Im} z_{0}\right|}\left\|\mathbf{e}^{\delta\left(j+i t-z_{0}\right)^{2}} f(j+i t)\right\|_{A_{0}+A_{1}} \\
& =\max _{j=0,1} \sup _{t} \mathbf{e}^{-|\gamma|\left|\operatorname{Im} z_{0}\right|} \mathbf{e}^{\delta\left(j-\operatorname{Re} z_{0}\right)^{2}} \mathbf{e}^{-\delta\left(t-\operatorname{Im} z_{0}\right)^{2}}\|f(j+i t)\|_{A_{0}+A_{1}} \\
& \leq \max _{j=0,1} \sup _{\tau} \mathbf{e}^{-|\gamma|\left|\operatorname{Im} z_{0}\right|-\delta \tau^{2}}\left\|f\left(j+i\left(\tau+\operatorname{Im} z_{0}\right)\right)\right\|_{A_{0}+A_{1}} \\
& \leq \max _{j=0,1} \sup _{\tau} \mathbf{e}^{-|\gamma|\left|\tau+\operatorname{Im} z_{0}\right|}\left\|f\left(j+i\left(\tau+\operatorname{Im} z_{0}\right)\right)\right\|_{A_{0}+A_{1}} \\
& \leq \max _{j=0,1} \sup _{\tau} \mathbf{e}^{-|\gamma \gamma|\left|\tau+\operatorname{Im} z_{0}\right|}\left\|\left(f_{k}-f\right)\left(j+i\left(\tau+\operatorname{Im} z_{0}\right)\right)\right\|_{A_{0}+A_{1}} \\
& +\max _{j=0,1} \sup _{\tau} \mathbf{e}^{-|\gamma|\left|\tau+\operatorname{Im} z_{0}\right|}\left\|f_{k}\left(j+i\left(\tau+\operatorname{Im} z_{0}\right)\right)\right\|_{A_{0}+A_{1}} \\
& \leq c .
\end{aligned}
$$

Agora, por (4.5), obtemos que $f(z) \in F\left(A_{0}, A_{1}, \gamma\right)$ é o limite da seqüência $\left\{f_{k}(z)\right\}_{k=1}^{\infty}$.

Passo2. Se a seqüência de Cauchy $\left\{f_{k}(z)\right\}_{k=1}^{\infty}$ do primeiro passo pertence a $F_{-}\left(A_{0}, A_{1}, \gamma\right)$, então

$$
\begin{aligned}
\mathbf{e}^{-\gamma|t|}\|f(j+i t)\|_{A_{j}} & \leq \mathbf{e}^{-\gamma|t|}\left\|f(j+i t)-f_{k}(j+i t)\right\|_{A_{j}}+\mathbf{e}^{-\gamma|t|}\left\|f_{k}(j+i t)\right\|_{A_{j}} \\
& \leq\left\|f-f_{k}\right\|_{F(\gamma)}+\mathbf{e}^{-\gamma|t|}\left\|f_{k}(j+i t)\right\|_{A_{j}}
\end{aligned}
$$

Passando limsup na desigualdade acima, obtemos que

$$
\lim _{|t| \rightarrow \infty} \mathbf{e}^{-\gamma|t|}\|f(j+i t)\|_{A_{j}}=0
$$

e assim $f \in F_{-}\left(A_{0}, A_{1}, \gamma\right)$. Resulta que $F_{-}\left(A_{0}, A_{1}, \gamma\right)$ é também um espaço de Banach. Isto encerra a prova de $a$ ).

Passo3. Se $f(z) \in F_{-}\left(A_{0}, A_{1}, \gamma\right)$, então $\mathbf{e}^{\delta z^{2}} f(z)$ também pertence a $F_{-}\left(A_{0}, A_{1}, \gamma\right)$ se $\delta>0$. De fato,

$$
\begin{aligned}
\lim _{|t| \rightarrow \infty} \mathbf{e}^{-\gamma|t|}\left\|\mathbf{e}^{\delta(j+i t)^{2}} f(j+i t)\right\|_{A_{j}} & \leq \lim _{|t| \rightarrow \infty} \mathbf{e}^{-\gamma|t|} \mathbf{e}^{\delta j^{2}} \mathbf{e}^{-\delta t^{2}}\|f(j+i t)\|_{A_{j}} \\
& \leq c \lim _{|t| \rightarrow \infty} \mathbf{e}^{-\gamma|t|}\|f(j+i t)\|_{A_{j}}=0
\end{aligned}
$$

ainda,

$$
\begin{aligned}
\left\|\mathbf{e}^{\delta z^{2}} f(z)\right\|_{A_{0}+A_{1}} & \leq \mathbf{e}^{\delta j^{2}-\delta t^{2}}\|f(z)\|_{A_{0}+A_{1}} \\
& \leq c_{1} \mathbf{e}^{|\gamma||\operatorname{Im} z|} \mathbf{e}^{-|\gamma||\operatorname{Im} z|}\|f(z)\|_{A_{0}+A_{1}} \\
& \leq c \mathbf{e}^{|\gamma||\operatorname{Im} z|}
\end{aligned}
$$

as outras propriedade são diretas e assim $\mathbf{e}^{\delta z^{2}} f(z) \in F_{-}\left(A_{0}, A_{1}, \gamma\right)$. Ainda, $\mathbf{e}^{\delta z^{2}} f(z)$ converge em $F_{-}\left(A_{0}, A_{1}, \gamma\right)$ para $f(z)$ se $\delta \rightarrow 0$. De fato,

$$
\begin{aligned}
\left\|\mathbf{e}^{\delta z^{2}} f(z)-f(z)\right\|_{F(\gamma)} & =\max _{j=0,1} \sup _{t} \mathbf{e}^{-\gamma|t|}\left\|\mathbf{e}^{\delta(j+i t)} f(j+i t)-f(j+i t)\right\|_{A_{j}} \\
& =\max _{j=0,1} \sup _{t \in[-T, T]} \mathbf{e}^{-\gamma|t|}\left|\mathbf{e}^{\delta(j+i t)}-1\right|\|f(j+i t)\|_{A_{j}} \\
& =\left|\mathbf{e}^{\delta(j+i t)}-1\right| \max _{j=0,1} \sup _{t \in[-T, T]} \mathbf{e}^{-\gamma|t|}\|f(j+i t)\|_{A_{j}} \stackrel{\delta \rightarrow 0}{\longrightarrow} 0 .
\end{aligned}
$$


Portanto é suficiente aproximar $\mathbf{e}^{\delta z^{2}} f(z)$ em $F_{-}\left(A_{0}, A_{1}, \gamma\right)$. Seja $g(z)=\mathbf{e}^{\delta z^{2}} f(z)$ e defina

$$
g_{n}(z)=\sum_{j=-\infty}^{\infty} g(z+2 \pi i j n), \quad \infty>n \geq 1
$$

Esta série é absolutamente convergente. Vejamos, observe que

$$
\begin{aligned}
\|g(z+2 \pi i j n)\| & =\left\|\mathbf{e}^{\delta(z+2 \pi i j n)^{2}} f(z+2 \pi i j n)\right\| \\
& =\left\|\mathbf{e}^{\delta z^{2}+\delta z 2 \pi i j n-\delta(2 \pi i j n)^{2}} f(z+2 \pi i j n)\right\| \\
& =\left|\mathbf{e}^{2 i \delta \operatorname{Re} z \pi j n-2 \delta \operatorname{Im} z \pi j n}\right| \mathbf{e}^{-\delta(2 \pi i j n)^{2}}\left\|e^{\delta z^{2}} f(z+2 \pi i j n)\right\| \\
& =\mathbf{e}^{-\delta(2 \pi j n)^{2}} \mathbf{e}^{\delta(-\operatorname{Im} z 2 \pi n j)}\left\|\mathbf{e}^{\delta z^{2}} f(z+2 \pi i j n)\right\| \\
& =\mathbf{e}^{-\delta(2 \pi j n)^{2}} \mathbf{e}^{\delta \frac{(2 \pi j n)^{2}}{2}} \mathbf{e}^{-\delta \frac{(\operatorname{Im} z)^{2}}{2}} \mathbf{e}^{\delta(\operatorname{Re} z)^{2}}\|f(z+2 \pi i j n)\| \\
& \leq c \mathbf{e}^{-\delta \frac{(2 \pi j n)^{2}}{2}} \mathbf{e}^{|\gamma||\operatorname{Im} z+2 \pi j n|} \mathbf{e}^{-|\gamma||\operatorname{Im} z+2 \pi j n|} \mathbf{e}^{-\delta \frac{(\operatorname{Im} z)^{2}}{2}}\|f(z+2 \pi i j n)\| \\
& \leq c \mathbf{e}^{-\delta \frac{(2 \pi j n)^{2}}{2}} \mathbf{e}^{|\gamma||\operatorname{Im} z|} \mathbf{e}^{|\gamma||2 \pi j n|} \mathbf{e}^{-\delta \frac{(\operatorname{Im} z)^{2}}{2}} \\
& =c \mathbf{e}^{\left(|\gamma||\operatorname{Im} z|-\delta \frac{(\operatorname{Im} z)^{2}}{2}\right)} \mathbf{e}^{|\gamma||2 \pi j n|-\delta \frac{(2 \pi j n)^{2}}{2}} \\
& \leq c \mathbf{e}^{-\delta \frac{(2 \pi j n)^{2}}{6}} .
\end{aligned}
$$

Como a série $\sum_{j=-\infty}^{\infty} \mathbf{e}^{-\frac{(2 \pi j n)^{2}}{6}}$ é convergente, segue que $\sum_{j=-\infty}^{\infty} g(z+2 \pi i j n)$ é absolutamente convergente. Note que $g_{n}(z)$ tem valores em $A_{0}+A_{1}$, é analítica em $0<\operatorname{Re} z<1$ e contínua em $0 \leq \operatorname{Re} z \leq 1$. Observe que $g_{n}(z)$ é periódica de período $2 \pi i n$ já que

$$
\begin{aligned}
g_{n}(z+2 \pi i n) & =\sum_{j=-\infty}^{\infty} g(z+(1+j) 2 \pi i n) \\
& =\sum_{k=-\infty}^{\infty} g(z+2 k \pi i n) \\
& =g(z)
\end{aligned}
$$

Além disso, $g_{n}(i t) \in A_{0}$ e $g_{n}(1+i t) \in A_{1}$. Segue que $g_{n}(z) \in F\left(A_{0}, A_{1}, 0\right)$ (já que $\sum_{j=-\infty}^{\infty} g(z+$ $2 \pi i j n)$ é convergente com valores em $A_{0}+A_{1}$, $\operatorname{logo} g_{n}(z)$ é limitada em $\left.A_{0}+A_{1}\right)$. Note que $g_{n} \rightarrow g$. De fato, como

$$
\|g(z+2 \pi j i n)\| \leq c \mathbf{e}^{-\delta \frac{(2 \pi j n)^{2}}{6}},
$$

$\operatorname{para} j \neq 0$,

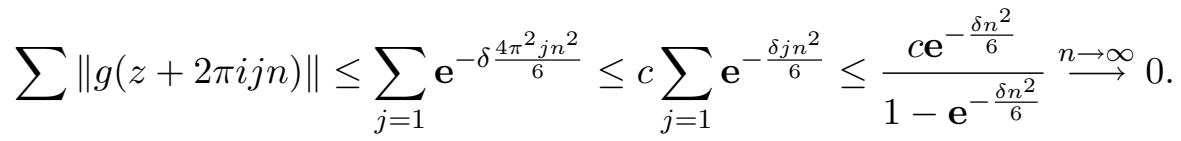

Mas,

$$
\left\|g_{n}(j+i t)-g(j+i t)\right\|_{A_{j}} \longrightarrow 0, n \longrightarrow \infty
$$


uniformemente sobre cada conjunto limitado de valores de $t$ e $\left\|g_{n}(j+i t)\right\|_{A_{j}}$ é limitado uniformemente em $n$. Como $g_{n}(z) \in F\left(A_{0}, A_{1}, 0\right)$, obtemos que $\mathbf{e}^{\eta z^{2}} g_{n}(z) \in F\left(A_{0}, A_{1}, 0\right), \eta>0$, e dado $\epsilon>0$, uma escolha apropriada de $\eta$ e $n$ nos dá

$$
\left\|\mathbf{e}^{\eta z^{2}} g_{n}(z)-g(z)\right\|_{F(0)}<\frac{\epsilon}{2}
$$

Agora, $g_{n}(z)$ tem uma representação em série de Fourier

$$
g_{n}(z)=\sum_{k=-\infty}^{\infty} a_{k n} \mathbf{e}^{\frac{k z}{n}}, \quad z=s+i t, \quad 0<s<1
$$

onde

$$
a_{k n}(s)=\frac{1}{2 \pi n m} \int_{-\pi n m}^{\pi n m} g_{n}(s+i t) \mathbf{e}^{\frac{-k(s+i t)}{n}} d t, \quad m=0,1, \ldots
$$

Pela periodicidade, o valor da integral na igualdade acima é independente de $m$. Ela também é independente de $s$. De fato, o integrando é analítico e limitado em $A_{0}+A_{1}$ e observe que $t m \stackrel{m \rightarrow \infty}{\longrightarrow} \infty$. Faça $t m \bmod 2 \pi n=t_{m} \in[-\pi n, \pi n]$, como $[-\pi n, \pi m]$ é compacto, então existe uma subseqüência de $t_{m}$, que denotaremos por $t_{m}$ convergente, $t_{m} \longrightarrow t$.

Então,

$$
g_{n}\left(s+i t_{m}\right)=\sum_{j=-\infty}^{\infty} g\left(s+i t_{m}+2 \pi i j n\right) \stackrel{m \rightarrow \infty}{\longrightarrow} \sum_{j=-\infty}^{\infty} g(s+i t+2 \pi i j n)=g(s+i t)
$$

Então, dados $s$ e $s^{\prime}$, temos

$$
\begin{aligned}
\left\|a_{k n}(s)-a_{k n}\left(s^{\prime}\right)\right\| & =\| \frac{1}{2 \pi n m} \int_{-\pi n m}^{\pi n m}\left[g_{n}(s+i t) \mathbf{e}^{-\frac{k(s+i t)}{n}}-g_{n}\left(s^{\prime}+i t\right) \mathbf{e}^{\left.-\frac{k\left(s^{\prime}+i t\right)}{n}\right] d t \|}\right. \\
& =\left\|\frac{1}{2 \pi n} \int_{-\pi n}^{\pi n}\left[g_{n}\left(s+i t_{m}\right) \mathbf{e}^{-\frac{k s}{n}}-g_{n}\left(s^{\prime}+i t_{m}\right) \mathbf{e}^{-\frac{k s^{\prime}}{n}}\right]\left[\cos \frac{k t m}{n}-i \operatorname{sen} \frac{k t m}{n}\right] d t\right\| \\
& =\frac{1}{2 \pi n} \| \int_{-\pi n}^{\pi n}\left(\left[\left[g_{n}\left(s+i t_{m}\right)-g(s+i t)\right] \mathbf{e}^{-\frac{k s}{n}}\right.\right. \\
& \left.-\left[g_{n}\left(s^{\prime}+i t_{m}\right)-g\left(s^{\prime}+i t\right)\right] \mathbf{e}^{-\frac{k s^{\prime}}{n}}\right] \\
& \left.+\left(g(s+i t) \mathbf{e}^{-\frac{k s}{n}}-g\left(s^{\prime}+i t\right) \mathbf{e}^{-\frac{k s^{\prime}}{n}}\right)\right)\left[\cos \frac{k t m}{n}-i \operatorname{sen} \frac{k t m}{n}\right] d t \| \\
& =\frac{1}{2 \pi n}\left[\int_{-\pi n}^{\pi n}\left\|g_{n}\left(s+i t_{m}\right)-g(s+i t)\right\| \mathbf{e}^{-\frac{k s}{n}} d t\right. \\
& +\int_{-\pi n}^{\pi n}\left\|g_{n}\left(s^{\prime}+i t_{m}\right)-g\left(s^{\prime}+i t\right)\right\| \mathbf{e}^{-\frac{k s^{\prime}}{n}} d t \\
& +\| \int_{-\pi n}^{\pi n}\left(g(s+i t) \mathbf{e}^{-\frac{k s}{n}}-g\left(s^{\prime}+i t\right) \mathbf{e}^{-\frac{k s^{\prime}}{n}}\right) \cos \frac{k t m}{n} d t \\
& +\int_{-\pi n}^{\pi n}\left(g(s+i t) \mathbf{e}^{-\frac{k s}{n}}-g\left(s^{\prime}+i t\right) \mathbf{e}^{-\frac{k s^{\prime}}{n}}\right) i \operatorname{sen} \frac{k t m}{n} d t \| \stackrel{m \rightarrow \infty}{\longrightarrow} 0 .
\end{aligned}
$$


A última norma converge a zero pelo Teorema de Riemann-Lebesgue. Então os valores da integral para dois valores de $s$ diferem muito pouco, se $m$ é tomado grande o suficiente. Então a integral tem o mesmo valor para dois valores de $s$. Com isso,

$$
a_{k n}=\frac{1}{2 \pi n} \int_{-\pi n}^{\pi n} g_{n}(j+i t) \mathbf{e}^{\frac{-k(j+i t)}{n}} d t, \quad j=0,1 .
$$

Agora, $g_{n}(i t) \in A_{0}$ e é $A_{0}$-contínua, o que implica que o valor da integral para $j=0$ está em $A_{0}$, ou seja, $a_{k n} \in A_{0}$. Similarmente, $g_{n}(1+i t) \in A_{1}$ e é $A_{1}$-contínua, então para $j=1, a_{k n} \in A_{1}$, concluímos então que $a_{k n} \in A_{0} \cap A_{1}$. Considere agora as $(C, 1)$ médias da série (4.8). Então temos

$$
\begin{aligned}
\sigma_{m}\left(g_{n}, z\right) & =\sum_{k=-m}^{m}\left(1-\frac{|k|}{m+1}\right) a_{k n} \mathbf{e}^{\frac{k z}{n}} \\
& =\frac{1}{2 \pi} \int_{-\pi n}^{\pi n} g_{n}(z+i t) K_{m}\left(\frac{t}{n}\right) \frac{d t}{n}
\end{aligned}
$$

onde $K_{m}(s)$ é o núcleo de Fejér. Vamos mostrar que as igualdades acima se verificam. Considere as $(C, 1)$ médias da série $(4.8)$, denotadas por $\sigma_{m}$, então

$$
\sigma_{m}=\frac{1}{m+1} \sum_{k=0}^{m} S_{k}
$$

onde $S_{m}=\sum_{k=-m}^{m} a_{k n} \mathbf{e}^{\frac{k z}{n}}$. Então, denotando $a_{k n} \mathbf{e}^{\frac{k z}{n}}$ por $x_{k}$, temos

$$
\left\{\begin{array}{l}
S_{0}=x_{0} \\
S_{1}=x_{-1}+x_{0}+x_{1} \\
S_{2}=x_{-2}+x_{-1}+x_{0}+x_{1}+x_{2} \\
\vdots \\
S_{m}=x_{-m}+\ldots+x_{-1}+x_{0}+x_{1}+\ldots+x_{m}
\end{array}\right.
$$

então, somando, obtemos que

$$
[m-(m-1)] x_{-m}+\ldots+m x_{-1}+(m+1) x_{0}+m x_{1}+\ldots+[m-(m-1)] x_{m}
$$

Assim,

$$
\frac{1}{m+1} \sum_{k=0}^{m} S_{k}=\frac{1}{m+1} \sum_{k=0}^{m}(m+1-|k|) x_{k}=\sum_{k=0}^{m}\left[1-\frac{|k|}{m+1}\right] a_{k n} \mathbf{e}^{\frac{k z}{n}}
$$

Por outro lado, se $D_{n}(t)$ denota os núcleos de Dirichlet dados por

$$
D_{n}(t)=\sum_{k=-n}^{n} \mathbf{e}^{i k t}= \begin{cases}1, & n=0 \\ 1+2 \sum_{k=1}^{n} \cos k t, & n \in \mathbb{N}\end{cases}
$$


então, claramente $D_{n}(t)$ é uma função par. Agora, se $z=x+i y$, fazendo $s=x$, obtemos

$$
\begin{aligned}
S_{m}\left(g_{n}\right) & =\sum_{k=-m}^{m} a_{k n} \mathbf{e}^{\frac{k z}{n}}=\sum_{k=-m}^{m} \frac{1}{2 \pi n} \int_{-\pi n}^{\pi n} g_{n}(x+i t) \mathbf{e}^{\frac{-k(x+i t)}{n}} \mathbf{e}^{\frac{k x}{n}+\frac{i k y}{n}} d t \\
& =\frac{1}{2 \pi n} \sum_{k=-m}^{m} \int_{-\pi m}^{\pi n} g_{n}(x+i t) \mathbf{e}^{\frac{i k}{n}(y-t)} d t \\
& =\frac{1}{2 \pi n} \int_{-\pi n}^{\pi n} g_{n}(x+i y-i t) \sum_{k=-m}^{m} \mathbf{e}^{i k \frac{t}{n}} d t \\
& =\frac{1}{2 \pi} \int_{-\pi n}^{\pi n} g_{n}(z-i t) D_{k}\left(\frac{t}{n}\right) \frac{d t}{n} .
\end{aligned}
$$

Assim,

$$
\sigma_{m}\left(g_{n}, z\right)=\frac{1}{2 \pi} \int_{-\pi n}^{\pi n} g_{n}(z-i t) K_{m}\left(\frac{t}{n}\right) \frac{d t}{n} .
$$

Seguindo, da $A_{0}$-continuidade de $g_{n}(i t)$, temos

$$
\left\|\sigma_{m}\left(g_{n}, i t\right)-g_{n}(i t)\right\|_{A_{0}} \longrightarrow 0, \quad m \rightarrow \infty
$$

uniformemente em $t$ para cada $n$. E da $A_{1}$-continuidade de $g_{n}(1+i t)$, obtemos

$$
\left\|\sigma_{m}\left(g_{n}, 1+i t\right)-g_{n}(1+i t)\right\|_{A_{1}} \longrightarrow 0, \quad m \rightarrow \infty
$$

uniformemente em $t$ para cada $n$. Assim, para $\eta>0$ e $n$ natural, segue que

$$
\left\|\mathbf{e}^{\eta z^{2}}\left[\sigma_{m}\left(g_{n}, z\right)-g_{n}(z)\right]\right\|_{F\left(A_{0}, A_{1}, 0\right)} \rightarrow 0, \quad m \rightarrow \infty .
$$

Então para $s, m$ e $n$ apropriados, temos por (4.7) e (4.9) que

$$
\left\|\mathbf{e}^{\eta z^{2}} \sigma_{m}\left(g_{n}, z\right)-g(z)\right\|_{F\left(A_{0}, A_{1}, 0\right)}<\epsilon
$$

Isto encerra a prova.

\subsection{Os Espaços $\left[A_{0}, A_{1}\right]_{\theta, \gamma}$ e $\left[A_{0}, A_{1}\right]_{\theta, \gamma,-}$}

Seja $\left\{A_{0}, A_{1}\right\}$ um par de interpolação, $\gamma$ um número real e $0<\theta<1$.

\section{Definição 4.4.1}

$$
\begin{gathered}
{\left[A_{0}, A_{1}\right]_{\theta, \gamma}=\left\{a: a \in A_{0}+A_{1}, \exists f(z) \in F\left(A_{0}, A_{1}, \gamma\right) \operatorname{com} f(\theta)=a\right\},} \\
{\left[A_{0}, A_{1}\right]_{\theta, \gamma,-}=\left\{a: a \in A_{0}+A_{1}, \exists f(z) \in F_{-}\left(A_{0}, A_{1}, \gamma\right) \operatorname{com} f(\theta)=a\right\},}
\end{gathered}
$$




$$
\|a\|_{\left[A_{0}, A_{1}\right]_{\theta, \gamma}}=\|a\|_{\left[A_{0}, A_{1}\right]_{\theta, \gamma,-}}=\inf _{f(\theta)=a}\|f(z)\|_{F(\gamma)} .
$$

$O$ infimo é tomado sobre todas as $f \in F\left(A_{0}, A_{1}, \gamma\right)$ com $f(\theta)=$ a ou toda $f \in F_{-}\left(A_{0}, A_{1}, \gamma\right)$ com $f(\theta)=$ a respectivamente .

Lema 4.4.1 Os espaços $\left[A_{0}, A_{1}\right]_{\theta, \gamma}$ e $\left[A_{0}, A_{1}\right]_{\theta, \gamma,-}$ equipados com a norma (4.12) são espaços vetoriais normados.

Prova. Mostremos que (4.12) é realmente uma norma. De fato,

i) Se $\|a\|_{\left[A_{0}, A_{1}\right]_{\theta, \gamma}}=0$, então $\inf _{f(\theta)=a}\|f(z)\|_{F(\gamma)}=0$, assim, existe $\left\{f_{n}(z)\right\} \subset F(\gamma)$ tal que $f_{n}(z) \rightarrow 0$ de maneira que $f_{n}(z)=a, \forall n, \operatorname{logo}, a=0$.

ii) Se $\lambda \in \mathbb{R}$, então

$$
\begin{aligned}
\|\lambda a\|_{\left[A_{0}, A_{1}\right]_{\theta, \gamma}} & =\inf _{f(\theta)=\lambda a}\|f(z)\|_{F(\gamma)}=\inf _{g(\theta)=a}\|\lambda g(z)\|_{F(\gamma)} \\
& =|\lambda| \inf _{g(\theta)=a}\|g(z)\|_{F(\gamma)}=|\lambda|\|a\|_{\left[A_{0}, A_{1}\right]_{\theta, \gamma}}
\end{aligned}
$$

iii) Se $a, b \in\left[A_{0}, A_{1}\right]_{\theta, \gamma}$, então

$$
\begin{aligned}
\|a+b\|_{\left[A_{0}, A_{1}\right]_{\theta, \gamma}} & =\inf _{f(\theta)=a+b}\|f(z)\|_{F(\gamma)} \leq \inf _{f_{1}(\theta)=a}\left\|f_{1}(z)\right\|_{F(\gamma)}+\inf _{f_{2}(\theta)=b}\left\|f_{2}(z)\right\|_{F(\gamma)} \\
& =\|a\|_{\left[A_{0}, A_{1}\right]_{\theta, \gamma}}+\|b\|_{\left[A_{0}, A_{1}\right]_{\theta, \gamma}}
\end{aligned}
$$

Teorema 4.4.1 Os espaços $\left[A_{0}, A_{1}\right]_{\theta, \gamma} e\left[A_{0}, A_{1}\right]_{\theta, \gamma,-}$ equipados com a norma (4.12) são espaços de Banach. Todos os espaços de Banach $\left[A_{0}, A_{1}\right]_{\theta, \gamma} e\left[A_{0}, A_{1}\right]_{\theta, \delta,-},-\infty<\gamma<\delta<\infty$, coincidem (normas equivalentes).

Prova. Considere a aplicação de $F\left(A_{0}, A_{1}, \gamma\right)$ em $\left[A_{0}, A_{1}\right]_{\theta, \gamma}$, dada por

$$
f \rightarrow f(\theta)
$$

Esta aplicação é linear, sobrejetora e contínua,

$$
\|f(\theta)\|_{\left[A_{0}, A_{1}\right]_{\theta, \gamma}}=\inf _{h(\theta)=f(\theta)}\|h(z)\|_{F(\gamma)} \leq\|f\|_{F(\gamma)} .
$$

O núcleo desta aplicação é

$$
\left.N_{\theta}=\left\{f(z): f(z) \in F\left(A_{0}, A_{1}, \gamma\right), f(\theta)=0\right)\right\}
$$

que é fechado. Então, segue que

$$
\frac{F\left(A_{0}, A_{1}, \gamma\right)}{N_{\theta}} \cong\left[A_{0}, A_{1}\right]_{\theta, \gamma} .
$$


Segue que $\frac{F\left(A_{0}, A_{1}, \gamma\right)}{N_{\theta}}$ é isometricamente isomorfo a $\left[A_{0}, A_{1}\right]_{\theta, \gamma}$ e assim $\left[A_{0}, A_{1}\right]_{\theta, \gamma}$ é um espaço de Banach. Da mesma maneira, se mostra que $\left[A_{0}, A_{1}\right]_{\theta, \gamma,-}$ é um espaço de Banach. Se $f(\theta)=$ $a, f(z) \in F\left(A_{0}, A_{1}, \gamma\right)$, então $\mathbf{e}^{(z-\theta)^{2}} f(z)$ pertence a todos os espaços $\left[A_{0}, A_{1}\right]_{\theta, \delta}$ e $\left[A_{0}, A_{1}\right]_{\theta, \delta,-}$, e ainda, $g(\theta)=a$. Portanto, todos os espaços considerados coincidem no sentido de conjuntos. Desde que as normas são comparáveis, segue do Teorema do Gráfico Fechado que as normas destes espaços são equivalentes.

Convenção. Espaços de Banach diferindo somente por normas equivalentes serão considerados iguais. Neste sentido, vamos identificar os espaços $\left[A_{0}, A_{1}\right]_{\theta, \gamma}$ e $\left[A_{0}, A_{1}\right]_{\theta, \delta,-},-\infty<\gamma<\delta<\infty$ e escrever simplesmente $\left[A_{0}, A_{1}\right]_{\theta}$. Para fixar a notação, $\left[A_{0}, A_{1}\right]_{\theta}=\left[A_{0}, A_{1}\right]_{\theta, 0}$ (se nada for dito).

Teorema 4.4.2 a) $\left[A_{0}, A_{1}\right]_{\theta}, 0<\theta<1$, é um espaço de interpolação (com respeito a $A_{0} e$ $A_{1}$ ). O funtor de interpolação correspondente é exato e de tipo $\theta$.

b)

$$
\left[A_{0}, A_{1}\right]_{\theta}=\left[A_{0}, A_{1}\right]_{1-\theta}
$$

c) $A_{0} \cap A_{1}$ é denso em $\left[A_{0}, A_{1}\right]_{\theta}$.

d) Se $A_{0} \subset A_{1}$, então para $0<\theta<\tilde{\theta}<1$,

$$
A_{0} \subset\left[A_{0}, A_{1}\right]_{\theta} \subset\left[A_{0}, A_{1}\right]_{\tilde{\theta}} \subset A_{1} .
$$

e) $S e A_{0}=A_{1}$ então $\left[A_{0}, A_{1}\right]_{\theta}=A_{0}=A_{1}$.

f) Existe um número positivo $c_{\theta}, 0<\theta<1$, tal que para todo a $\in A_{0} \cap A_{1}$

$$
\|a\|_{\left[A_{0}, A_{1}\right]_{\theta}} \leq c_{\theta}\|a\|_{A_{0}}^{1-\theta}\|a\|_{A_{1}}^{\theta} .
$$

g) Se $\AA_{j}$ denota o completamento de $A_{0} \cap A_{1}$ em $A_{j}, j=0,1$, então

$$
\left[A_{0}, A_{1}\right]_{\theta}=\left[\AA_{0}, A_{1}\right]_{\theta}=\left[A_{0}, \AA_{1}\right]_{\theta}=\left[\AA_{0}, \AA_{1}\right]_{\theta} .
$$

Prova. Passo1. Mostremos que

$$
A_{0} \cap A_{1} \subset\left[A_{0}, A_{1}\right]_{\theta} \subset A_{0}+A_{1}
$$

Seja $a \in A_{0} \cap A_{1}$. Então a função constante $f(z)=a$ pertence a $F\left(A_{0}, A_{1}, 0\right)$. Portanto, $a \in\left[A_{0}, A_{1}\right]_{\theta} \mathrm{e}$

$$
\|a\|_{\left[A_{0}, A_{1}\right]_{\theta}}=\inf _{f(\theta)=a}\|f(z)\|_{F(0)} \leq \max _{j=0,1}\|a\|_{A_{j}}=\|a\|_{A_{0} \cap A_{1}} .
$$


Claramente $\left[A_{0}, A_{1}\right]_{\theta} \subset A_{0}+A_{1}$, seja $a \in\left[A_{0}, A_{1}\right]_{\theta}$ e $f(\theta)=a, f(z) \in F\left(A_{0}, A_{1}, 0\right)$. Por (4.4), obtemos

$$
\|a\|_{A_{0}+A_{1}} \leq \max _{j=0,1}\left[\sup _{t}\|f(j+i t)\|_{A_{j}}\right]=\|f(z)\|_{F(0)} .
$$

A construção do ínfimo nos dá que

$$
\|a\|_{A_{0}+A_{1}} \leq\|a\|_{\left[A_{0}, A_{1}\right]_{\theta}}
$$

o que prova (4.16).

Passo2. Para provar que o funtor de interpolação pertencente a $\left[A_{0}, A_{1}\right]_{\theta}$ é exato e de tipo $\theta$, começamos com $a \in\left[A_{0}, A_{1}\right]_{\theta}$ e $T \in L\left(\left\{A_{0}, A_{1}\right\},\left\{B_{0}, B_{1}\right\}\right)$. Seja $f(z) \in F\left(A_{0}, A_{1}, 0\right)$ e $f(\theta)=a$. Então

$$
g(z)=\left(\frac{\|T\|_{L\left(A_{0}, B_{0}\right)}}{\|T\|_{L\left(A_{1}, B_{1}\right)}}\right)^{z-\theta} T f(z) \in F\left(B_{0}, B_{1}, 0\right), \quad g(\theta)=T a .
$$

(Se um dos números $\|T\|_{L\left(A_{0}, B_{0}\right)}$ ou $\|T\|_{L\left(A_{1}, B_{1}\right)}$ é zero, então ele é substituído por $\epsilon>0$, depois fazemos $\epsilon \downarrow 0$ ). Ainda,

$$
\begin{aligned}
\|g(z)\|_{F\left(B_{0}, B_{1}, 0\right)} & =\left\|\left(\frac{\|T\|_{L\left(A_{0}, B_{0}\right)}}{\|T\|_{L\left(A_{1}, B_{1}\right)}}\right)^{z-\theta} T f(z)\right\|_{F\left(B_{0}, B_{1}, 0\right)} \\
& =\max _{j=0,1}\left(\sup _{t}\left\|\left(\frac{\|T\|_{L\left(A_{0}, B_{0}\right)}}{\|T\|_{L\left(A_{1}, B_{1}\right)}}\right)^{(j+i t-\theta)} T f(j+i t)\right\|_{B_{j}}\right) \\
& \leq\left[\frac{\|T\|_{L\left(A_{0}, B_{0}\right)}^{1-\theta}}{\|T\|_{L\left(A_{1}, B_{1}\right)}^{-\theta}} \sup _{t}\|f(i t)\|_{A_{0}}, \frac{\|T\|_{L\left(A_{0}, B_{0}\right)}^{1-\theta}}{\|T\|_{L\left(A_{1}, B_{1}\right)}^{-\theta}} \sup _{t}\|f(1+i t)\|_{A_{1}}\right] \\
& =\|T\|_{L\left(A_{0}, B_{0}\right)}^{1-\theta}\|T\|_{L\left(A_{1}, B_{1}\right)}^{\theta} \max _{j=0,1} \sup _{t}\|f(j+i t)\|_{A_{j}} \\
& \leq\|T\|_{L\left(A_{0}, B_{0}\right)}^{1-\theta}\|T\|_{L\left(A_{1}, B_{1}\right)}^{\theta}\|f(z)\|_{F(0)} .
\end{aligned}
$$

Assim,

$$
\|T a\|_{\left[B_{0}, B_{1}\right]_{\theta}}=\inf _{f(\theta)=T a}\|f(z)\|_{F(0)} \leq\|g(z)\|_{F\left(B_{0}, B_{1}, 0\right)} \leq\|T\|_{L\left(A_{0}, B_{0}\right)}^{1-\theta}\|T\|_{L\left(A_{1}, B_{1}\right)}^{\theta}\|a\|_{\left[A_{0}, A_{1}\right]_{\theta}} .
$$

Passo3. Se $f(z) \in F\left(A_{0}, A_{1}, 0\right)$, então $g(z)=f(1-z) \in F\left(A_{1}, A_{0}, 0\right)$ e

$$
\|a\|_{\left[A_{0}, A_{1}\right]_{\theta}}=\inf _{f(\theta)=a}\|f(z)\|_{F\left(A_{0}, A_{1}, 0\right)}=\inf _{g(1-\theta)=a}\|g(z)\|_{F\left(A_{1}, A_{0}, 0\right)}=\|a\|_{\left[A_{1}, A_{0}\right]_{1-\theta}} .
$$

Isto prova $b$ ).

Passo4. Tome $a \in\left[A_{0}, A_{1}\right]_{\theta}$, então existe $f(z) \in F\left(A_{0}, A_{1}, 0\right)$ com $f(\theta)=a$. Então existe $g$ no gerado pelas funções $\mathbf{e}^{\delta z^{2}+\lambda z} a$ dado pelo Teorema 4.3 .2 item $b$ ) tal que

$$
\|f-g\|_{F}<\epsilon
$$


Portanto,

$$
\|a-g(\theta)\|_{\left[A_{0}, A_{1}\right]_{\theta}}=\inf _{h(z)=a-g(\theta)}\|h(z)\|_{F} \leq\|f-g\|_{F}<\epsilon .
$$

Como $g(\theta) \in A_{0} \cap A_{1}$ segue que $\overline{A_{0} \cap A_{1}}=\left[A_{0}, A_{1}\right]_{\theta}$, o que prova o item $c$ ).

Passo5. Seja $A_{0} \subset A_{1}$. Por (4.16), obtemos que

$$
A_{0} \subset\left[A_{0}, A_{1}\right]_{\tilde{\theta}} \subset A_{1}, \quad 0<\tilde{\theta}<1
$$

Particularmente, $\left\{A_{0},\left[A_{0}, A_{1}\right]_{\tilde{\theta}}\right\}$ é um par de interpolação. Seja $0<\theta<\tilde{\theta}<1$ e $\theta=\tilde{\theta} \lambda$, $0<\lambda<1$. Queremos mostrar que

$$
\left[A_{0}, A_{1}\right]_{\theta} \subset\left[A_{0},\left[A_{0}, A_{1}\right]_{\tilde{\theta}}\right]_{\lambda}
$$

Seja $a \in A_{0}$ e $f(z) \in F\left(A_{0}, A_{1}, 0\right)$ com $f(\theta)=a$. Deste modo é assumido que $f(z)$ é uma combinação linear de funções descritas no Teorema 4.3.2 (b). Pela definição dos espaços $\left[A_{0}, A_{1}\right]_{\theta}$, segue que

$$
\|f(\tilde{\theta}+i t)\|_{\left[A_{0}, A_{1}\right]_{\tilde{\theta}}}=\inf _{h(\tilde{\theta})=f(\tilde{\theta}+i t)}\|h(z)\|_{F(0)} \leq\|f\|_{F\left(A_{0}, A_{1}, 0\right)}, \quad-\infty<t<\infty .
$$

Fazendo $g(z)=f(\tilde{\theta} z), z \in \bar{S}$, obtemos que

$$
g(z) \in F\left(A_{0},\left[A_{0}, A_{1}\right]_{\tilde{\theta}}, 0\right)
$$

e

$$
\|g\|_{F\left(A_{0},\left[A_{0}, A_{1}\right]_{\tilde{\theta}}, 0\right)}=\max _{j=0,1}\left(\sup _{t}\|g(i t)\|_{A_{0}}, \sup _{t}\|g(1+i t)\|_{\left[A_{0}, A_{1}\right]_{\tilde{\theta}}}\right) \leq\|f\|_{F\left(A_{0}, A_{1}, 0\right)}
$$

e $g(\lambda)=f(\theta)=a$. Como $A_{0}$ é denso em $\left[A_{0}, A_{1}\right]_{\theta}$, segue (4.18). Note que

$$
\left[A_{0},\left[A_{0}, A_{1}\right]_{\tilde{\theta}}\right]_{\lambda} \subset\left[\left[A_{0}, A_{1}\right]_{\tilde{\theta}},\left[A_{0}, A_{1}\right]_{\tilde{\theta}}\right]_{\lambda}=\left[A_{0}, A_{1}\right]_{\tilde{\theta}}
$$

Então,

$$
\|a\|_{\tilde{\theta}} \leq c\|g(\lambda)\|_{\left[A_{0},\left[A_{0}, A_{1}\right]_{\tilde{\theta}}\right]_{\lambda}} \leq c\|g\|_{F\left(A_{0},\left[A_{0}, A_{1}\right]_{\tilde{\theta}}, 0\right)} \leq c\|f\|_{F\left(A_{0}, A_{1}, 0\right)}
$$

segue que

$$
\|a\|_{\left[A_{0}, A_{1}\right]_{\tilde{\theta}}} \leq c\|a\|_{\left[A_{0}, A_{1}\right]_{\theta}} .
$$

Passo6. O item e) é conseqüência de (4.16). Para provar $f$ ), fixe $a \in A_{0} \cap A_{1}, 0<\theta<1$ e considere

$$
T(\lambda)=\lambda a, \quad \lambda \in \mathbb{C}
$$


como uma transformação linear de $\mathbb{C}$ em $A_{0}$ ou $A_{1}$. Note que

$$
\|T\|_{L\left(\mathbb{C}, A_{j}\right)}=\|a\|_{A_{j}}
$$

Agora usando a), a transformação

$$
T:[\mathbb{C}, \mathbb{C}]_{\theta} \longrightarrow\left[A_{0}, A_{1}\right]_{\theta}
$$

tem norma

$$
\|T\|_{L\left([\mathbb{C}, \mathbb{C}]_{\theta},\left[A_{0}, A_{1}\right]_{\theta}\right)} \leq\|T\|_{L\left(\mathbb{C}, A_{0}\right)}^{1-\theta}\|T\|_{L\left(\mathbb{C}, A_{1}\right)}^{\theta}=\|a\|_{A_{0}}^{1-\theta}\|a\|_{A_{1}}^{\theta}
$$

e como

$$
\|T \lambda\|_{\left[A_{0}, A_{1}\right]_{\theta}}=\|\lambda a\|_{\left[A_{0}, A_{1}\right]_{\theta}}=\|\lambda\|_{[\mathbb{C}, \mathbb{C}]_{\theta}}\|a\|_{\left[A_{0}, A_{1}\right]_{\theta}},
$$

e

$$
\|T\|_{L\left([\mathbb{C}, \mathbb{C}]_{\theta},\left[A_{0}, A_{1}\right]_{\theta}\right)}=\|a\|_{\left[A_{0}, A_{1}\right]_{\theta}} \geq c^{-1}\|a\|_{\left[A_{0}, A_{1}\right]_{\theta}} .
$$

Assim, obtemos que

$$
\|a\|_{\left[A_{0}, A_{1}\right]_{\theta}} \leq c\|a\|_{A_{0}}^{1-\theta}\|a\|_{A_{0}}^{\theta}
$$

Passo7. Observe que

$$
\begin{aligned}
& A_{0} \cap A_{1} \subset \stackrel{\circ}{A}_{0} \cap A_{1} \subset\left[\stackrel{\circ}{A}_{0}, A_{1}\right]_{\theta} \subset\left[A_{0}, A_{1}\right]_{\theta} \\
& A_{0} \cap A_{1} \subset A_{0} \cap \stackrel{\circ}{A}_{1} \subset\left[A_{0}, \stackrel{\circ}{A}_{1}\right]_{\theta} \subset\left[A_{0}, A_{1}\right]_{\theta} \\
& A_{0} \cap A_{1} \subset \stackrel{\circ}{A}_{0} \cap \stackrel{\circ}{A}_{1} \subset\left[\stackrel{\circ}{A}_{0}, \stackrel{\circ}{A}_{1}\right]_{\theta} \subset\left[A_{0}, A_{1}\right]_{\theta}
\end{aligned}
$$

então $g$ ) segue de $c$ ). 


\section{Capítulo 5}

\section{Resultados Finais}

Finalmente, neste capítulo vamos relacionar os espaços de potências fracionárias com os espaços de interpolação complexa.

\subsection{Potências Fracionárias e Espaços de Interpolação}

Vamos iniciar esta seção com um importante resultado devido a H. Triebel, ver [21].

Teorema 5.1.1 Seja A um operador positivo. Suponha que exista dois números positivos $\epsilon$ e $C$ tais que $A^{i t}$ é um operador limitado para $-\epsilon \leq t \leq \epsilon e\left\|A^{i t}\right\| \leq C$. Se $\alpha$ e $\beta$ são dois números complexos, $0 \leq \operatorname{Re} \alpha<\operatorname{Re} \beta<\infty$ e $0<\theta<1$, então

$$
\left[D\left(A^{\alpha}\right), D\left(A^{\beta}\right)\right]_{\theta}=D\left(A^{\alpha(1-\theta)+\beta \theta}\right) .
$$

Prova. Primeiramente, observe que pelo Teorema 2.2.3, item vi), podemos supor sem perda de generalidade que $\alpha=0$. Então, queremos mostrar que

$$
\left[E, D\left(A^{\beta}\right)\right]_{\theta}=D\left(A^{\beta \theta}\right)
$$

Agora, se $-\infty<t<\infty$, então $|t|$ pode ser representado como $|t|=\epsilon N+\delta \operatorname{com} N=0,1, \ldots$ e $0 \leq \delta<\epsilon$. Do Teorema 2.2.3, iii) podemos encontrar $a \in D\left(A^{4}\right)$ tal que

$$
\left\|A^{i t} a\right\| \leq C^{N+1}\|a\| \leq c \mathbf{e}^{\gamma|t|}\|a\|
$$

onde $c$ e $\gamma$ são números não-negativos apropriados. (Como $C \geq 1$, segue que $\gamma \geq 0$.) Obtemos que $A^{i t}$ é um operador limitado para todo $t$, com $-\infty<t<\infty$ e ainda

$$
\left\|A^{i t}\right\| \leq c \mathbf{e}^{\gamma|t|} .
$$


Do Teorema $2.2 .3 v$ ) segue que $D\left(A^{m}\right)$ é denso em $D\left(A^{\beta}\right)$ para cada número natural $m$, com $m>\operatorname{Re} \beta$. Observe que

$$
D\left(A^{\beta}\right)=D\left(A^{\operatorname{Re} \beta}\right)
$$

para $\beta>0$. Então, podemos assumir sem perda de generalidade que $\operatorname{Re} \beta>0$ em (5.1). Agora, seja $\alpha=0, \beta>0$ e $a \in D\left(A^{m}\right)$, onde $m$ é um número natural suficientemente grande. Então, para $0<\theta<1$, obtemos que

$$
\begin{aligned}
\|a\|_{\left[E, D\left(A^{\beta}\right)\right]_{\theta}} & \leq c\left\|\mathbf{e}^{(z-\theta)^{2}} A^{-(z-\theta) \beta} a\right\|_{F\left(E, D\left(A^{\beta}\right), 0\right)} \\
& =c \max _{j=0,1}\left[\sup _{t}\left\|\mathbf{e}^{(i t-\theta)^{2}} A^{-i t \beta} A^{\theta \beta} a\right\|_{E},\right. \\
& \left.\sup _{t}\left\|\mathbf{e}^{(1+i t-\theta)^{2}} A^{-\beta} A^{-i t \beta} A^{\theta \beta} a\right\|_{D\left(A^{\beta}\right)}\right] \\
& \leq c \max _{j=0,1}\left[\sup _{t}\left\|\mathbf{e}^{(i t-\theta)^{2}} A^{-i t \beta} A^{\theta \beta} a\right\|_{E},\right. \\
& \left.\sup _{t}\left\|\mathbf{e}^{(1+i t-\theta)^{2}} A^{-i t \beta} A^{\theta \beta} a\right\|_{E}\right] \\
& \leq c\left\|A^{\theta \beta} a\right\| .
\end{aligned}
$$

Agora, lembrando que $D\left(A^{m}\right)$ é denso em $D\left(A^{\theta \beta}\right)$, tome $a \in D\left(A^{\theta \beta}\right)$, então existe $a_{n} \in D\left(A^{m}\right)$ tal que

$$
a_{n} \stackrel{D\left(A^{\theta \beta}\right)}{\longrightarrow} a
$$

então, da estimativa anterior

$$
a_{n} \stackrel{\left[E, D\left(A^{\beta}\right)\right]_{\theta}}{\longrightarrow} a
$$

como $\left[E, D\left(A^{\beta}\right)\right]_{\theta}$ é um espaço de Banach, segue que $a \in\left[E, D\left(A^{\beta}\right)\right]_{\theta}$. Mais precisamente,

$$
D\left(A^{\theta \beta}\right) \subset\left[E, D\left(A^{\beta}\right)\right]_{\theta} .
$$

Por outro lado, seja $\alpha=0, \beta>0$ e $a \in D\left(A^{m}\right)$, onde $m$ é suficientemente grande. Como $D\left(A^{m}\right)$ é denso em $D\left(A^{\beta}\right)$, segue do Teorema $\left.4.3 .2 b\right)$ que o gerado pelas funções $\mathbf{e}^{\delta z^{2}+\lambda z} b, \delta>0, \lambda$ real, $b \in D\left(A^{m}\right)$ é denso em $F_{-}\left(E, D\left(A^{\beta}\right), \eta\right)$, onde $\eta$ é um número natural arbitrário. Denotemos as combinações lineares de um número finito de funções $\mathbf{e}^{\delta_{j} z^{2}+\lambda_{j} z} b_{j}$ por $L\left(\mathbf{e}^{\delta_{j} z^{2}+\lambda_{j} z} b_{j}\right)$. Se $\gamma$ tem o mesmo significado que em (5.3), então do Teorema 4.4.1 e 4.4.2 item (e), temos

$$
\begin{aligned}
& \left\|A^{\theta \beta} a\right\| \leq c\left\|A^{\theta \beta} a\right\|_{[E, E]_{\beta, \gamma}} \\
& \leq c \inf _{L\left(\mathbf{e}^{\delta_{j} z^{2}+\lambda_{j} z} b_{j}\right)}\left\|L\left(\mathbf{e}^{\delta_{j} z^{2}+\gamma_{j} z} A^{z \beta} b_{j}\right)\right\|_{F(E, E, \beta \gamma)} \\
& \leq c \inf _{L\left(\mathbf{e}^{\delta_{j} z^{2}+\lambda_{j} z} b_{j}\right)}\left\|L\left(\mathbf{e}_{z=\theta}^{\delta_{j} z^{2}+\lambda_{j} z} b_{j}\right)\right\|_{F\left(E, D\left(A^{\beta}\right), 0\right)} \\
& =c\|a\|_{\left[E, D\left(A^{\beta}\right)\right]_{\theta}}
\end{aligned}
$$


Agora, como $D\left(A^{\beta}\right)$ é denso em $\left[E, D\left(A^{\beta}\right)\right]_{\theta}$, segue que

$$
\overline{D\left(A^{m}\right)}=\left[E, D\left(A^{\beta}\right)\right]_{\theta}
$$

já que

$$
D\left(A^{m}\right) \stackrel{d}{\hookrightarrow} D\left(A^{\beta}\right) \stackrel{d}{\hookrightarrow} D\left(A^{\theta \beta}\right) \hookrightarrow\left[E, D\left(A^{\beta}\right)\right]_{\theta}
$$

Pela cadeia acima, dado $a \in D\left(A^{\beta}\right)$, existe uma seqüência $a_{n} \in D\left(A^{m}\right)$ que converge para $a$ na norma de $D\left(A^{\beta}\right)$ e portanto $a_{n}$ também converge a a na norma de $D\left(A^{\theta \beta}\right)$. Pela estimativa (5.5), resulta que

$$
a_{n} \stackrel{\left[E, D\left(A^{\beta}\right)\right]_{\theta}}{\longrightarrow} a
$$

Lembrando que $D\left(A^{\beta}\right)$ é denso em $\left[E, D\left(A^{\beta}\right)\right]_{\theta}$, obtemos a densidade de $D\left(A^{m}\right)$ em $\left[E, D\left(A^{\beta}\right)\right]_{\theta}$. Dado $a \in\left[E, D\left(A^{\beta}\right)\right]_{\theta}$, existe $a_{n} \in D\left(A^{m}\right)$ tal que

$$
a_{n} \stackrel{\left[E, D\left(A^{\beta}\right)\right]_{\theta}}{\longrightarrow} a
$$

e da estimativa $(5.5)$

$$
a_{n} \stackrel{D\left(A^{\theta \beta}\right)}{\longrightarrow} a
$$

e do fato de $A^{\theta \beta}$ ser fechado, segue que $a \in D\left(A^{\theta \beta}\right)$. Logo,

$$
\left[E, D\left(A^{\beta}\right)\right]_{\theta} \subset D\left(A^{\theta \beta}\right)
$$

Por (5.4) e (5.6), segue o resultado.

Corolário 5.1.1 Seja $H$ um espaço de Hilbert e $A: D(A) \subset H \longrightarrow H$ um operador fechado, maximal acretivo e densamente definido, $0<\theta<1$ e $\alpha$ um complexo com $0 \leq \operatorname{Re} \alpha<\infty$, então

$$
D\left(A^{\alpha \theta}\right)=\left[H, D\left(A^{\alpha}\right)\right]_{\theta}
$$

ou seja, em espaços de Hilbert, as potências fracionárias de operadores acretivos coincidem com os espaços de interpolação complexa.

Prova. Do Teorema 3.1.2, segue que $A \in \mathcal{P} \mathcal{I} \mathcal{L}$ e o resultado segue do Teorema 5.1.1.

Teorema 5.1.2 Se H é um espaço de Hilbert e $A: D(A) \subset H \rightarrow H$ é o gerador de um semigrupo fortemente contínuo com decaimento exponencial, então

$$
D\left((-A)^{\alpha \theta}\right)=\left[H, D\left(A^{\alpha}\right)\right]_{\theta}
$$

Prova. Dos Lemas 1.2.1 e 1.3.1, existe $\|$.$\| em H$ que torna $-A$ maximal acretivo. 


\section{Referências Bibliográficas}

[1] Adams, R. A., Sobolev Spaces. Academic Press Inc., New York, 1975.

[2] Amann, H., Linear and Quasilinear Parabolic Problems. Vol. I. Birkhäuser Verlag, Basel, 1995.

[3] Amann, H., On Abstract Parabolic Fundamental Solutions. J. Math. Soc. Japan, 39 (1987), 93-116.

[4] Amann, H., Nonhomogeneous Linear and Quasilinear Elliptic and Parabolic Boundary Value Problems, in: Schmeisser/Triebel: Function Spaces, Differential Operators and Nonlinear Analysis, Teubner Texte zur Mathematik, 133 (1993), 9-126.

[5] Arrieta, J. \& Carvalho, A. N., Abstract Parabolic Problems with Critical Nonlinearities and Applications to Navier-Stokes and Heat Equations. Transactions of the American Mathematical Society, 352(1), 285-310 (2000).

[6] Bergh, J., Löfström, J., Interpolation Spaces, An Instroduction. Springer-Verlag, Berlin, 1976.

[7] Brézis, H., Análisis funcional. Teoría y aplicaciones. Alianza Editorial S. A., Madrid, 1984.

[8] Calderón, A. P., Intermediate Spaces and Interpolation. The Complex Method. Studia Mathematica. T. XXIV. (1964), 113-190.

[9] Conway, J. B., Functions of One Complex Variable. Second Edition. Springer-Verlag. New York, 1978.

[10] Czaja, R., Differential Equations with Sectorial Operator. Wydawnictwo Uniwersytetu Ślaskiego. Katowice, 2002.

[11] Dunford, N., Schwartz, J. T., Linear Operators. Part I and II. John Wiley \& Sons Inc., New York, 1988. 
[12] Folland, G. B., Real Analysis. Modern Techniques and Their Applications. John Wiley \& Sons Inc., New York, 1984.

[13] Henry, D., Geometric Theory of Semilinear Parabolic Equations. Springer-Verlag, Berlin, 1981.

[14] Henry, D., Semigroups. Course Notes. IME/USP, São Paulo, Brazil, [1981].

[15] Kato, T., Note on Fractional Powers of Linear Operators. Proc. Japan Acad., 36 (1960), 94-96.

[16] Kato, T., Fractional Powers of Dissipative Operators. J. Math. Soc. Japan, 13 (1961), 246574.

[17] Kato,T., Fractional Powers of Dissipative Operators, II. J. Math. Soc. Japan, 14 (1962), $242-248$.

[18] Krě̌n, S.G., Linear Differential Equations in Banach Space. AMS, 1971.

[19] Pazy, A., Semigroups of Linear Operators and Applications to Partial Differential Equations. Springer-Verlag, New York, 1963.

[20] Taylor, A. E., Lay, D. C., Introduction to Functional Analysis. Second Edition. John Wiley \& Sons Inc., New York, 1958.

[21] Triebel, H., Interpolation Theory, Function Spaces, Differential Operators. Johann Ambrosius Barth, Heidelberg, second edition, 1955.

[22] Yosida, K., Fractional powers of infinitesimal generators and the analyticity of the semigroups generated by them. Proc. Japan Acad., 36 (1960), 86-89.

[23] Yosida, K., Functional Analysis. Springer-Verlag, New York, 1974. 


\section{Índice Remissivo}

Categoria, 70

Espaço de Interpolação, 70

Espectro, 8

Funtor

de Interpolação, 70

de expoente $\theta, 70$

do tipo $f, 70$

exato, 70

Gerador infinitesimal, 8

Imagem numérica, 12

Operador

acretivo, 53

auto-adjunto, 11

dissipativo, 10

do tipo $(\omega, M), 42$

fechável, 12

parte, 16

positivo, 30

setorial, 22

simétrico, 11

unitário, 18

Os Espaços

$$
\begin{aligned}
& {\left[A_{0}, A_{1}\right]_{\theta, \gamma,-}, 78} \\
& {\left[A_{0}, A_{1}\right]_{\theta, \gamma}, 78} \\
& F\left(A_{0}, A_{1}, \gamma\right), 71 \\
& F_{-}\left(A_{0}, A_{1}, \gamma\right), 72
\end{aligned}
$$

Par de Interpolação, 67

Potência Fracionária, 31, 38

Potências Imaginárias Limitadas, 50

Principal, 56

Pseudo-Resolvente, 13

Resolvente, 8

Semigrupo, 7

de ângulo $\alpha, 37$

de contrações, 10

\section{Teorema}

de Hille-Yosida, 9

de Hille-Yosida Forma Geral, 9

de Lumer-Phillips, 10

das Três Linhas, 72

de Kato, 43

de Stone, 18

O Segundo Limite Fundamental, 8 